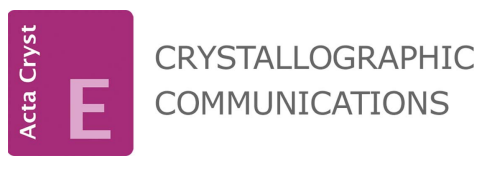

ISSN 2056-9890
Received 20 December 2021

Accepted 13 January 2022

Edited by B. Therrien, University of Neuchâtel, Switzerland

Keywords: crystal structure; molybdenum; salan ligand; cis-dioxo; $\mathrm{DODH}$.

CCDC references: $2142074 ; 2142073$

Supporting information: this article has supporting information at journals.iucr.org/e

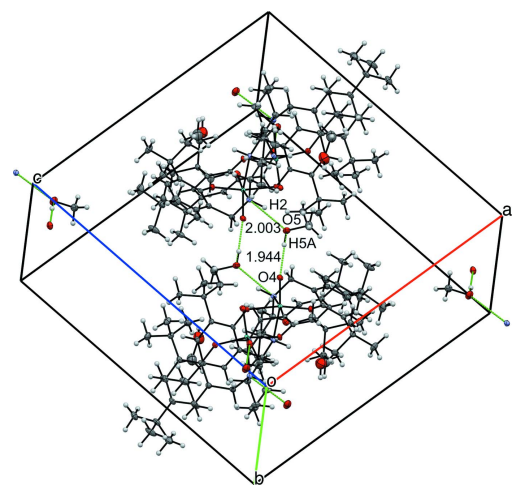

OPEN Ә ACCESS

\section{Crystal structures of two dioxomolybdenum complexes stabilized by salan ligands featuring phenyl and cyclohexyl backbones}

\author{
Tristhan Trieu-Tran, Stephenie N. Martinez, Jacob P. Brannon, S. Chantal E. Stieber \\ and Alex John*
}

Chemistry \& Biochemistry Department, California State Polytechnic University, Pomona, 3801 W. Temple Ave., Pomona,
CA 91768, USA. *Correspondence e-mail: ajohn@ @pp.edu

Two cis-dioxomolybdenum complexes based on salan ligands with different backbones are reported. The first complex, dioxido\{2,2'-[1,2-phenylenebis(iminomethylene)]bis(phenolato)\}molybdenum(VI) dimethylformamide disolvate, $\left[\mathrm{Mo}\left(\mathrm{C}_{20} \mathrm{H}_{18} \mathrm{~N}_{2} \mathrm{O}_{2}\right) \mathrm{O}_{2}\right] \cdot 2 \mathrm{C}_{3} \mathrm{H}_{7} \mathrm{NO}\left({ }^{\mathrm{Ph}} \mathrm{LMoO}_{2}, \mathbf{1 b}\right)$, features a phenyl backbone, while the second complex, $\left(6,6^{\prime}-\{[(\right.$ cyclohexane-1,2-diyl)bis(azanediyl)]bis(methylene)\}bis(2,4-di-tert-butylphenolato))dioxidomolybdenum(VI) methanol disolvate, $\left[\mathrm{Mo}\left(\mathrm{C}_{36} \mathrm{H}_{56} \mathrm{~N}_{2} \mathrm{O}_{2}\right) \mathrm{O}_{2}\right] \cdot 2 \mathrm{CH}_{3} \mathrm{OH}\left({ }^{\mathrm{Cy}} \mathrm{LMoO}_{2}, \mathbf{2 b}\right)$, is based on a cyclohexyl backbone. These complexes crystallized as solvated species, 1b.2DMF and 2b-2MeOH. The salan ligands ${ }^{\mathrm{Ph}} \mathrm{LH}_{2}$ (1a) and ${ }^{\mathrm{Cy}} \mathrm{LH}_{2}$ (2a) coordinate to the molybdenum center in these complexes $\mathbf{1 b}$ and $\mathbf{2 b}$ in a $\kappa^{2} N, \kappa^{2} O$ fashion, forming a distorted octahedral geometry. The $\mathrm{Mo}-\mathrm{N}$ and $\mathrm{Mo}-\mathrm{O}$ distances are $2.3475(16)$ and $1.9567(16) \AA$, respectively, in $\mathbf{1 b}$ while the corresponding measurements are $\mathrm{Mo}-\mathrm{N}=2.3412(12) \AA$, and $\mathrm{Mo}-\mathrm{O}=$ 1.9428 (10) $\AA$ for $\mathbf{2 b}$. A key geometrical feature is that the $\mathrm{N}-\mathrm{Mo}-\mathrm{N}$ angle of $72.40(4)^{\circ}$ in ${ }^{\mathrm{Cy}} \mathrm{LMoO}_{2}$ is slightly less than that of the ${ }^{\mathrm{Ph}} \mathrm{LMoO}_{2}$ angle of $75.18(6)^{\circ}$, which is attributed to the flexibility of the cyclohexane ring between the nitrogen as compared to the rigid phenyl ring in the ${ }^{\mathrm{Ph}} \mathrm{LMoO}_{2}$.

\section{Chemical context}

Molybdenum centers are present in the active sites of various enzymes including nitrogenases, sulfite oxidase, xanthine oxidase, and DMSO reductase that catalyze two-electron redox processes (Hille et al., 2014; Enemark et al., 2004; Hille, 1996). This is attributed to the large number of stable oxidation states and coordination environments that can be achieved, as well as the solubility of molybdate salts in water. A majority of these enzymes are referred to as oxo-molybdenum enzymes due to the presence of at least one $\mathrm{Mo}=\mathrm{O}$ moiety in the active site. The sulfite oxidase family of enzymes contains a cis-dioxo molybdenum(VI) $\left(L_{n} \mathrm{MoO}_{2}\right)$ center in its active site (Hille et al., 2014). Apart from being studied as models to understand biological systems, oxomolybdenum complexes have also found utility in processes such as olefin metathesis, olefin epoxidation, cytotoxic studies, and cyclic ester polymerizations (Hossain et al. 2020; Mayilmurugan et al. 2013; Yang et al. 2007). Mononuclear molybdenum complexes are generally distinguished by stretching frequencies $\{u(\mathrm{O}=\mathrm{Mo}=\mathrm{O})\}$ in the $910-950 \mathrm{~cm}^{-1}$ and $890-925 \mathrm{~cm}^{-1}$ regions, which are characteristic of a cis- $\mathrm{MoO}_{2}$ fragment (Chakravarthy \& Chand, 2011). A variety of ligand architectures have been successful in stabilizing the oxomolyb- 
denum core in these complexes (Ziegler et al. 2009; Subramanian et al. 1984; Rajan et al. 1983). Dioxomolybdenum complexes stabilized by salan ligands have been used extensively for various applications (Roy et al., 2017; Whiteoak et al., 2009). The modular nature for the synthesis of salan ligands allows for incorporation of steric and electronic variations in the ligand framework to tune the reactivity of the molybdenum center. We are exploring the utility of dioxomolybdenum complexes in catalyzing the deoxydehydration (DODH) reaction with a focus on understanding ligand effects on catalytic activity. This work reports synthesis and crystal structures of two molybdenum complexes including a crystallographically uncharacterized complex, dioxido $\left[2,2^{\prime}-\right.$ \{1,2-phenylenebis(iminomethylene)bis(phenolato)]molybdenum(VI), ${ }^{{ }^{P h}} \mathrm{LMoO}_{2}$ (1b) (Rajan et al. 1983). The second is a known complex with a new unit cell, (Ziegler et al., 2009), 6,6'$\{[($ cyclohexane-1,2-diyl)bis(azanediyl)]bis(methylene) $\}$ bis(2,4di-tert-butylphenolato))dioxidomolybdenum(VI), ${ }^{\mathrm{Cy}} \mathrm{LMoO}_{2}$ (2b).

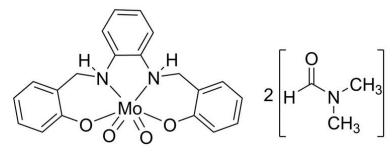

1b·2 DMF

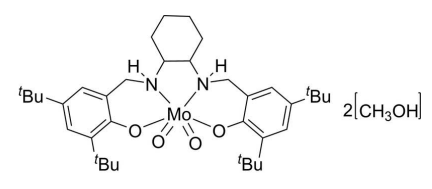

$2 \mathbf{b} \cdot 2 \mathrm{MeOH}$

\section{Structural commentary}

The asymmetric unit of ${ }^{\mathrm{Ph}} \mathrm{LMoO}_{2}(\mathbf{1 b})$ contains two molecules of ${ }^{\mathrm{Ph}} \mathrm{LMoO}_{2}$ and four molecules of dimethylformamide (DMF), as shown in Fig. 1. Fig. 2 shows one molecule of ${ }^{\mathrm{Ph}} \mathrm{LMoO}_{2}$ with hydrogen atoms and solvent removed for clarity. In this system, the salan ligand ${ }^{\mathrm{Ph}} \mathrm{LH}_{2}$ (1a) coordinates to the molybdenum center in a $\kappa^{2} N, \kappa^{2} O$ fashion, forming a distorted octahedral geometry. The angles formed around the molybdenum core are $80.23(6)^{\circ}$ for $\mathrm{O} 1-\mathrm{Mo} 01-\mathrm{N} 1$, $157.78(6)^{\circ}$ for $\mathrm{O} 1-\mathrm{Mo} 01-\mathrm{O} 2,75.18(6)^{\circ}$ for $\mathrm{N} 1-\mathrm{Mo} 01-$ $\mathrm{N} 2$, and $109.80(7)^{\circ}$ for $\mathrm{O} 3-\mathrm{Mo} 01-\mathrm{O} 4$. These angles are consistent with a system that is significantly distorted from octahedral geometry with bond angles resulting from the salan ligand ranging from $75.18(6)$ to $84.38(7)^{\circ}$, while the angle between the 'oxo' oxygens of $109.80(7)^{\circ}$ is close to the ideal tetrahedral angle of $109.5^{\circ}$. Analogous bond angles in the second molecule in the unit cell are the same within $0.01 \AA$. The bond distances between the molybdenum center and ligand atoms for Mo01-N1 and Mo01-O1 are 2.3475 (16) and 1.9567 (16) $\AA$, respectively. The notable bond distances from the salan ligand are $\mathrm{O} 1-\mathrm{C} 1$ at 1.377 (2) $\AA, \mathrm{N} 1-\mathrm{C} 7$ at 1.486 (3) $⿱ \mathrm{~A}, \mathrm{C} 2-\mathrm{C} 7$ at 1.515 (3) $\mathrm{A}, \mathrm{N} 1-\mathrm{C} 8$ at 1.389 (8) $\AA$, and $\mathrm{C} 8-\mathrm{C} 13$ at 1.419 (3) $\AA$. Analogous bond distances in the second molecule in the unit cell are the same within $0.01 \AA$ as distances for $\mathrm{O} 1-\mathrm{C} 1$ and $\mathrm{N} 1-\mathrm{C} 8$, respectively. The other bond distances have variations of $0.2-0.3 \AA$, with $\mathrm{N} 3-\mathrm{C} 27$ at 1.519 (3) $\AA, \mathrm{C} 26-\mathrm{C} 27$ at 1.490 (3) $\AA$, and $\mathrm{C} 28-\mathrm{C} 33$ at $1.392(3) \AA$.

The asymmetric unit of ${ }^{\mathrm{Cy}} \mathrm{LMoO}_{2}(\mathbf{2 b})$ contains one molecule of ${ }^{\mathrm{Cy}} \mathrm{LMoO}_{2}$ and two molecules of methanol (MeOH)

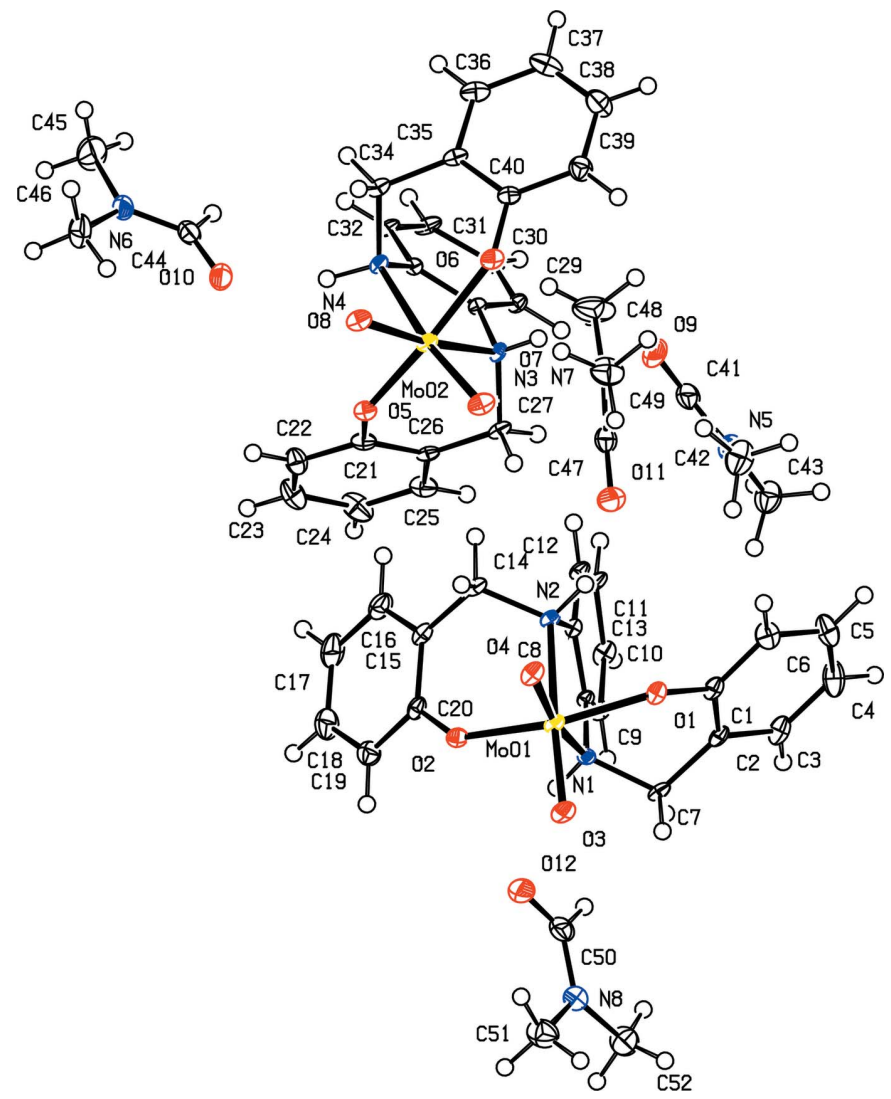

Figure 1

View of $2\left[{ }^{\mathrm{Ph}} \mathrm{LMoO}_{2}\right] \cdot 4[\mathrm{DMF}]$ (1b) with $50 \%$ probability ellipsoids.

(Fig. 3). The salan ligand ${ }^{\mathrm{Cy}} \mathrm{LH}_{2}$ (2a) binds in the same $\kappa^{2} N, \kappa^{2} O$ fashion that complex $\mathbf{1 b}$ does. Fig. 4 shows ${ }^{\mathrm{Cy}} \mathrm{LMoO}_{2}$ with the hydrogen atoms removed for clarity. The complex also has a distorted octahedral geometry with angles of O3$\mathrm{Mo} 01-\mathrm{O} 1$ at $96.36(5)^{\circ}, \mathrm{O} 1-\mathrm{Mo} 01-\mathrm{N} 1$ at $76.73(4)^{\circ}, \mathrm{N} 1-$ $\mathrm{Mo} 01-\mathrm{N} 2$ at $72.40(4)^{\circ}, \mathrm{N} 2-\mathrm{Mo} 01-\mathrm{O} 2$ at $78.91(4)^{\circ}, \mathrm{O} 2-$ $\mathrm{Mo} 01-\mathrm{O} 4$ at $100.19(5)^{\circ}, \mathrm{O} 2-\mathrm{Mo} 01-\mathrm{O} 3$ at $94.58(5)^{\circ}$. These

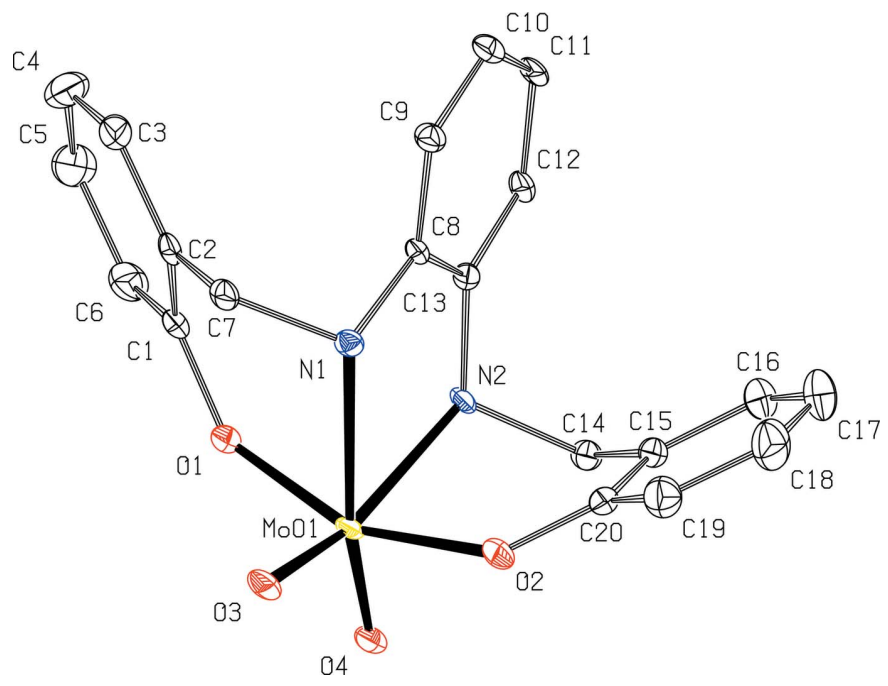

Figure 2

View of one molecule of ${ }^{\mathrm{Ph}} \mathrm{LMoO}_{2}(\mathbf{1 b})$ with $50 \%$ probability ellipsoids. The DMF molecule and $\mathrm{H}$ atoms are omitted for clarity. 


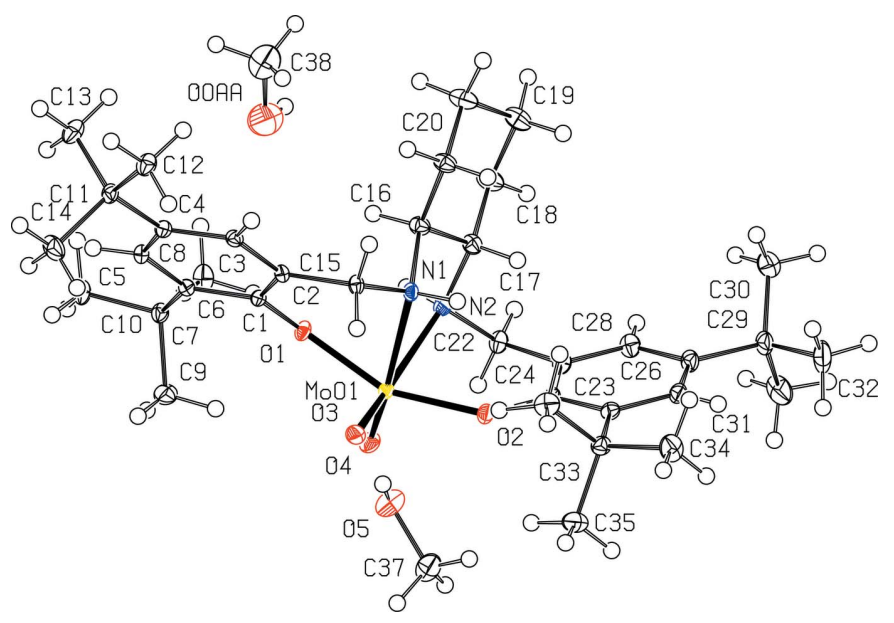

Figure 3

View of one molecule of ${ }^{\mathrm{cy}} \mathrm{LMoO}_{2} \cdot 2 \mathrm{MeOH} \quad(\mathbf{2 b})$ with $50 \%$ probability ellipsoids.

angles are between 5 and $10^{\circ}$ of the ideal $90^{\circ}$ for octahedral geometry. The $\mathrm{N} 1-\mathrm{Mo} 01-\mathrm{N} 2$ angle at $72.40(4)^{\circ}$ is slightly less than that of the ${ }^{\mathrm{Ph}} \mathrm{LMoO}_{2}$ angle of $75.81(6)^{\circ}$, which is attributed to the flexibility of the cyclohexane ring between the nitrogen atoms compared to the rigid phenyl ring in the ${ }^{\mathrm{Ph}} \mathrm{LMoO}_{2}$. Metal-ligand bond distances are found for Mo01-

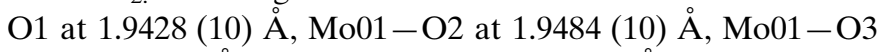
at 1.7125 (10) $\mathrm{A}, \mathrm{Mo0} 1-\mathrm{O} 4$ at 1.7226 (11) $\AA$, Mo01-N1 at

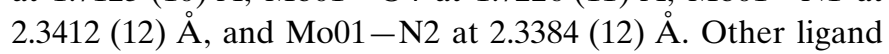
distances and bond lengths within the phenyl rings are consistent with analagous distances in ${ }^{\mathrm{Ph}} \mathrm{LMoO}_{2}$ (1b). The cylohexane bond distances are consistent with single $\mathrm{C}-\mathrm{C}$ bonds. The bond lengths observed are not statistically different than those reported by Ziegler et al. (2009). There are a few statistically different angles, specifically around the molybdenum center where Table 1 shows the correlating bond angles. These bond-angle differences are most likely due to improved $R 1$ of $2.78 \%$ as compared to the previously reported

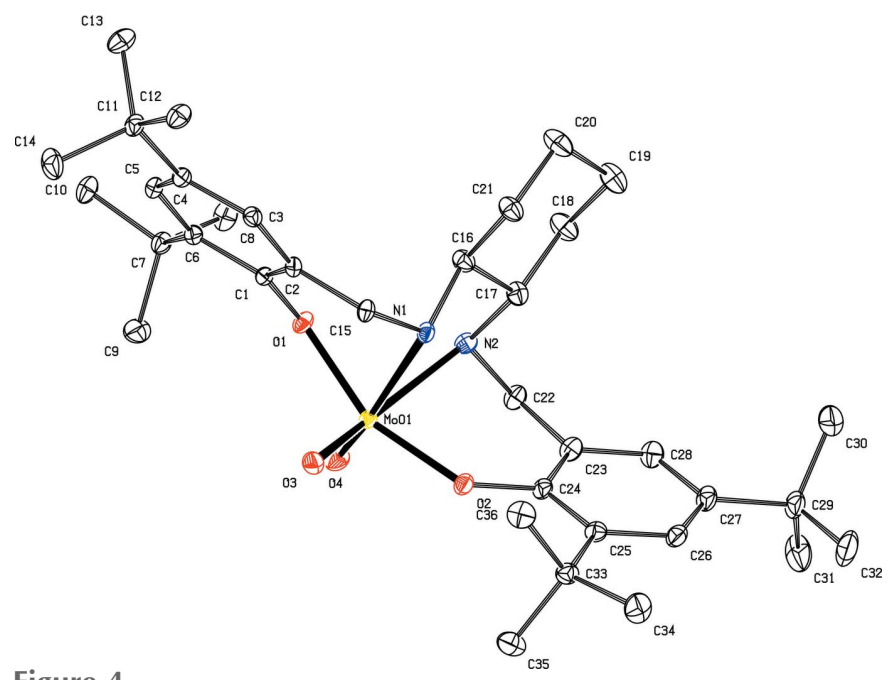

Figure 4

View of one molecule of ${ }^{\text {cy }} \mathrm{LMoO}_{2}$ (2b) with $50 \%$ probability ellipsoids. The $\mathrm{MeOH}$ molecules and $\mathrm{H}$ atoms are omitted for clarity.
Table 1

Comparison of bond angles $\left({ }^{\circ}\right)$ between ${ }^{\mathrm{Cy}} \mathrm{LMoO}_{2}(\mathbf{2 b})$ with $R 1$ of $2.78 \%$ and reported structure from Ziegler et al. (2009) with $R 1$ of $5.5 \%$.

\begin{tabular}{llll}
\hline $\mathbf{2 b}$ & Angle & Reported $^{a}$ & Angle \\
\hline O4-Mo01-O2 & $100.19(5)$ & O2-Mo1-O62 & $94.3(2)$ \\
O2-Mo01-N2 & $78.91(4)$ & O62-Mo1-N2 & $86.4(2)$ \\
N1-Mo01-N2 & $72.40(4)$ & N5-Mo1-N2 & $72.0(2)$ \\
O1-Mo01-N1 & $76.73(4)$ & N5-Mo1-O12 & $82.7(2)$ \\
O3-Mo01-O1 & $96.36(5)$ & O12-Mo1-O1 & $93.8(2)$ \\
O3-Mo01-O4 & $108.55(5)$ & O2-Mo1-O1 & $107.6(2)$ \\
\hline
\end{tabular}

Note: (a) Ziegler et al. (2009).

$R 1$ of $5.5 \%$ and higher solvent disorder in the reported structure.

\section{Supramolecular features}

${ }^{\mathrm{Ph}} \mathrm{LMoO}_{2}$ (1) : A single molecule of ${ }^{\mathrm{Ph}} \mathrm{LMoO}_{2}$ is hydrogen bonded to one disordered DMF molecule, as shown in Fig. 5, with a distance of $2.03 \AA$ for O11 ...H008 (Table 2). A second hydrogen bond interaction is between $\mathrm{O} 9-\mathrm{H} 00 D$ with a distance of $2.16(3) \AA$. Corresponding hydrogen bond distances in the second molecule in the unit cell are similar. There are three formula units within the contents of the unit cell. Perpendicular $\pi$-stacking between ${ }^{\mathrm{Ph}} \mathrm{LMoO}_{2}$ molecules is observed between $\mathrm{C} 5$ and the aryl ring centroid (C35-C39) with a distance of $4.597 \AA$.

${ }^{\mathrm{Cy}} \mathrm{LMoO}_{2}$ (2) $)$ : There are four molecules of ${ }^{\mathrm{Cy}} \mathrm{LMoO}_{2}$ in the unit cell of this system and the complex is stabilized via hydrogen bonding to the solvent $\mathrm{MeOH}$ molecule (1.94 $\AA$ for O4 . .H5 $A$ and $2.00 \AA$ for O5 . H 2 ; Table 3), as seen in Fig. 6 . There is no indication that there are $\pi$-stacking interactions between the two molecules. In comparing the hydrogen

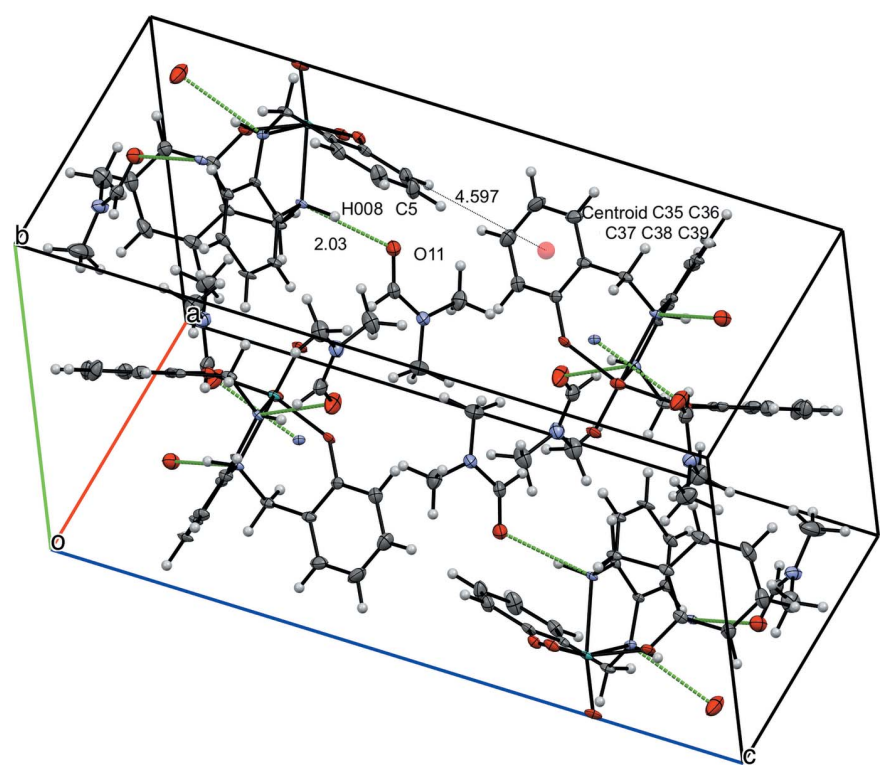

Figure 5

View of six molecules of ${ }^{\mathrm{Ph}} \mathrm{LMoO}_{2}$ and five molecules of DMF in the unit cell with $50 \%$ probability ellipsoids, highlighting intermolecular distances. Distances between $\mathrm{H}$ atoms are listed without standard deviations because the $\mathrm{H}$ atoms were positionally fixed.. 
Table 2

Hydrogen-bond geometry $\left(\AA,^{\circ}\right)$ for $\mathbf{1 b}$.

\begin{tabular}{|c|c|c|c|c|}
\hline$D-\mathrm{H} \cdots A$ & $D-\mathrm{H}$ & $\mathrm{H} \cdots A$ & $D \cdots A$ & $D-\mathrm{H} \cdots A$ \\
\hline $\mathrm{N} 2-\mathrm{H} 008 \cdots \mathrm{O} 11$ & 1.00 & 2.03 & $2.958(2)$ & 154 \\
\hline $\mathrm{N} 4-\mathrm{H} 009 \cdots \mathrm{O} 10$ & 1.00 & 1.99 & $2.924(3)$ & 154 \\
\hline $\mathrm{N} 1-\mathrm{H} 00 D \cdots \mathrm{O} 12$ & $0.85(3)$ & $2.15(3)$ & $2.949(3)$ & $157(2)$ \\
\hline 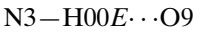 & $0.79(3)$ & $2.16(3)$ & $2.885(3)$ & $154(3)$ \\
\hline
\end{tabular}

Table 3

Hydrogen-bond geometry $\left(\AA{ }^{\circ}\right)$ for $\mathbf{2 b}$.

\begin{tabular}{lllll}
\hline$D-\mathrm{H} \cdots A$ & $D-\mathrm{H}$ & $\mathrm{H} \cdots A$ & $D \cdots A$ & $D-\mathrm{H} \cdots A$ \\
\hline $\mathrm{N} 2-\mathrm{H} 2 \cdots \mathrm{O} 5^{\mathrm{i}}$ & 1.00 & 2.00 & $2.9319(16)$ & 153 \\
$\mathrm{O} 5-\mathrm{H} 5 A \cdots \mathrm{O} 4$ & 0.84 & 1.94 & $2.7837(16)$ & 177 \\
\hline
\end{tabular}

Symmetry code: (i) $-x+1,-y+1,-z+1$.

bonding with the previously reported structure, the main difference is the formation of hydrogen-bonded tetramers containing two molecules of $\mathbf{2 b}$ and two molecules of methanol in the current structure. The previously reported structure had one resolved molecule of methanol and one disordered oxygen atom, which form a hydrogen-bonded trimer with one molecule of ${ }^{\mathrm{Cy}} \mathrm{LMoO}_{2}$ (Ziegler et al., 2009).

\section{Database survey}

A database search of the Cambridge Structural Database (CSD; Groom et al., 2016) (webCSD accessed September 22, 2021) and SciFinder (SciFinder, 2021) did not yield any exact matches to the crystal structure for ${ }^{\mathrm{Ph}} \mathrm{LMoO}_{2}(\mathbf{1 b})$. There was a similar crystal structure found with the imine form of the

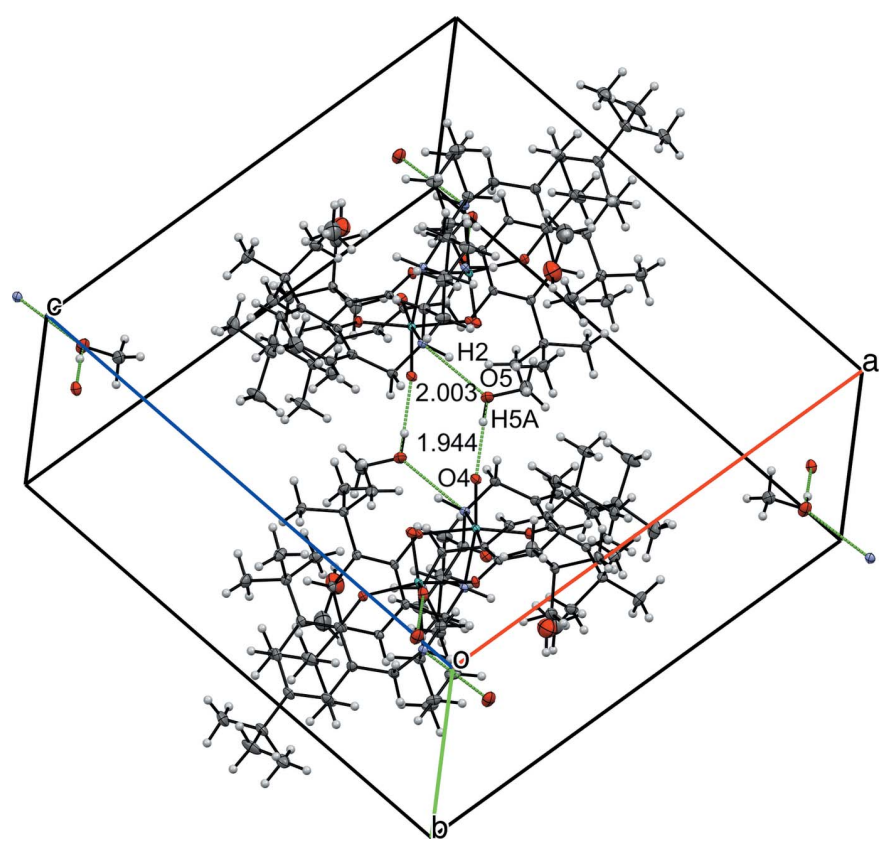

Figure 6

View of four molecules of ${ }^{\text {cy }} \mathrm{LMoO}_{2}$ and six molecules of methanol in the unit cell with $50 \%$ probability ellipsoids, highlighting intermolecular distances. Distances between $\mathrm{H}$ atoms are listed without standard deviations because the $\mathrm{H}$ atoms were positionally fixed. ligand (Salen) $\mathrm{MoO}_{2}$. A search for ${ }^{\mathrm{Cy}} \mathrm{LMoO}_{2}$ (2b) in the CSD (webCSD accessed September 22, 2021) shows that there is a known structure of the molecule with a different unit cell with accession code HUWGOW (Ziegler et al., 2009). The SciFinder search resulted in the same sources being found. The current structure for ${ }^{\mathrm{Cy}} \mathrm{LMoO}_{2}(\mathbf{2 b})$ was solved in space group $P 2_{1} / n$ compared with $P 3_{1}$ for HUWGOW. The primary additional differences in the structures is an improved $R 1$ of $2.78 \%$ and more clearly resolved methanol solvent, as compared to the previously reported $R 1$ of $5.5 \%$ and more disordered methanol solvent (Ziegler et al., 2009).

\section{Synthesis and crystallization}

The salan ligands used for stabilizing $\left[\mathrm{MoO}_{2}\right]^{2+}$ in the complexes ${ }^{\mathrm{Ph}} \mathrm{LMoO}_{2}$ (1b) (Rajan et al. 1983) and ${ }^{\mathrm{Cy}} \mathrm{LMoO}_{2}$ (2b) (Ziegler et al., 2009) were synthesized by the reductive amination of the corresponding salicylaldehyde and diamine. The ligands ${ }^{\mathrm{Ph}} \mathrm{LH}_{2}$ (1a) and ${ }^{\mathrm{Cy}} \mathrm{LH}_{2}$ (2a) were synthesized as off-white solids in $86 \%$ and $58 \%$ yields, respectively. The reaction scheme is shown in Fig. 7. Both ligands were successfully characterized by NMR and IR spectroscopy. A salient feature in the ${ }^{1} \mathrm{H}$ NMR spectra of both ligands as compared to the precursor salen compounds was the disappearance of the aldimine peak $(\sim 8.50 \mathrm{ppm})$ and the appearance of the benzylic resonances $\sim 4.00 \mathrm{ppm}$. The molybdenum complexes ${ }^{\mathrm{Ph}} \mathrm{LMoO}_{2}(\mathbf{1 b})$ and ${ }^{\mathrm{Cy}} \mathrm{LMoO}_{2}$ (2b) were synthesized in $86 \%$ and $42 \%$ yields, respectively, by the reaction of the corresponding ligands with $\mathrm{MoO}_{2}(\mathrm{acac})_{2}$ in methanol or acetonitrile as solvent. Complexes $\mathbf{1 b}$ and $\mathbf{2 b}$ were also characterized by NMR and IR spectroscopy. Both complexes exhibited stretches $\left\{\left[(\mathrm{Mo}=\mathrm{O})=916\right.\right.$ and $876 \mathrm{~cm}^{-1}(\mathbf{1 b}) ; 903$ and $\left.875 \mathrm{~cm}^{-1}(\mathbf{2 b})\right]$ characteristic of a cis-dioxo molybdenum core in the IR spectrum.

\section{Procedure for synthesis of ligands}

${ }^{\mathrm{Ph}} \mathrm{LH}_{2}$ (1a): To a solution of 1,2-phenylenediamine $(0.764 \mathrm{~g}$, $7.20 \mathrm{mmol}$ ) in methanol ( $\mathrm{ca} 7 \mathrm{ml}$ ) was added a solution of salicylaldehyde $(1.76 \mathrm{ml}, 14.9 \mathrm{mmol})$ in methanol (ca $8 \mathrm{ml})$. The mixture was stirred for $6 \mathrm{~h}$ at room temperature. The orange precipitate that formed during this period was filtered and washed with methanol, then dried under high vacuum to yield the salophen product as an orange solid $(2.19 \mathrm{~g}, 98 \%) .{ }^{1} \mathrm{H}$ NMR $\left(\mathrm{CDCl}_{3}, 400 \mathrm{MHz}, 300 \mathrm{~K}\right) \delta 13.0(s, 2 \mathrm{H}), 8.63(s, 2 \mathrm{H})$, $7.38\left(d,{ }^{3} J_{\mathrm{H} H}=8 \mathrm{~Hz}, 2 \mathrm{H}\right), 7.35-7.33(m, 2 \mathrm{H}), 7.26-7.22(m$, $2 \mathrm{H}), 7.05\left(d,{ }^{3} J_{\mathrm{H} H}=8 \mathrm{~Hz}, 2 \mathrm{H}\right), 6.92\left(t,{ }^{3} J_{\mathrm{HH}}=8 \mathrm{~Hz}, 2 \mathrm{H}\right)$.

To a mixture of methanol $(c a .8 \mathrm{ml})$ and diethyl ether ( $c$ a $8 \mathrm{ml}$ ), was added salophen $(1.52 \mathrm{~g}, 4.81 \mathrm{mmol}$ ) followed by $\mathrm{NaBH}_{4}(1.67 \mathrm{~g}, 44.4 \mathrm{mmol})$, and the reaction mixture was stirred at room temperature for $1 \mathrm{~h}$. When the yellow color of the solution changed to colorless, it was transferred into a separatory funnel and DI $\mathrm{H}_{2} \mathrm{O}(\mathrm{ca} 15 \mathrm{ml})$ was added followed by ethyl acetate $(2 \times c a 15 \mathrm{ml})$ for extraction. The organic solution was separated and combined, then washed with saturated $\mathrm{NaCl}$ solution ( $c$ a $20 \mathrm{ml}$ ). The organic layer was dried over anhydrous $\mathrm{Na}_{2} \mathrm{SO}_{4}$ and filtered. The filtrate was concentrated under vacuum to give a light-yellow solid, which was dried under high vacuum. The color of the solid changed 

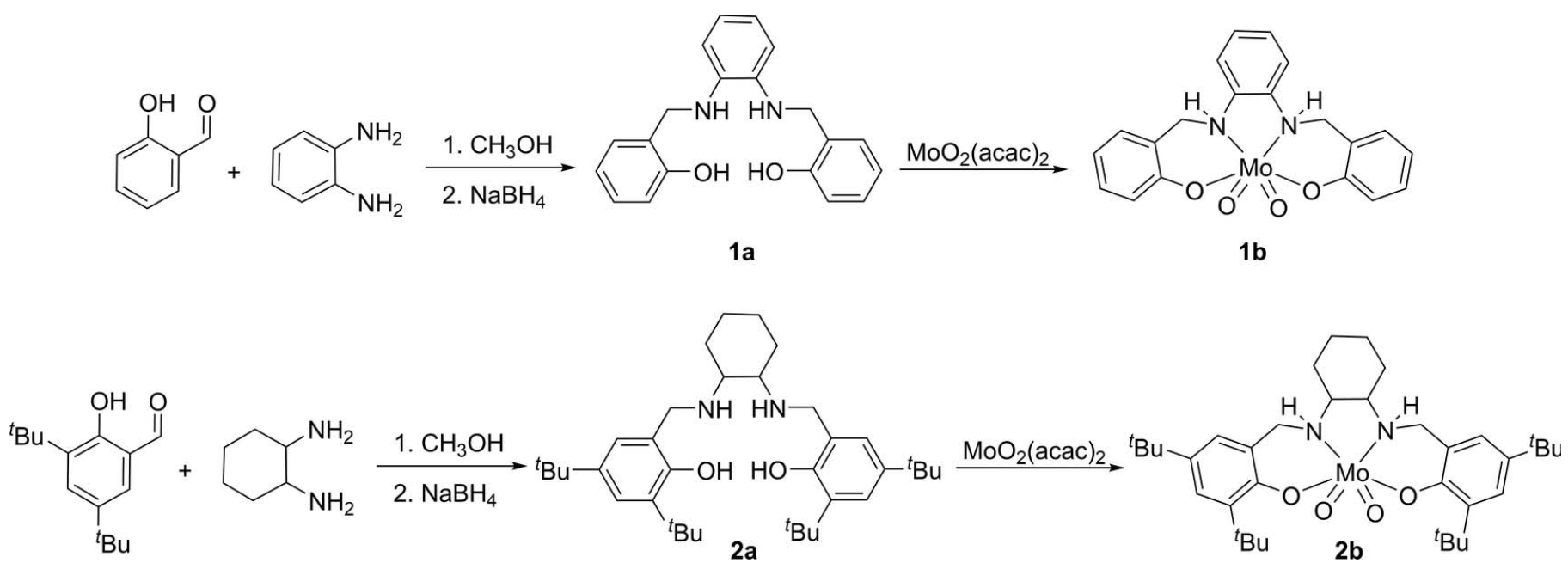

Figure 7

Synthesis of the dioxomolybdenum complexes $\mathbf{1 b}$ and $\mathbf{2 b}$.

to light brown after $2 \mathrm{~h}$ under high vacuum to yield the product $(1.32 \mathrm{~g}, 86 \%) .{ }^{1} \mathrm{H}$ NMR $\left(\mathrm{CDCl}_{3}, 400 \mathrm{MHz}, 301 \mathrm{~K}\right) \delta$ 7.24-7.19 (m, 4H), 6.96-6.94 ( $m, 4 \mathrm{H}), 6.89\left(t,{ }^{3} J_{\mathrm{H} H}=8 \mathrm{~Hz}, 2 \mathrm{H}\right)$, $6.86\left(t,{ }^{3} J_{\mathrm{H} H}=8 \mathrm{~Hz}, 2 \mathrm{H}\right), 4.40(s, 4 \mathrm{H})$.

${ }^{\mathrm{Cy}} \mathrm{LH}_{2}$ (2a): A $100 \mathrm{~mL}$ round-bottom flask was charged with trans-1,2-diaminocyclohexane $(0.448 \mathrm{~g}, 4.38 \mathrm{mmol})$, methanol (ca. $16 \mathrm{~mL})$, and 3,5-di-tert-butylsalicylaldehyde $(2.05 \mathrm{~g}$, $17.5 \mathrm{mmol})$. The solution was stirred for $24 \mathrm{~h}$ at room temperature. The solution resulted in a bright-yellow precipitate. The precipitate was then collected by gravity filtration and washed with cold methanol. The precipitate was dried under high vacuum to remove any residual solvent and yield the salen product $(3.85 \mathrm{~g}, 81 \%) .{ }^{1} \mathrm{H} \mathrm{NMR}\left(\mathrm{CDCl}_{3}, 400 \mathrm{MHz}\right.$, $301 \mathrm{~K}) \delta 13.6(b r, 2 \mathrm{H}), 8.33(s, 2 \mathrm{H}), 7.34(s, 2 \mathrm{H}), 7.02(s, 2 \mathrm{H})$, $3.37(b r, 2 \mathrm{H}), 1.98-1.77(m, 4 \mathrm{H}), 1.40(s, 18 \mathrm{H}), 1.33-1.29(m$, $4 \mathrm{H}), 1.24(s, 18 \mathrm{H})$.

A $100 \mathrm{~mL}$ round-bottom flask was charged with the salen product $(1.00 \mathrm{~g}, 2.00 \mathrm{mmol})$, methanol $(c a 3 \mathrm{~mL})$, and THF (ca $25 \mathrm{~mL}$ ). $\mathrm{NaBH}_{4}$ (9 equivalents) was slowly added into the reaction mixture until the solution was colorless. The reaction was quenched with DI water ( $c$ a $20 \mathrm{~mL}$ ), and the product was extracted with ethyl acetate $(2 \times c a 10 \mathrm{ml})$ using a separatory funnel. The combined organic layers were dried using anhydrous $\mathrm{Na}_{2} \mathrm{SO}_{4}$ and was concentrated under vacuum using the rotary evaporator. The product was then put under high vacuum overnight to ensure it was completely dry $(0.577 \mathrm{~g}$, $58 \%) .{ }^{1} \mathrm{H}$ NMR $\left(\mathrm{CDCl}_{3}, 400 \mathrm{MHz}, 301 \mathrm{~K}\right) \delta 7.22\left(d,{ }^{4} J_{\mathrm{H} H}=\right.$ $4 \mathrm{~Hz}, 2 \mathrm{H}), 6.87\left(d,{ }^{4} J_{\mathrm{H} H}=4 \mathrm{~Hz}, 2 \mathrm{H}\right), 4.05\left(d,{ }^{2} J_{\mathrm{H} H}=16 \mathrm{~Hz}\right.$, $2 \mathrm{H}), 3.90\left(d,{ }^{2} J_{\mathrm{H} H}=16 \mathrm{~Hz}, 2 \mathrm{H}\right), 2.51(b r, 2 \mathrm{H}), 2.19(b r, 2 \mathrm{H})$, $1.72(b r, 2 \mathrm{H}), 1.44-1.41(\mathrm{~m}, 2 \mathrm{H}), 1.38(s, 18 \mathrm{H}), 1.28(s, 18 \mathrm{H})$, 1.23-1.20 ( $m, 4 \mathrm{H})$.

\section{Procedure for synthesis of molybdenum complexes}

Dioxido[2,2' $-\{1,2$-phenylenebis(iminomethylene) $\}$ bis(phenolato)]molybdenum(VI) ( $\left.{ }^{\mathrm{Ph}} \mathrm{LMoO}_{2}, \mathbf{1 b}\right)$ : To a solution of $\mathbf{1 a}$ $(1.04 \mathrm{~g}, 3.29 \mathrm{mmol})$ in acetonitrile $(c a 20 \mathrm{ml})$ was added $\mathrm{MoO}_{2}(\mathrm{acac})_{2}(1.07 \mathrm{~g}, 3.30 \mathrm{mmol})$ and the mixture was stirred at room temperature for $10 \mathrm{~min}$. The yellow precipitate that formed was filtered and then dried under vacuum to yield the complex as yellow solid $(1.24 \mathrm{~g}, 86 \%) .{ }^{1} \mathrm{H}$ NMR (DMSO- $d_{6}$, $400 \mathrm{MHz}, 301 \mathrm{~K}) \delta 7.55\left(d,{ }^{3} J_{\mathrm{H} H}=8 \mathrm{~Hz}, 1 \mathrm{H}\right), 7.37-7.35(\mathrm{~m}$, $1 \mathrm{H}), 7.19-7.10(m, 4 \mathrm{H}), 7.07-7.05(m, 1 \mathrm{H}), 7.02-6.98(m, 2 \mathrm{H})$, $6.91\left(d,{ }^{3} J_{\mathrm{H} H}=8 \mathrm{~Hz}, 1 \mathrm{H}\right), 6.85-6.83(m, 1 \mathrm{H}), 6.80\left(d,{ }^{3} J_{\mathrm{H} H}=\right.$ $8 \mathrm{~Hz}, 1 \mathrm{H}), 6.76-6.68(m, 2 \mathrm{H}), 6.63\left(d,{ }^{3} J_{\mathrm{HH}}=8 \mathrm{~Hz}, 1 \mathrm{H}\right), 6.59$ $\left(d,{ }^{3} J_{\mathrm{H} H}=8 \mathrm{~Hz}, 1 \mathrm{H}\right), 6.42\left(d,{ }^{2} J_{\mathrm{H} H}=12 \mathrm{~Hz}, 1 \mathrm{H}\right), 5.24\left(d,{ }^{2} J_{\mathrm{H} H}=\right.$ $16 \mathrm{~Hz}, 1 \mathrm{H}), 5.16\left(d,{ }^{2} J_{\mathrm{H} H}=16 \mathrm{~Hz}, 1 \mathrm{H}\right), 4.94\left(d,{ }^{2} J_{\mathrm{H} H}=16 \mathrm{~Hz}\right.$, $1 \mathrm{H}), 4.20\left(d,{ }^{2} J_{\mathrm{H} H}=12 \mathrm{~Hz}, 1 \mathrm{H}\right) .{ }^{13} \mathrm{C}\left\{{ }^{1} \mathrm{H}\right\}$ NMR (DMSO- $d_{6}$, $100 \mathrm{MHz}, 301 \mathrm{~K}) \delta 163.0,160.2,155.6,148.0,141.1,130.5$, 129.1, 129.0, 128.9, 128.0, 127.9, 125.9, 124.3, 122.9, 120.1, 119.2, 119.1, 118.9, 117.8, 115.3, 111.1, 53.7, 53.6. Selected IR $\left(\mathrm{cm}^{-1}\right)$ : $3127 v\left(2^{\circ} \mathrm{N}-\mathrm{H}\right) ; 916,876 v(\mathrm{Mo}=\mathrm{O})$.

Crystals of ${ }^{\mathrm{Ph}} \mathrm{LMoO}_{2}$, $\mathbf{1 b}$ were grown by forming a supersaturated solution of the complex in DMF and layering with hexanes. The solution was placed in a refrigerator at $268 \mathrm{~K}$ for 1.5 months. Orange-yellow crystals were observed to grow and were collected for structural determination.

$\left(6,6^{\prime}-\{[(\right.$ Cyclohexane-1,2-diyl)bis(azanediyl)]bis(methylene)\}bis(2,4-di-tert-butylphenolato))dioxidomolybdenum(VI) $\left({ }^{\mathrm{Cy}} \mathrm{LMoO}_{2}, \mathbf{2 b}\right)$ : A round-bottom flask equipped with a magnetic stirring bar was charged with $\mathrm{MoO}_{2}(\mathrm{acac})_{2}(0.165 \mathrm{~g}$, $0.506 \mathrm{mmol})$ and methanol $(c a .10 \mathrm{~mL})$. The solution was stirred, and $2 \mathrm{a}(0.27 \mathrm{~g}, 0.51 \mathrm{mmol})$ was added to the $\mathrm{MoO}_{2}(\mathrm{acac})_{2}$ dissolved in methanol. The solution was stirred overnight when it turned orange. The solution was filtered, and the solvent removed by evaporation under vacuum to obtain an orange precipitate. The precipitate was triturated with methanol, producing an orange solid, which was separated by gravity filtration and was washed twice with cold methanol $(0.108 \mathrm{~g}, 42 \%) .{ }^{1} \mathrm{H}$ NMR $\left(\mathrm{CDCl}_{3}, 400 \mathrm{MHz}, 301 \mathrm{~K}\right)$ $\delta 7.26(s, 2 \mathrm{H}), 6.86(s, 2 \mathrm{H}), 5.28\left(d,{ }^{2} J_{\mathrm{HH}}=16 \mathrm{~Hz}, 2 \mathrm{H}\right), 4.18(d$, $\left.{ }^{2} J_{\mathrm{H} H}=12 \mathrm{~Hz}, 2 \mathrm{H}\right), 2.34-2.28(m, 4 \mathrm{H}), 1.43(s, 18 \mathrm{H}), 1.30(s$, $18 \mathrm{H}), 1.19-1.17(m, 4 \mathrm{H}), 0.88-0.85(m, 4 \mathrm{H}) .{ }^{13} \mathrm{C}\left\{{ }^{1} \mathrm{H}\right\}$ NMR $\left(\mathrm{CDCl}_{3}, 100 \mathrm{MHz}, 301 \mathrm{~K}\right) \delta 157.1,152.1,142.8,142.3,142.0$, 138.0, 137.7, 137.6, 125.7, 125.4, 124.1, 124.0, 123.0, 122.9, 120.0, $119.6,65.19,58.9,57.6,53.4,50.9,50.5,35.2,35.1,34.3,34.2$, 
Table 4

Experimental details.

1b

Crystal data

Chemical formula

$M_{\mathrm{r}}$

Crystal system, space group

Temperature (K)

$a, b, c(\AA)$

$\alpha, \beta, \gamma\left({ }^{\circ}\right)$

$V\left(\AA^{3}\right)$

Z

Radiation type

$\mu\left(\mathrm{mm}^{-1}\right)$

Crystal size (mm)

Data collection

Diffractometer

Absorption correction

$T_{\min }, T_{\max }$

No. of measured, independent and

observed $[I>2 \sigma(I)]$ reflections

$R_{\text {int }}$

$(\sin \theta / \lambda)_{\max }\left(\AA^{-1}\right)$

Refinement

$R\left[F^{2}>2 \sigma\left(F^{2}\right)\right], w R\left(F^{2}\right), S$

No. of reflections

No. of parameters

$\mathrm{H}$-atom treatment

$\Delta \rho_{\max }, \Delta \rho_{\min }\left(\mathrm{e} \AA^{-3}\right)$
$\left[\mathrm{Mo}\left(\mathrm{C}_{20} \mathrm{H}_{18} \mathrm{~N}_{2} \mathrm{O}_{2}\right) \mathrm{O}_{2}\right] \cdot 2 \mathrm{C}_{3} \mathrm{H}_{7} \mathrm{NO}$

592.49

Triclinic, $P \overline{1}$

100

$9.601,12.860,21.428$

$91.44,91.49,93.22$

2639.8

4

Mo $K \alpha$

0.54

$0.34 \times 0.29 \times 0.29$

Bruker APEXII CCD

Multi-scan (SADABS; Bruker, 2016)

$0.664,0.737$

$146655,7625,6364$

0.056

0.641

$0.035,0.065,1.06$

7625

683

$\mathrm{H}$ atoms treated by a mixture of independent and constrained refinement

$0.35,-0.38$ 2b

$\left[\mathrm{Mo}\left(\mathrm{C}_{36} \mathrm{H}_{56} \mathrm{~N}_{2} \mathrm{O}_{2}\right) \mathrm{O}_{2}\right] \cdot 2 \mathrm{CH}_{4} \mathrm{O}$

740.84

Monoclinic, $P 2_{1} / n$

105

18.4889 (14), 10.9722 (8), 19.1517 (14)

$90,94.035(2), 90$

$3875.6(5)$

4

Mo $K \alpha$

0.38

$0.2 \times 0.18 \times 0.1$

Bruker APEXII CCD

Multi-scan (SADABS; Bruker, 2016) $0.672,0.750$

$29075,9532,8724$

0.026

0.667

$0.028,0.070,1.07$

9532

440

H-atom parameters constrained

$0.52,-0.52$

Computer programs: APEX2 and SAINT (Bruker, 2016), SHELXT (Sheldrick, 2015), SHELXL (Sheldrick, 2008), and OLEX2 (Dolomanov et al., 2009).

33.0, 31.6, 31.6, 31.5, 29.9, 29.9, 28.9, 24.5, 24.3, 24.1. Selected

IR $\left(\mathrm{cm}^{-1}\right)$ : $903,875 v(\mathrm{Mo}=\mathrm{O})$.

Crystals of ${ }^{\mathrm{Cy}} \mathrm{LMoO}_{2}$, $2 \mathbf{b}$ were grown by using a supersaturated solution of the complex dissolved in methanol and allowed to undergo slow evaporation over $2 \mathrm{~d}$. A similar vial was also refrigerated where crystals were seen to form as well. The crystals from the slow evaporation set up were cropped and the orange-yellow crystals were used for structure determination.

\section{Refinement}

Crystal data, data collection, and refinement details are listed in Table 4. Hydrogen atoms were placed at ideal positions with $\mathrm{C}-\mathrm{H}$ distances at 0.95 for $\mathrm{CH}$ and $0.99 \AA$ for $s p^{3} \mathrm{CH}_{2}$ and $\mathrm{CH}_{3}$ using HFIX commands, and refined using a riding model with $U_{\text {iso }}(\mathrm{H})=1.2 U_{\text {eq }}(\mathrm{C})$ for $\mathrm{CH}, \mathrm{CH}_{2}$, and $\mathrm{CH}_{3}$. The structure for ${ }^{\mathrm{Ph}} \mathrm{MoO}_{2}$ (1) $)$ was initially refined in the trigonal crystal system $P 3_{2} 21$; however, this resulted in the solvent DMF having a high level of disorder with many checkCIF errors.

\section{Acknowledgements}

Experimental work was carried out in the Chemistry \& Biochemistry Department, College of Science at California State Polytechnic University in Pomona. AJ and SCES would like to acknowledge the Provost's Teacher-Scholar award for facilitating research activities.
Funding information

Funding for this research was provided by: National Science Foundation (grant No. 1800605 to Alex John; grant No. 1847926 to S. Chantal E. Stieber); US Department of Defense (grant No. W911NF-17-1- 0537 to S. Chantal E. Stieber); MENTORES PPOHA (scholarship to Jacob P. Brannon).

\section{References}

Bruker (2016). APEX2, SAINT and SADABS. Bruker AXS Inc., Madison, Wisconsin, USA.

Chakravarthy, R. D. \& Chand, D. K. (2011). J. Chem. Sci. 123, 187199.

Dolomanov, O. V., Bourhis, L. J., Gildea, R. J., Howard, J. A. K. \& Puschmann, H. (2009). J. Appl. Cryst. 42, 339-341.

Enemark, J. H., Cooney, J. J. A., Wang, J.-J. \& Holm, R. H. (2004). Chem. Rev. 104, 1175-1200.

Groom, C. R., Bruno, I. J., Lightfoot, M. P. \& Ward, S. C. (2016). Acta Cryst. B72, 171-179.

Hille, R. (1996). Chem. Rev. 96, 2757-2816.

Hille, R., Hall, J. \& Basu, P. (2014). Chem. Rev. 114, 39634038.

Hossain, M. K., Köhntopp, A., Haukka, M., Richmond, M. G., Lehtonen, A. \& Nordlander, E. (2020). Polyhedron, 178, 114312.

Mayilmurugan, R., Traar, P., Schachner, J. A., Volpe, M. \& MöschZanetti, N. C. (2013). Eur. J. Inorg. Chem. 3644-3670.

Rajan, O. A., Spence, J. T., Leman, C., Minelli, M., Sato, M., Enemark, J. H., Kroneck, P. M. H. \& Sulger, K. (1983). Inorg. Chem. 22, 30653072 . 
Roy, S., Mohanty, M., Pasayat, S., Majumder, S., Senthilguru, K., Banerjee, I., Reichelt, M., Reuter, H., Sinn, E. \& Dinda, R. (2017). J. Inorg. Biochem. 172, 110-121.

SciFinder (2021). Chemical Abstracts Service: Colombus, OH, 2010;

RN 58-08-2 (accessed September 22, 2021).

Sheldrick, G. M. (2008). Acta Cryst. A64, 112-122.

Sheldrick, G. M. (2015). Acta Cryst. A71, 3-8.
Subramanian, P., Spence, J. T., Ortega, R. \& Enemark, J. H. (1984). Inorg. Chem. 23, 2564-2572.

Whiteoak, C. J., Britovsek, G. J. P., Gibson, V. C. \& White, A. J. P. (2009). Dalton Trans. pp. 2337-2344.

Yang, H., Wang, H. \& Zhu, C. (2007). J. Org. Chem. 72, 10029-10034.

Ziegler, J. E., Du, G., Fanwick, P. E. \& Abu-Omar, M. M. (2009). Inorg. Chem. 48, 11290-11296. 


\section{supporting information}

Acta Cryst. (2022). E78, 244-250 [https://doi.org/10.1107/S2056989022000524]

Crystal structures of two dioxomolybdenum complexes stabilized by salan ligands featuring phenyl and cyclohexyl backbones

Tristhan Trieu-Tran, Stephenie N. Martinez, Jacob P. Brannon, S. Chantal E. Stieber and Alex John

Computing details

For both structures, data collection: APEX2 (Bruker, 2016); cell refinement: SAINT (Bruker, 2016); data reduction: SAINT (Bruker, 2016); program(s) used to solve structure: SHELXT (Sheldrick, 2015); program(s) used to refine structure: SHELXL (Sheldrick, 2008); molecular graphics: OLEX2 (Dolomanov et al., 2009); software used to prepare material for publication: OLEX2 (Dolomanov et al., 2009).

(6,6'-\{[(Cyclohexane-1,2-diyl)bis(azanediyl)]bis(methylene)\}bis(2,4-di-tertbutylphenolato))dioxidomolybdenum(VI) methanol disolvate (2b)

\section{Crystal data}

$\left[\mathrm{Mo}\left(\mathrm{C}_{36} \mathrm{H}_{56} \mathrm{~N}_{2} \mathrm{O}_{2}\right) \mathrm{O}_{2}\right] \cdot 2 \mathrm{CH}_{4} \mathrm{O}$

$M_{r}=740.84$

Monoclinic, $P 2{ }_{1} / n$

$a=18.4889$ (14) $\AA$

$b=10.9722(8) \AA$

$c=19.1517(14) \AA$

$\beta=94.035(2)^{\circ}$

$V=3875.6(5) \AA^{3}$

$Z=4$

Data collection

Bruker APEXII CCD diffractometer $\varphi$ and $\omega$ scans

Absorption correction: multi-scan

(SADABS; Bruker, 2016)

$T_{\min }=0.672, T_{\max }=0.750$

29075 measured reflections

Refinement

Refinement on $F^{2}$

Least-squares matrix: full

$R\left[F^{2}>2 \sigma\left(F^{2}\right)\right]=0.028$

$w R\left(F^{2}\right)=0.070$

$S=1.07$

9532 reflections

440 parameters
$F(000)=1584$

$D_{\mathrm{x}}=1.270 \mathrm{Mg} \mathrm{m}^{-3}$

Mo $K \alpha$ radiation, $\lambda=0.71073 \AA$

Cell parameters from 9945 reflections

$\theta=5.3-51.4^{\circ}$

$\mu=0.38 \mathrm{~mm}^{-1}$

$T=105 \mathrm{~K}$

Prism, clear yellow

$0.2 \times 0.18 \times 0.1 \mathrm{~mm}$

9532 independent reflections

8724 reflections with $I>2 \sigma(I)$

$R_{\text {int }}=0.026$

$\theta_{\max }=28.3^{\circ}, \theta_{\min }=5.3^{\circ}$

$h=-24 \rightarrow 24$

$k=-14 \rightarrow 14$

$l=-25 \rightarrow 25$

0 restraints

Primary atom site location: dual

Hydrogen site location: inferred from neighbouring sites

$\mathrm{H}$-atom parameters constrained

$w=1 /\left[\sigma^{2}\left(F_{0}^{2}\right)+(0.0277 P)^{2}+2.9594 P\right]$

where $P=\left(F_{\mathrm{o}}^{2}+2 F_{\mathrm{c}}^{2}\right) / 3$ 
$(\Delta / \sigma)_{\max }=0.002$

$\Delta \rho_{\min }=-0.52$ e $\AA^{-3}$

$\Delta \rho_{\max }=0.52 \mathrm{e} \AA^{-3}$

Special details

Geometry. All esds (except the esd in the dihedral angle between two 1.s. planes) are estimated using the full covariance matrix. The cell esds are taken into account individually in the estimation of esds in distances, angles and torsion angles; correlations between esds in cell parameters are only used when they are defined by crystal symmetry. An approximate (isotropic) treatment of cell esds is used for estimating esds involving l.s. planes.

Fractional atomic coordinates and isotropic or equivalent isotropic displacement parameters $\left(\AA^{2}\right)$

\begin{tabular}{|c|c|c|c|c|}
\hline & $x$ & $y$ & $z$ & $U_{\text {iso }} * / U_{\text {eq }}$ \\
\hline Mo01 & $0.59832(2)$ & $0.45373(2)$ & $0.68071(2)$ & $0.00996(4)$ \\
\hline $\mathrm{O} 1$ & $0.67906(5)$ & $0.43892(9)$ & $0.62159(5)$ & 0.01218 (19) \\
\hline $\mathrm{O} 3$ & $0.64642(6)$ & $0.52033(10)$ & $0.75027(6)$ & $0.0154(2)$ \\
\hline $\mathrm{O} 2$ & $0.51926(5)$ & $0.39987(10)$ & $0.73535(5)$ & 0.01299 (19) \\
\hline $\mathrm{O} 4$ & $0.55503(6)$ & $0.56653(10)$ & $0.63112(6)$ & $0.0166(2)$ \\
\hline N2 & $0.54408(6)$ & $0.30514(11)$ & $0.60679(6)$ & $0.0124(2)$ \\
\hline $\mathrm{H} 2$ & 0.562452 & 0.317877 & 0.559459 & $0.015^{*}$ \\
\hline N1 & $0.64967(6)$ & $0.26557(11)$ & $0.71382(6)$ & $0.0108(2)$ \\
\hline H1 & 0.621681 & 0.233132 & 0.752592 & $0.013^{*}$ \\
\hline O5 & $0.44789(6)$ & $0.68127(12)$ & $0.54536(6)$ & 0.0239 \\
\hline H5A & 0.481126 & 0.646691 & 0.570127 & $0.036^{*}$ \\
\hline $\mathrm{C} 22$ & $0.46379(8)$ & $0.32549(14)$ & $0.59954(7)$ & $0.0142(3)$ \\
\hline $\mathrm{H} 22 \mathrm{~A}$ & 0.442308 & 0.276831 & 0.559782 & $0.017 *$ \\
\hline $\mathrm{H} 22 \mathrm{~B}$ & 0.453789 & 0.412566 & 0.589333 & $0.017 *$ \\
\hline $\mathrm{C} 3$ & $0.84869(7)$ & $0.31009(13)$ & $0.70189(7)$ & 0.0111 \\
\hline $\mathrm{H} 3$ & 0.864314 & 0.255829 & 0.738559 & $0.013 *$ \\
\hline $\mathrm{C} 5$ & $0.87410(7)$ & $0.43712(13)$ & $0.60581(7)$ & 0.0121 \\
\hline H5 & 0.908405 & 0.472482 & 0.577181 & $0.015^{*}$ \\
\hline $\mathrm{C} 24$ & $0.46022(7)$ & $0.32631(13)$ & $0.73127(7)$ & 0.0119 \\
\hline $\mathrm{C} 1$ & $0.75049(7)$ & $0.41320(13)$ & $0.63556(7)$ & $0.0102(2)$ \\
\hline $\mathrm{C} 25$ & $0.43157(7)$ & $0.28483(13)$ & $0.79316(7)$ & 0.0126 \\
\hline $\mathrm{C} 2$ & $0.77461(7)$ & $0.33656(13)$ & $0.69077(7)$ & $0.0108(2)$ \\
\hline C33 & $0.46569(8)$ & $0.32001(14)$ & $0.86602(7)$ & $0.0142(3)$ \\
\hline $\mathrm{C} 4$ & $0.89997(7)$ & $0.36090(13)$ & $0.66085(7)$ & 0.0118 \\
\hline $\mathrm{C} 26$ & $0.36957(8)$ & $0.21086(14)$ & $0.78600(8)$ & $0.0146(3)$ \\
\hline $\mathrm{H} 26$ & 0.348850 & 0.184092 & 0.827336 & $0.017 *$ \\
\hline $\mathrm{C} 23$ & $0.42907(8)$ & $0.28965(14)$ & $0.66554(7)$ & $0.0142(3)$ \\
\hline C9 & $0.74008(9)$ & $0.66304(14)$ & $0.55600(9)$ & $0.0201(3)$ \\
\hline H9A & 0.697246 & 0.641207 & 0.580670 & $0.030 *$ \\
\hline H9B & 0.725631 & 0.716410 & 0.516425 & $0.030 *$ \\
\hline $\mathrm{H} 9 \mathrm{C}$ & 0.775058 & 0.705636 & 0.588184 & $0.030^{*}$ \\
\hline C6 & $0.80076(7)$ & $0.46405(12)$ & $0.59066(7)$ & $0.0111(2)$ \\
\hline $\mathrm{C} 15$ & $0.72567(7)$ & $0.28252(13)$ & $0.74267(7)$ & $0.0110(2)$ \\
\hline H15A & 0.745494 & 0.202582 & 0.758523 & $0.013 *$ \\
\hline H15B & 0.725879 & 0.336533 & 0.784094 & $0.013 *$ \\
\hline $\mathrm{C} 7$ & $0.77512(8)$ & $0.54657(13)$ & $0.52888(7)$ & $0.0128(3)$ \\
\hline $\mathrm{C} 10$ & $0.83846(8)$ & $0.58516(15)$ & $0.48592(8)$ & 0.0170 \\
\hline
\end{tabular}




\begin{tabular}{|c|c|c|c|c|}
\hline H10A & 0.820131 & 0.636333 & 0.446633 & $0.025^{*}$ \\
\hline H10B & 0.861715 & 0.512455 & 0.467948 & $0.025^{*}$ \\
\hline $\mathrm{H} 10 \mathrm{C}$ & 0.873925 & 0.631277 & 0.515788 & $0.025^{*}$ \\
\hline C16 & $0.64240(8)$ & $0.17496(13)$ & $0.65555(7)$ & $0.0141(3)$ \\
\hline H16 & 0.672705 & 0.202504 & 0.617289 & $0.017^{*}$ \\
\hline $\mathrm{C} 21$ & $0.66673(8)$ & $0.04744(13)$ & $0.67866(8)$ & $0.0160(3)$ \\
\hline $\mathrm{H} 21 \mathrm{~A}$ & 0.642144 & 0.024197 & 0.720976 & $0.019^{*}$ \\
\hline $\mathrm{H} 21 \mathrm{~B}$ & 0.719628 & 0.047899 & 0.691019 & $0.019^{*}$ \\
\hline $\mathrm{C} 27$ & $0.33673(8)$ & $0.17452(14)$ & $0.72175(8)$ & $0.0157(3)$ \\
\hline $\mathrm{C} 28$ & $0.36837(8)$ & $0.21471(14)$ & $0.66180(8)$ & $0.0162(3)$ \\
\hline $\mathrm{H} 28$ & 0.347797 & 0.190093 & 0.617174 & $0.019 *$ \\
\hline C11 & $0.98156(7)$ & $0.33692(13)$ & $0.67445(7)$ & $0.0129(3)$ \\
\hline $\mathrm{C} 36$ & $0.54757(8)$ & $0.29016(15)$ & $0.87359(8)$ & $0.0194(3)$ \\
\hline H36A & 0.555113 & 0.205208 & 0.860075 & $0.029 *$ \\
\hline H36B & 0.565991 & 0.302178 & 0.922347 & $0.029 *$ \\
\hline $\mathrm{H} 36 \mathrm{C}$ & 0.573525 & 0.344170 & 0.843165 & $0.029 *$ \\
\hline C19 & $0.56900(10)$ & $-0.04481(15)$ & $0.59694(9)$ & $0.0219(3)$ \\
\hline H19A & 0.539944 & -0.068313 & 0.636294 & $0.026^{*}$ \\
\hline H19B & 0.559450 & -0.104765 & 0.558777 & $0.026^{*}$ \\
\hline $\mathrm{C} 20$ & $0.64927(9)$ & $-0.04664(15)$ & $0.62109(9)$ & $0.0224(3)$ \\
\hline $\mathrm{H} 20 \mathrm{~A}$ & 0.678364 & -0.029062 & 0.580807 & $0.027 *$ \\
\hline H20B & 0.662703 & -0.128871 & 0.638897 & $0.027^{*}$ \\
\hline C14 & $1.02015(9)$ & $0.45629(15)$ & $0.69599(9)$ & $0.0222(3)$ \\
\hline $\mathrm{H} 14 \mathrm{~A}$ & 1.012181 & 0.516351 & 0.658365 & $0.033 *$ \\
\hline H14B & 1.072247 & 0.441111 & 0.704617 & $0.033^{*}$ \\
\hline $\mathrm{H} 14 \mathrm{C}$ & 1.000677 & 0.487651 & 0.738759 & $0.033 *$ \\
\hline C34 & $0.43001(10)$ & $0.25202(16)$ & $0.92482(8)$ & $0.0229(3)$ \\
\hline H34A & 0.378309 & 0.272408 & 0.923167 & $0.034^{*}$ \\
\hline H34B & 0.453306 & 0.276340 & 0.970230 & $0.034^{*}$ \\
\hline $\mathrm{H} 34 \mathrm{C}$ & 0.435662 & 0.163973 & 0.918539 & $0.034 *$ \\
\hline C12 & $0.99721(8)$ & $0.24308(14)$ & $0.73274(8)$ & $0.0161(3)$ \\
\hline $\mathrm{H} 12 \mathrm{~A}$ & 0.979753 & 0.274088 & 0.776503 & $0.024 *$ \\
\hline H12B & 1.049566 & 0.228556 & 0.739032 & $0.024 *$ \\
\hline $\mathrm{H} 12 \mathrm{C}$ & 0.972342 & 0.166547 & 0.720004 & $0.024 *$ \\
\hline $\mathrm{C} 8$ & $0.72060(9)$ & $0.47859(16)$ & $0.47828(8)$ & $0.0193(3)$ \\
\hline $\mathrm{H} 8 \mathrm{~A}$ & 0.677274 & 0.458088 & 0.502541 & $0.029 *$ \\
\hline H8B & 0.742917 & 0.403623 & 0.462137 & $0.029 *$ \\
\hline $\mathrm{H} 8 \mathrm{C}$ & 0.706920 & 0.530810 & 0.437985 & $0.029 *$ \\
\hline $\mathrm{C} 35$ & $0.45589(9)$ & $0.45675(14)$ & $0.87842(8)$ & $0.0205(3)$ \\
\hline $\mathrm{H} 35 \mathrm{~A}$ & 0.476047 & 0.502693 & 0.840440 & $0.031 *$ \\
\hline H35B & 0.481207 & 0.479742 & 0.923160 & $0.031 *$ \\
\hline $\mathrm{H} 35 \mathrm{C}$ & 0.404140 & 0.475293 & 0.879611 & $0.031 *$ \\
\hline C17 & $0.56291(8)$ & $0.17702(14)$ & $0.62828(8)$ & $0.0144(3)$ \\
\hline H17 & 0.533304 & 0.155719 & 0.668282 & $0.017^{*}$ \\
\hline C13 & $1.01268(8)$ & $0.28886(15)$ & $0.60759(8)$ & $0.0186(3)$ \\
\hline $\mathrm{H} 13 \mathrm{~A}$ & 0.987537 & 0.213576 & 0.592770 & $0.028 *$ \\
\hline H13B & 1.064551 & 0.272195 & 0.616823 & $0.028 *$ \\
\hline $\mathrm{H} 13 \mathrm{C}$ & 1.005854 & 0.350083 & 0.570454 & $0.028 *$ \\
\hline
\end{tabular}




$\begin{array}{lllll}\text { C18 } & 0.54628(9) & 0.08268(15) & 0.57084(8) & 0.0193(3) \\ \text { H18A } & 0.572711 & 0.103741 & 0.529292 & 0.023^{*} \\ \text { H18B } & 0.493712 & 0.083263 & 0.556814 & 0.023^{*} \\ \text { C29 } & 0.26833(8) & 0.09489(15) & 0.71510(9) & 0.0185(3) \\ \text { C30 } & 0.28065(10) & -0.01727(16) & 0.66956(10) & 0.0261(4) \\ \text { H30A } & 0.290396 & 0.009075 & 0.622240 & 0.039^{*} \\ \text { H30B } & 0.237252 & -0.068785 & 0.667349 & 0.039^{*} \\ \text { H30C } & 0.322176 & -0.063725 & 0.690021 & 0.039^{*} \\ \text { C37 } & 0.40015(9) & 0.74080(18) & 0.58931(9) & 0.0263(4) \\ \text { H37A } & 0.356503 & 0.767075 & 0.561340 & 0.039^{*} \\ \text { H37B } & 0.424456 & 0.812034 & 0.611086 & 0.039^{*} \\ \text { H37C } & 0.386558 & 0.684354 & 0.625822 & 0.039^{*} \\ \text { C32 } & 0.24605(10) & 0.04964(19) & 0.78619(10) & 0.0299(4) \\ \text { H32A } & 0.285986 & 0.003025 & 0.809534 & 0.045^{*} \\ \text { H32B } & 0.203172 & -0.002637 & 0.779172 & 0.045^{*} \\ \text { H32C } & 0.234725 & 0.119593 & 0.815306 & 0.045^{*} \\ \text { C31 } & 0.20611(9) & 0.17193(17) & 0.68148(12) & 0.0314(4) \\ \text { H31A } & 0.200184 & 0.245472 & 0.709543 & 0.047^{*} \\ \text { H31B } & 0.161139 & 0.124390 & 0.679387 & 0.047^{*} \\ \text { H31C } & 0.217224 & 0.195183 & 0.633999 & 0.047^{*} \\ \text { O0AA } & 0.78475(9) & 0.15803(16) & 0.53746(9) & 0.0475(4) \\ \text { H0AA } & 0.753457 & 0.134959 & 0.506334 & 0.071^{*} \\ \text { C38 } & 0.83042(13) & 0.0590(2) & 0.55795(13) & 0.0447(5) \\ \text { H38A } & 0.809561 & -0.016705 & 0.538274 & 0.067^{*} \\ \text { H38B } & 0.834867 & 0.053270 & 0.609140 & 0.067^{*} \\ \text { H38C } & 0.878466 & 0.071852 & 0.540644 & 0.067^{*}\end{array}$

Atomic displacement parameters $\left(\AA^{2}\right)$

\begin{tabular}{lllllll}
\hline & $U^{11}$ & $U^{22}$ & $U^{33}$ & $U^{12}$ & $U^{13}$ & $U^{23}$ \\
\hline Mo01 & $0.00740(6)$ & $0.01008(6)$ & $0.01251(6)$ & $0.00059(4)$ & $0.00143(4)$ & $0.00055(4)$ \\
O1 & $0.0078(4)$ & $0.0146(5)$ & $0.0143(5)$ & $0.0017(4)$ & $0.0018(4)$ & $0.0034(4)$ \\
O3 & $0.0134(5)$ & $0.0137(5)$ & $0.0190(5)$ & $-0.0010(4)$ & $0.0010(4)$ & $-0.0023(4)$ \\
O2 & $0.0102(4)$ & $0.0154(5)$ & $0.0135(5)$ & $-0.0020(4)$ & $0.0023(4)$ & $-0.0009(4)$ \\
O4 & $0.0135(5)$ & $0.0168(5)$ & $0.0197(5)$ & $0.0044(4)$ & $0.0030(4)$ & $0.0037(4)$ \\
N2 & $0.0099(5)$ & $0.0152(6)$ & $0.0119(5)$ & $0.0022(5)$ & $0.0000(4)$ & $0.0012(4)$ \\
N1 & $0.0079(5)$ & $0.0117(5)$ & $0.0127(5)$ & $-0.0015(4)$ & $0.0006(4)$ & $0.0002(4)$ \\
O5 & $0.0216(6)$ & $0.0340(7)$ & $0.0158(5)$ & $0.0087(5)$ & $-0.0007(4)$ & $0.0025(5)$ \\
C22 & $0.0102(6)$ & $0.0187(7)$ & $0.0133(6)$ & $-0.0004(5)$ & $-0.0014(5)$ & $0.0016(5)$ \\
C3 & $0.0104(6)$ & $0.0096(6)$ & $0.0131(6)$ & $0.0002(5)$ & $-0.0002(5)$ & $0.0003(5)$ \\
C5 & $0.0101(6)$ & $0.0122(6)$ & $0.0144(6)$ & $-0.0018(5)$ & $0.0029(5)$ & $0.0005(5)$ \\
C24 & $0.0072(6)$ & $0.0128(6)$ & $0.0156(6)$ & $0.0009(5)$ & $0.0004(5)$ & $0.0007(5)$ \\
C1 & $0.0077(6)$ & $0.0100(6)$ & $0.0131(6)$ & $-0.0003(5)$ & $0.0013(5)$ & $-0.0009(5)$ \\
C25 & $0.0104(6)$ & $0.0125(6)$ & $0.0148(6)$ & $0.0023(5)$ & $0.0006(5)$ & $0.0009(5)$ \\
C2 & $0.0094(6)$ & $0.0099(6)$ & $0.0131(6)$ & $-0.0020(5)$ & $0.0014(5)$ & $-0.0002(5)$ \\
C33 & $0.0157(7)$ & $0.0140(7)$ & $0.0130(6)$ & $0.0010(5)$ & $0.0022(5)$ & $0.0004(5)$ \\
C4 & $0.0090(6)$ & $0.0114(6)$ & $0.0150(6)$ & $-0.0002(5)$ & $0.0010(5)$ & $-0.0013(5)$ \\
C26 & $0.0113(6)$ & $0.0144(7)$ & $0.0185(7)$ & $0.0013(5)$ & $0.0042(5)$ & $0.0029(5)$
\end{tabular}




$\begin{array}{lllllll}\text { C23 } & 0.0115(6) & 0.0178(7) & 0.0133(6) & 0.0002(5) & -0.0001(5) & 0.0022(5) \\ \text { C9 } & 0.0236(8) & 0.0144(7) & 0.0231(7) & 0.0052(6) & 0.0068(6) & 0.0051(6) \\ \text { C6 } & 0.0108(6) & 0.0097(6) & 0.0129(6) & -0.0004(5) & 0.0018(5) & 0.0004(5) \\ \text { C15 } & 0.0083(6) & 0.0126(6) & 0.0120(6) & -0.0017(5) & -0.0004(5) & 0.0018(5) \\ \text { C7 } & 0.0116(6) & 0.0133(6) & 0.0135(6) & 0.0002(5) & 0.0019(5) & 0.0028(5) \\ \text { C10 } & 0.0149(7) & 0.0197(7) & 0.0166(7) & -0.0022(6) & 0.0035(5) & 0.0055(6) \\ \text { C16 } & 0.0140(6) & 0.0130(7) & 0.0150(6) & 0.0000(5) & -0.0013(5) & -0.0017(5) \\ \text { C21 } & 0.0157(7) & 0.0118(7) & 0.0197(7) & 0.0019(5) & -0.0041(5) & -0.0005(5) \\ \text { C27 } & 0.0093(6) & 0.0157(7) & 0.0219(7) & -0.0005(5) & 0.0003(5) & 0.0025(6) \\ \text { C28 } & 0.0123(6) & 0.0189(7) & 0.0169(7) & -0.0008(6) & -0.0028(5) & 0.0014(6) \\ \text { C11 } & 0.0077(6) & 0.0140(7) & 0.0169(6) & 0.0000(5) & 0.0006(5) & 0.0010(5) \\ \text { C36 } & 0.0177(7) & 0.0221(8) & 0.0177(7) & 0.0026(6) & -0.0039(6) & 0.0001(6) \\ \text { C19 } & 0.0274(8) & 0.0155(7) & 0.0218(7) & -0.0022(6) & -0.0057(6) & -0.0017(6) \\ \text { C20 } & 0.0258(8) & 0.0136(7) & 0.0269(8) & 0.0028(6) & -0.0046(6) & -0.0039(6) \\ \text { C14 } & 0.0144(7) & 0.0178(7) & 0.0336(9) & -0.0045(6) & -0.0042(6) & -0.0005(6) \\ \text { C34 } & 0.0301(9) & 0.0251(8) & 0.0138(7) & -0.0048(7) & 0.0042(6) & 0.0027(6) \\ \text { C12 } & 0.0118(6) & 0.0191(7) & 0.0175(7) & 0.0027(6) & 0.0011(5) & 0.0035(6) \\ \text { C8 } & 0.0168(7) & 0.0261(8) & 0.0145(7) & -0.0049(6) & -0.0012(5) & 0.0026(6) \\ \text { C35 } & 0.0274(8) & 0.0161(7) & 0.0183(7) & 0.0028(6) & 0.0031(6) & -0.0010(6) \\ \text { C17 } & 0.0141(6) & 0.0133(7) & 0.0156(7) & -0.0007(5) & -0.0006(5) & 0.0005(5) \\ \text { C13 } & 0.0134(7) & 0.0242(8) & 0.0185(7) & 0.0046(6) & 0.0033(5) & 0.0037(6) \\ \text { C18 } & 0.0236(8) & 0.0161(7) & 0.0171(7) & 0.0005(6) & -0.0068(6) & -0.0025(6) \\ \text { C29 } & 0.0103(6) & 0.0180(7) & 0.0270(8) & -0.0030(6) & 0.0014(6) & 0.0012(6) \\ \text { C30 } & 0.0221(8) & 0.0218(8) & 0.0345(9) & -0.0053(7) & 0.0031(7) & -0.0032(7) \\ \text { C37 } & 0.0202(8) & 0.0358(10) & 0.0230(8) & -0.0026(7) & 0.0027(6) & -0.0067(7) \\ \text { C32 } & 0.0226(8) & 0.0354(10) & 0.0324(9) & -0.0141(8) & 0.0084(7) & 0.0008(8) \\ \text { C31 } & 0.0135(7) & 0.0230(9) & 0.0563(12) & -0.0015(7) & -0.0078(8) & 0.0031(8) \\ \text { O0AA } & 0.0424(9) & 0.0457(9) & 0.0550(10) & 0.0012(8) & 0.0073(7) & -0.0082(8) \\ \text { C38 } & 0.0409(12) & 0.0465(13) & 0.0479(13) & 0.0002(10) & 0.0118(10) & 0.0003(10)\end{array}$

Geometric parameters $\left(\AA,{ }^{\circ}\right)$

\begin{tabular}{|c|c|c|c|}
\hline Mo01-O1 & $1.9428(10)$ & $\mathrm{C} 27-\mathrm{C} 28$ & $1.396(2)$ \\
\hline Mo01-O3 & $1.7125(10)$ & $\mathrm{C} 27-\mathrm{C} 29$ & $1.535(2)$ \\
\hline Mo01-O2 & $1.9484(10)$ & $\mathrm{C} 28-\mathrm{H} 28$ & 0.9500 \\
\hline Mo01-O4 & $1.7226(11)$ & $\mathrm{C} 11-\mathrm{C} 14$ & $1.534(2)$ \\
\hline Mo01-N2 & $2.3384(12)$ & $\mathrm{C} 11-\mathrm{C} 12$ & $1.531(2)$ \\
\hline Mo01-N1 & $2.3412(12)$ & $\mathrm{C} 11-\mathrm{C} 13$ & $1.534(2)$ \\
\hline $\mathrm{O} 1-\mathrm{C} 1$ & $1.3586(16)$ & C36-H36A & 0.9800 \\
\hline $\mathrm{O} 2-\mathrm{C} 24$ & $1.3554(17)$ & С $36-\mathrm{H} 36 \mathrm{~B}$ & 0.9800 \\
\hline $\mathrm{N} 2-\mathrm{H} 2$ & 1.0000 & C36-H36C & 0.9800 \\
\hline $\mathrm{N} 2-\mathrm{C} 22$ & $1.4979(18)$ & C19-H19A & 0.9900 \\
\hline $\mathrm{N} 2-\mathrm{C} 17$ & 1.4989 (19) & С19-H19B & 0.9900 \\
\hline $\mathrm{N} 1-\mathrm{H} 1$ & 1.0000 & $\mathrm{C} 19-\mathrm{C} 20$ & $1.523(2)$ \\
\hline $\mathrm{N} 1-\mathrm{C} 15$ & $1.4850(17)$ & $\mathrm{C} 19-\mathrm{C} 18$ & $1.534(2)$ \\
\hline $\mathrm{N} 1-\mathrm{C} 16$ & $1.4935(18)$ & $\mathrm{C} 20-\mathrm{H} 20 \mathrm{~A}$ & 0.9900 \\
\hline $\mathrm{O} 5-\mathrm{H} 5 \mathrm{~A}$ & 0.8400 & $\mathrm{C} 20-\mathrm{H} 20 \mathrm{~B}$ & 0.9900 \\
\hline $\mathrm{O} 5-\mathrm{C} 37$ & $1.421(2)$ & $\mathrm{C} 14-\mathrm{H} 14 \mathrm{~A}$ & 0.9800 \\
\hline
\end{tabular}




\begin{tabular}{|c|c|c|c|}
\hline $\mathrm{C} 22-\mathrm{H} 22 \mathrm{~A}$ & 0.9900 & $\mathrm{C} 14-\mathrm{H} 14 \mathrm{~B}$ & 0.9800 \\
\hline $\mathrm{C} 22-\mathrm{H} 22 \mathrm{~B}$ & 0.9900 & $\mathrm{C} 14-\mathrm{H} 14 \mathrm{C}$ & 0.9800 \\
\hline $\mathrm{C} 22-\mathrm{C} 23$ & $1.510(2)$ & $\mathrm{C} 34-\mathrm{H} 34 \mathrm{~A}$ & 0.9800 \\
\hline $\mathrm{C} 3-\mathrm{H} 3$ & 0.9500 & C34-H34B & 0.9800 \\
\hline $\mathrm{C} 3-\mathrm{C} 2$ & $1.4016(18)$ & $\mathrm{C} 34-\mathrm{H} 34 \mathrm{C}$ & 0.9800 \\
\hline $\mathrm{C} 3-\mathrm{C} 4$ & $1.3902(19)$ & $\mathrm{C} 12-\mathrm{H} 12 \mathrm{~A}$ & 0.9800 \\
\hline $\mathrm{C} 5-\mathrm{H} 5$ & 0.9500 & $\mathrm{C} 12-\mathrm{H} 12 \mathrm{~B}$ & 0.9800 \\
\hline $\mathrm{C} 5-\mathrm{C} 4$ & $1.4029(19)$ & $\mathrm{C} 12-\mathrm{H} 12 \mathrm{C}$ & 0.9800 \\
\hline $\mathrm{C} 5-\mathrm{C} 6$ & $1.3982(19)$ & C8-H8A & 0.9800 \\
\hline $\mathrm{C} 24-\mathrm{C} 25$ & $1.407(2)$ & $\mathrm{C} 8-\mathrm{H} 8 \mathrm{~B}$ & 0.9800 \\
\hline $\mathrm{C} 24-\mathrm{C} 23$ & 1.4057 (19) & $\mathrm{C} 8-\mathrm{H} 8 \mathrm{C}$ & 0.9800 \\
\hline $\mathrm{C} 1-\mathrm{C} 2$ & $1.3992(19)$ & $\mathrm{C} 35-\mathrm{H} 35 \mathrm{~A}$ & 0.9800 \\
\hline $\mathrm{C} 1-\mathrm{C} 6$ & $1.4238(19)$ & $\mathrm{C} 35-\mathrm{H} 35 \mathrm{~B}$ & 0.9800 \\
\hline $\mathrm{C} 25-\mathrm{C} 33$ & $1.5396(19)$ & $\mathrm{C} 35-\mathrm{H} 35 \mathrm{C}$ & 0.9800 \\
\hline $\mathrm{C} 25-\mathrm{C} 26$ & $1.403(2)$ & C17-H17 & 1.0000 \\
\hline $\mathrm{C} 2-\mathrm{C} 15$ & $1.5117(18)$ & $\mathrm{C} 17-\mathrm{C} 18$ & $1.526(2)$ \\
\hline $\mathrm{C} 33-\mathrm{C} 36$ & $1.546(2)$ & $\mathrm{C} 13-\mathrm{H} 13 \mathrm{~A}$ & 0.9800 \\
\hline $\mathrm{C} 33-\mathrm{C} 34$ & $1.538(2)$ & $\mathrm{C} 13-\mathrm{H} 13 \mathrm{~B}$ & 0.9800 \\
\hline $\mathrm{C} 33-\mathrm{C} 35$ & $1.532(2)$ & $\mathrm{C} 13-\mathrm{H} 13 \mathrm{C}$ & 0.9800 \\
\hline $\mathrm{C} 4-\mathrm{C} 11$ & $1.5353(19)$ & $\mathrm{C} 18-\mathrm{H} 18 \mathrm{~A}$ & 0.9900 \\
\hline $\mathrm{C} 26-\mathrm{H} 26$ & 0.9500 & C18-H18B & 0.9900 \\
\hline $\mathrm{C} 26-\mathrm{C} 27$ & $1.391(2)$ & $\mathrm{C} 29-\mathrm{C} 30$ & $1.535(2)$ \\
\hline $\mathrm{C} 23-\mathrm{C} 28$ & $1.389(2)$ & $\mathrm{C} 29-\mathrm{C} 32$ & $1.533(2)$ \\
\hline C9-H9A & 0.9800 & $\mathrm{C} 29-\mathrm{C} 31$ & $1.532(2)$ \\
\hline C9-H9B & 0.9800 & $\mathrm{C} 30-\mathrm{H} 30 \mathrm{~A}$ & 0.9800 \\
\hline C9-H9C & 0.9800 & $\mathrm{C} 30-\mathrm{H} 30 \mathrm{~B}$ & 0.9800 \\
\hline $\mathrm{C} 9-\mathrm{C} 7$ & $1.539(2)$ & $\mathrm{C} 30-\mathrm{H} 30 \mathrm{C}$ & 0.9800 \\
\hline $\mathrm{C} 6-\mathrm{C} 7$ & $1.5380(19)$ & $\mathrm{C} 37-\mathrm{H} 37 \mathrm{~A}$ & 0.9800 \\
\hline C15-H15A & 0.9900 & C37-H37B & 0.9800 \\
\hline C15-H15B & 0.9900 & C37-H37C & 0.9800 \\
\hline $\mathrm{C} 7-\mathrm{C} 10$ & $1.537(2)$ & $\mathrm{C} 32-\mathrm{H} 32 \mathrm{~A}$ & 0.9800 \\
\hline $\mathrm{C} 7-\mathrm{C} 8$ & $1.541(2)$ & $\mathrm{C} 32-\mathrm{H} 32 \mathrm{~B}$ & 0.9800 \\
\hline $\mathrm{C} 10-\mathrm{H} 10 \mathrm{~A}$ & 0.9800 & $\mathrm{C} 32-\mathrm{H} 32 \mathrm{C}$ & 0.9800 \\
\hline $\mathrm{C} 10-\mathrm{H} 10 \mathrm{~B}$ & 0.9800 & $\mathrm{C} 31-\mathrm{H} 31 \mathrm{~A}$ & 0.9800 \\
\hline $\mathrm{C} 10-\mathrm{H} 10 \mathrm{C}$ & 0.9800 & $\mathrm{C} 31-\mathrm{H} 31 \mathrm{~B}$ & 0.9800 \\
\hline $\mathrm{C} 16-\mathrm{H} 16$ & 1.0000 & $\mathrm{C} 31-\mathrm{H} 31 \mathrm{C}$ & 0.9800 \\
\hline $\mathrm{C} 16-\mathrm{C} 21$ & $1.526(2)$ & O0AA-H0AA & 0.8400 \\
\hline $\mathrm{C} 16-\mathrm{C} 17$ & $1.5250(19)$ & $\mathrm{O} 0 \mathrm{AA}-\mathrm{C} 38$ & $1.415(3)$ \\
\hline $\mathrm{C} 21-\mathrm{H} 21 \mathrm{~A}$ & 0.9900 & $\mathrm{C} 38-\mathrm{H} 38 \mathrm{~A}$ & 0.9800 \\
\hline $\mathrm{C} 21-\mathrm{H} 21 \mathrm{~B}$ & 0.9900 & $\mathrm{C} 38-\mathrm{H} 38 \mathrm{~B}$ & 0.9800 \\
\hline $\mathrm{C} 21-\mathrm{C} 20$ & $1.528(2)$ & $\mathrm{C} 38-\mathrm{H} 38 \mathrm{C}$ & 0.9800 \\
\hline $\mathrm{O} 1-\mathrm{Mo} 01-\mathrm{O} 2$ & $157.49(4)$ & $\mathrm{C} 23-\mathrm{C} 28-\mathrm{H} 28$ & 119.1 \\
\hline $\mathrm{O} 1-\mathrm{Mo} 01-\mathrm{N} 2$ & $84.45(4)$ & $\mathrm{C} 27-\mathrm{C} 28-\mathrm{H} 28$ & 119.1 \\
\hline $\mathrm{O} 1-\mathrm{Mo} 01-\mathrm{N} 1$ & $76.73(4)$ & $\mathrm{C} 14-\mathrm{C} 11-\mathrm{C} 4$ & $109.40(12)$ \\
\hline $\mathrm{O} 3-\mathrm{Mo} 01-\mathrm{O} 1$ & $96.36(5)$ & $\mathrm{C} 12-\mathrm{C} 11-\mathrm{C} 4$ & $111.84(12)$ \\
\hline $\mathrm{O} 3-\mathrm{Mo} 01-\mathrm{O} 2$ & $94.58(5)$ & $\mathrm{C} 12-\mathrm{C} 11-\mathrm{C} 14$ & $108.51(12)$ \\
\hline $\mathrm{O} 3-\mathrm{Mo01-O} 4$ & $108.55(5)$ & $\mathrm{C} 12-\mathrm{C} 11-\mathrm{C} 13$ & $108.34(12)$ \\
\hline
\end{tabular}




\begin{tabular}{|c|c|c|c|}
\hline $\mathrm{O} 3-\mathrm{Mo} 01-\mathrm{N} 2$ & $161.00(5)$ & $\mathrm{C} 13-\mathrm{C} 11-\mathrm{C} 4$ & $109.83(11)$ \\
\hline $\mathrm{O} 3-\mathrm{Mo} 01-\mathrm{N} 1$ & $89.26(5)$ & $\mathrm{C} 13-\mathrm{C} 11-\mathrm{C} 14$ & $108.87(13)$ \\
\hline $\mathrm{O} 2-\mathrm{Mo} 01-\mathrm{N} 2$ & $78.91(4)$ & $\mathrm{C} 33-\mathrm{C} 36-\mathrm{H} 36 \mathrm{~A}$ & 109.5 \\
\hline $\mathrm{O} 2-\mathrm{Mo} 01-\mathrm{N} 1$ & $83.82(4)$ & $\mathrm{C} 33-\mathrm{C} 36-\mathrm{H} 36 \mathrm{~B}$ & 109.5 \\
\hline $\mathrm{O} 4-\mathrm{Mo} 01-\mathrm{O} 1$ & $94.88(5)$ & $\mathrm{C} 33-\mathrm{C} 36-\mathrm{H} 36 \mathrm{C}$ & 109.5 \\
\hline $\mathrm{O} 4-\mathrm{Mo} 01-\mathrm{O} 2$ & $100.19(5)$ & $\mathrm{H} 36 \mathrm{~A}-\mathrm{C} 36-\mathrm{H} 36 \mathrm{~B}$ & 109.5 \\
\hline $\mathrm{O} 4-\mathrm{Mo} 01-\mathrm{N} 2$ & $90.24(5)$ & $\mathrm{H} 36 \mathrm{~A}-\mathrm{C} 36-\mathrm{H} 36 \mathrm{C}$ & 109.5 \\
\hline $\mathrm{O} 4-\mathrm{Mo} 01-\mathrm{N} 1$ & $161.21(5)$ & $\mathrm{H} 36 \mathrm{~B}-\mathrm{C} 36-\mathrm{H} 36 \mathrm{C}$ & 109.5 \\
\hline $\mathrm{N} 2-\mathrm{Mo} 01-\mathrm{N} 1$ & $72.40(4)$ & $\mathrm{H} 19 \mathrm{~A}-\mathrm{C} 19-\mathrm{H} 19 \mathrm{~B}$ & 108.1 \\
\hline $\mathrm{C} 1-\mathrm{O} 1-\mathrm{Mo0} 1$ & $132.75(9)$ & $\mathrm{C} 20-\mathrm{C} 19-\mathrm{H} 19 \mathrm{~A}$ & 109.6 \\
\hline $\mathrm{C} 24-\mathrm{O} 2-\mathrm{Mo0} 1$ & $141.38(9)$ & $\mathrm{C} 20-\mathrm{C} 19-\mathrm{H} 19 \mathrm{~B}$ & 109.6 \\
\hline $\mathrm{Mo} 01-\mathrm{N} 2-\mathrm{H} 2$ & 107.0 & $\mathrm{C} 20-\mathrm{C} 19-\mathrm{C} 18$ & $110.46(13)$ \\
\hline $\mathrm{C} 22-\mathrm{N} 2-\mathrm{Mo0} 1$ & $109.40(9)$ & $\mathrm{C} 18-\mathrm{C} 19-\mathrm{H} 19 \mathrm{~A}$ & 109.6 \\
\hline $\mathrm{C} 22-\mathrm{N} 2-\mathrm{H} 2$ & 107.0 & $\mathrm{C} 18-\mathrm{C} 19-\mathrm{H} 19 \mathrm{~B}$ & 109.6 \\
\hline $\mathrm{C} 22-\mathrm{N} 2-\mathrm{C} 17$ & $112.01(11)$ & $\mathrm{C} 21-\mathrm{C} 20-\mathrm{H} 20 \mathrm{~A}$ & 109.4 \\
\hline $\mathrm{C} 17-\mathrm{N} 2-\mathrm{Mo0} 1$ & $113.97(8)$ & $\mathrm{C} 21-\mathrm{C} 20-\mathrm{H} 20 \mathrm{~B}$ & 109.4 \\
\hline $\mathrm{C} 17-\mathrm{N} 2-\mathrm{H} 2$ & 107.0 & $\mathrm{C} 19-\mathrm{C} 20-\mathrm{C} 21$ & $111.22(14)$ \\
\hline Mo01-N1-H1 & 107.1 & $\mathrm{C} 19-\mathrm{C} 20-\mathrm{H} 20 \mathrm{~A}$ & 109.4 \\
\hline $\mathrm{C} 15-\mathrm{N} 1-\mathrm{Mo0} 1$ & $110.21(8)$ & $\mathrm{C} 19-\mathrm{C} 20-\mathrm{H} 20 \mathrm{~B}$ & 109.4 \\
\hline $\mathrm{C} 15-\mathrm{N} 1-\mathrm{H} 1$ & 107.1 & $\mathrm{H} 20 \mathrm{~A}-\mathrm{C} 20-\mathrm{H} 20 \mathrm{~B}$ & 108.0 \\
\hline $\mathrm{C} 15-\mathrm{N} 1-\mathrm{C} 16$ & $113.23(11)$ & $\mathrm{C} 11-\mathrm{C} 14-\mathrm{H} 14 \mathrm{~A}$ & 109.5 \\
\hline $\mathrm{C} 16-\mathrm{N} 1-\mathrm{Mo0} 1$ & $111.79(8)$ & $\mathrm{C} 11-\mathrm{C} 14-\mathrm{H} 14 \mathrm{~B}$ & 109.5 \\
\hline $\mathrm{C} 16-\mathrm{N} 1-\mathrm{H} 1$ & 107.1 & $\mathrm{C} 11-\mathrm{C} 14-\mathrm{H} 14 \mathrm{C}$ & 109.5 \\
\hline $\mathrm{C} 37-\mathrm{O} 5-\mathrm{H} 5 \mathrm{~A}$ & 109.5 & $\mathrm{H} 14 \mathrm{~A}-\mathrm{C} 14-\mathrm{H} 14 \mathrm{~B}$ & 109.5 \\
\hline $\mathrm{N} 2-\mathrm{C} 22-\mathrm{H} 22 \mathrm{~A}$ & 109.4 & $\mathrm{H} 14 \mathrm{~A}-\mathrm{C} 14-\mathrm{H} 14 \mathrm{C}$ & 109.5 \\
\hline $\mathrm{N} 2-\mathrm{C} 22-\mathrm{H} 22 \mathrm{~B}$ & 109.4 & $\mathrm{H} 14 \mathrm{~B}-\mathrm{C} 14-\mathrm{H} 14 \mathrm{C}$ & 109.5 \\
\hline $\mathrm{N} 2-\mathrm{C} 22-\mathrm{C} 23$ & $111.14(11)$ & $\mathrm{C} 33-\mathrm{C} 34-\mathrm{H} 34 \mathrm{~A}$ & 109.5 \\
\hline $\mathrm{H} 22 \mathrm{~A}-\mathrm{C} 22-\mathrm{H} 22 \mathrm{~B}$ & 108.0 & $\mathrm{C} 33-\mathrm{C} 34-\mathrm{H} 34 \mathrm{~B}$ & 109.5 \\
\hline $\mathrm{C} 23-\mathrm{C} 22-\mathrm{H} 22 \mathrm{~A}$ & 109.4 & $\mathrm{C} 33-\mathrm{C} 34-\mathrm{H} 34 \mathrm{C}$ & 109.5 \\
\hline $\mathrm{C} 23-\mathrm{C} 22-\mathrm{H} 22 \mathrm{~B}$ & 109.4 & $\mathrm{H} 34 \mathrm{~A}-\mathrm{C} 34-\mathrm{H} 34 \mathrm{~B}$ & 109.5 \\
\hline $\mathrm{C} 2-\mathrm{C} 3-\mathrm{H} 3$ & 119.0 & $\mathrm{H} 34 \mathrm{~A}-\mathrm{C} 34-\mathrm{H} 34 \mathrm{C}$ & 109.5 \\
\hline $\mathrm{C} 4-\mathrm{C} 3-\mathrm{H} 3$ & 119.0 & $\mathrm{H} 34 \mathrm{~B}-\mathrm{C} 34-\mathrm{H} 34 \mathrm{C}$ & 109.5 \\
\hline $\mathrm{C} 4-\mathrm{C} 3-\mathrm{C} 2$ & $121.94(13)$ & $\mathrm{C} 11-\mathrm{C} 12-\mathrm{H} 12 \mathrm{~A}$ & 109.5 \\
\hline $\mathrm{C} 4-\mathrm{C} 5-\mathrm{H} 5$ & 118.1 & $\mathrm{C} 11-\mathrm{C} 12-\mathrm{H} 12 \mathrm{~B}$ & 109.5 \\
\hline $\mathrm{C} 6-\mathrm{C} 5-\mathrm{H} 5$ & 118.1 & $\mathrm{C} 11-\mathrm{C} 12-\mathrm{H} 12 \mathrm{C}$ & 109.5 \\
\hline $\mathrm{C} 6-\mathrm{C} 5-\mathrm{C} 4$ & $123.81(13)$ & $\mathrm{H} 12 \mathrm{~A}-\mathrm{C} 12-\mathrm{H} 12 \mathrm{~B}$ & 109.5 \\
\hline $\mathrm{O} 2-\mathrm{C} 24-\mathrm{C} 25$ & $119.56(12)$ & $\mathrm{H} 12 \mathrm{~A}-\mathrm{C} 12-\mathrm{H} 12 \mathrm{C}$ & 109.5 \\
\hline $\mathrm{O} 2-\mathrm{C} 24-\mathrm{C} 23$ & 119.97 (13) & $\mathrm{H} 12 \mathrm{~B}-\mathrm{C} 12-\mathrm{H} 12 \mathrm{C}$ & 109.5 \\
\hline $\mathrm{C} 23-\mathrm{C} 24-\mathrm{C} 25$ & $120.45(13)$ & $\mathrm{C} 7-\mathrm{C} 8-\mathrm{H} 8 \mathrm{~A}$ & 109.5 \\
\hline $\mathrm{O} 1-\mathrm{C} 1-\mathrm{C} 2$ & $121.83(12)$ & $\mathrm{C} 7-\mathrm{C} 8-\mathrm{H} 8 \mathrm{~B}$ & 109.5 \\
\hline $\mathrm{O} 1-\mathrm{C} 1-\mathrm{C} 6$ & $117.81(12)$ & $\mathrm{C} 7-\mathrm{C} 8-\mathrm{H} 8 \mathrm{C}$ & 109.5 \\
\hline $\mathrm{C} 2-\mathrm{C} 1-\mathrm{C} 6$ & $120.33(12)$ & $\mathrm{H} 8 \mathrm{~A}-\mathrm{C} 8-\mathrm{H} 8 \mathrm{~B}$ & 109.5 \\
\hline $\mathrm{C} 24-\mathrm{C} 25-\mathrm{C} 33$ & $121.85(13)$ & $\mathrm{H} 8 \mathrm{~A}-\mathrm{C} 8-\mathrm{H} 8 \mathrm{C}$ & 109.5 \\
\hline $\mathrm{C} 26-\mathrm{C} 25-\mathrm{C} 24$ & $117.26(13)$ & $\mathrm{H} 8 \mathrm{~B}-\mathrm{C} 8-\mathrm{H} 8 \mathrm{C}$ & 109.5 \\
\hline $\mathrm{C} 26-\mathrm{C} 25-\mathrm{C} 33$ & $120.89(13)$ & $\mathrm{C} 33-\mathrm{C} 35-\mathrm{H} 35 \mathrm{~A}$ & 109.5 \\
\hline $\mathrm{C} 3-\mathrm{C} 2-\mathrm{C} 15$ & $116.27(12)$ & $\mathrm{C} 33-\mathrm{C} 35-\mathrm{H} 35 \mathrm{~B}$ & 109.5 \\
\hline $\mathrm{C} 1-\mathrm{C} 2-\mathrm{C} 3$ & $119.70(12)$ & $\mathrm{C} 33-\mathrm{C} 35-\mathrm{H} 35 \mathrm{C}$ & 109.5 \\
\hline $\mathrm{C} 1-\mathrm{C} 2-\mathrm{C} 15$ & $123.98(12)$ & $\mathrm{H} 35 \mathrm{~A}-\mathrm{C} 35-\mathrm{H} 35 \mathrm{~B}$ & 109.5 \\
\hline
\end{tabular}




\begin{tabular}{|c|c|}
\hline $\mathrm{C} 25-\mathrm{C} 33-\mathrm{C} 36$ & $111.60(12)$ \\
\hline $\mathrm{C} 34-\mathrm{C} 33-\mathrm{C} 25$ & $111.87(12)$ \\
\hline $\mathrm{C} 34-\mathrm{C} 33-\mathrm{C} 36$ & $107.23(13)$ \\
\hline $\mathrm{C} 35-\mathrm{C} 33-\mathrm{C} 25$ & $109.93(12)$ \\
\hline $\mathrm{C} 35-\mathrm{C} 33-\mathrm{C} 36$ & $108.59(13)$ \\
\hline $\mathrm{C} 35-\mathrm{C} 33-\mathrm{C} 34$ & $107.46(13)$ \\
\hline $\mathrm{C} 3-\mathrm{C} 4-\mathrm{C} 5$ & $116.99(12)$ \\
\hline $\mathrm{C} 3-\mathrm{C} 4-\mathrm{C} 11$ & $122.44(12)$ \\
\hline $\mathrm{C} 5-\mathrm{C} 4-\mathrm{C} 11$ & $120.57(12)$ \\
\hline $\mathrm{C} 25-\mathrm{C} 26-\mathrm{H} 26$ & 118.2 \\
\hline $\mathrm{C} 27-\mathrm{C} 26-\mathrm{C} 25$ & $123.69(13)$ \\
\hline $\mathrm{C} 27-\mathrm{C} 26-\mathrm{H} 26$ & 118.2 \\
\hline $\mathrm{C} 24-\mathrm{C} 23-\mathrm{C} 22$ & $120.24(13)$ \\
\hline $\mathrm{C} 28-\mathrm{C} 23-\mathrm{C} 22$ & $120.05(13)$ \\
\hline $\mathrm{C} 28-\mathrm{C} 23-\mathrm{C} 24$ & $119.62(13)$ \\
\hline $\mathrm{H} 9 \mathrm{~A}-\mathrm{C} 9-\mathrm{H} 9 \mathrm{~B}$ & 109.5 \\
\hline $\mathrm{H} 9 \mathrm{~A}-\mathrm{C} 9-\mathrm{H} 9 \mathrm{C}$ & 109.5 \\
\hline $\mathrm{H} 9 \mathrm{~B}-\mathrm{C} 9-\mathrm{H} 9 \mathrm{C}$ & 109.5 \\
\hline C7-C9-H9A & 109.5 \\
\hline $\mathrm{C} 7-\mathrm{C} 9-\mathrm{H} 9 \mathrm{~B}$ & 109.5 \\
\hline $\mathrm{C} 7-\mathrm{C} 9-\mathrm{H} 9 \mathrm{C}$ & 109.5 \\
\hline $\mathrm{C} 5-\mathrm{C} 6-\mathrm{C} 1$ & $117.17(12)$ \\
\hline $\mathrm{C} 5-\mathrm{C} 6-\mathrm{C} 7$ & $121.74(12)$ \\
\hline $\mathrm{C} 1-\mathrm{C} 6-\mathrm{C} 7$ & $121.09(12)$ \\
\hline $\mathrm{N} 1-\mathrm{C} 15-\mathrm{C} 2$ & $113.51(11)$ \\
\hline $\mathrm{N} 1-\mathrm{C} 15-\mathrm{H} 15 \mathrm{~A}$ & 108.9 \\
\hline $\mathrm{N} 1-\mathrm{C} 15-\mathrm{H} 15 \mathrm{~B}$ & 108.9 \\
\hline $\mathrm{C} 2-\mathrm{C} 15-\mathrm{H} 15 \mathrm{~A}$ & 108.9 \\
\hline $\mathrm{C} 2-\mathrm{C} 15-\mathrm{H} 15 \mathrm{~B}$ & 108.9 \\
\hline $\mathrm{H} 15 \mathrm{~A}-\mathrm{C} 15-\mathrm{H} 15 \mathrm{~B}$ & 107.7 \\
\hline $\mathrm{C} 9-\mathrm{C} 7-\mathrm{C} 8$ & $109.99(13)$ \\
\hline $\mathrm{C} 6-\mathrm{C} 7-\mathrm{C} 9$ & $110.08(12)$ \\
\hline $\mathrm{C} 6-\mathrm{C} 7-\mathrm{C} 8$ & $110.57(12)$ \\
\hline $\mathrm{C} 10-\mathrm{C} 7-\mathrm{C} 9$ & $107.85(12)$ \\
\hline $\mathrm{C} 10-\mathrm{C} 7-\mathrm{C} 6$ & $111.63(12)$ \\
\hline $\mathrm{C} 10-\mathrm{C} 7-\mathrm{C} 8$ & $106.62(12)$ \\
\hline $\mathrm{C} 7-\mathrm{C} 10-\mathrm{H} 10 \mathrm{~A}$ & 109.5 \\
\hline $\mathrm{C} 7-\mathrm{C} 10-\mathrm{H} 10 \mathrm{~B}$ & 109.5 \\
\hline $\mathrm{C} 7-\mathrm{C} 10-\mathrm{H} 10 \mathrm{C}$ & 109.5 \\
\hline $\mathrm{H} 10 \mathrm{~A}-\mathrm{C} 10-\mathrm{H} 10 \mathrm{~B}$ & 109.5 \\
\hline $\mathrm{H} 10 \mathrm{~A}-\mathrm{C} 10-\mathrm{H} 10 \mathrm{C}$ & 109.5 \\
\hline $\mathrm{H} 10 \mathrm{~B}-\mathrm{C} 10-\mathrm{H} 10 \mathrm{C}$ & 109.5 \\
\hline $\mathrm{N} 1-\mathrm{C} 16-\mathrm{H} 16$ & 108.7 \\
\hline $\mathrm{N} 1-\mathrm{C} 16-\mathrm{C} 21$ & $112.61(11)$ \\
\hline $\mathrm{N} 1-\mathrm{C} 16-\mathrm{C} 17$ & $106.26(11)$ \\
\hline $\mathrm{C} 21-\mathrm{C} 16-\mathrm{H} 16$ & 108.7 \\
\hline $\mathrm{C} 17-\mathrm{C} 16-\mathrm{H} 16$ & 108.7 \\
\hline $\mathrm{C} 17-\mathrm{C} 16-\mathrm{C} 21$ & $111.73(12)$ \\
\hline
\end{tabular}

\begin{tabular}{|c|c|}
\hline $\mathrm{H} 35 \mathrm{~A}-\mathrm{C} 35-\mathrm{H} 35 \mathrm{C}$ & 109.5 \\
\hline $\mathrm{H} 35 \mathrm{~B}-\mathrm{C} 35-\mathrm{H} 35 \mathrm{C}$ & 109.5 \\
\hline $\mathrm{N} 2-\mathrm{C} 17-\mathrm{C} 16$ & $107.91(12)$ \\
\hline $\mathrm{N} 2-\mathrm{C} 17-\mathrm{H} 17$ & 107.5 \\
\hline $\mathrm{N} 2-\mathrm{C} 17-\mathrm{C} 18$ & $114.02(12)$ \\
\hline $\mathrm{C} 16-\mathrm{C} 17-\mathrm{H} 17$ & 107.5 \\
\hline $\mathrm{C} 16-\mathrm{C} 17-\mathrm{C} 18$ & $112.17(13)$ \\
\hline $\mathrm{C} 18-\mathrm{C} 17-\mathrm{H} 17$ & 107.5 \\
\hline $\mathrm{C} 11-\mathrm{C} 13-\mathrm{H} 13 \mathrm{~A}$ & 109.5 \\
\hline $\mathrm{C} 11-\mathrm{C} 13-\mathrm{H} 13 \mathrm{~B}$ & 109.5 \\
\hline $\mathrm{C} 11-\mathrm{C} 13-\mathrm{H} 13 \mathrm{C}$ & 109.5 \\
\hline $\mathrm{H} 13 \mathrm{~A}-\mathrm{C} 13-\mathrm{H} 13 \mathrm{~B}$ & 109.5 \\
\hline $\mathrm{H} 13 \mathrm{~A}-\mathrm{C} 13-\mathrm{H} 13 \mathrm{C}$ & 109.5 \\
\hline $\mathrm{H} 13 \mathrm{~B}-\mathrm{C} 13-\mathrm{H} 13 \mathrm{C}$ & 109.5 \\
\hline $\mathrm{C} 19-\mathrm{C} 18-\mathrm{H} 18 \mathrm{~A}$ & 109.6 \\
\hline $\mathrm{C} 19-\mathrm{C} 18-\mathrm{H} 18 \mathrm{~B}$ & 109.6 \\
\hline $\mathrm{C} 17-\mathrm{C} 18-\mathrm{C} 19$ & $110.30(12)$ \\
\hline $\mathrm{C} 17-\mathrm{C} 18-\mathrm{H} 18 \mathrm{~A}$ & 109.6 \\
\hline $\mathrm{C} 17-\mathrm{C} 18-\mathrm{H} 18 \mathrm{~B}$ & 109.6 \\
\hline $\mathrm{H} 18 \mathrm{~A}-\mathrm{C} 18-\mathrm{H} 18 \mathrm{~B}$ & 108.1 \\
\hline $\mathrm{C} 30-\mathrm{C} 29-\mathrm{C} 27$ & $110.44(13)$ \\
\hline $\mathrm{C} 32-\mathrm{C} 29-\mathrm{C} 27$ & $112.47(13)$ \\
\hline $\mathrm{C} 32-\mathrm{C} 29-\mathrm{C} 30$ & $107.76(14)$ \\
\hline $\mathrm{C} 31-\mathrm{C} 29-\mathrm{C} 27$ & $108.10(13)$ \\
\hline $\mathrm{C} 31-\mathrm{C} 29-\mathrm{C} 30$ & $109.91(14)$ \\
\hline $\mathrm{C} 31-\mathrm{C} 29-\mathrm{C} 32$ & $108.13(15)$ \\
\hline $\mathrm{C} 29-\mathrm{C} 30-\mathrm{H} 30 \mathrm{~A}$ & 109.5 \\
\hline $\mathrm{C} 29-\mathrm{C} 30-\mathrm{H} 30 \mathrm{~B}$ & 109.5 \\
\hline $\mathrm{C} 29-\mathrm{C} 30-\mathrm{H} 30 \mathrm{C}$ & 109.5 \\
\hline $\mathrm{H} 30 \mathrm{~A}-\mathrm{C} 30-\mathrm{H} 30 \mathrm{~B}$ & 109.5 \\
\hline $\mathrm{H} 30 \mathrm{~A}-\mathrm{C} 30-\mathrm{H} 30 \mathrm{C}$ & 109.5 \\
\hline $\mathrm{H} 30 \mathrm{~B}-\mathrm{C} 30-\mathrm{H} 30 \mathrm{C}$ & 109.5 \\
\hline $\mathrm{O} 5-\mathrm{C} 37-\mathrm{H} 37 \mathrm{~A}$ & 109.5 \\
\hline $\mathrm{O} 5-\mathrm{C} 37-\mathrm{H} 37 \mathrm{~B}$ & 109.5 \\
\hline $\mathrm{O} 5-\mathrm{C} 37-\mathrm{H} 37 \mathrm{C}$ & 109.5 \\
\hline $\mathrm{H} 37 \mathrm{~A}-\mathrm{C} 37-\mathrm{H} 37 \mathrm{~B}$ & 109.5 \\
\hline H37A-C37-H37C & 109.5 \\
\hline $\mathrm{H} 37 \mathrm{~B}-\mathrm{C} 37-\mathrm{H} 37 \mathrm{C}$ & 109.5 \\
\hline $\mathrm{C} 29-\mathrm{C} 32-\mathrm{H} 32 \mathrm{~A}$ & 109.5 \\
\hline $\mathrm{C} 29-\mathrm{C} 32-\mathrm{H} 32 \mathrm{~B}$ & 109.5 \\
\hline $\mathrm{C} 29-\mathrm{C} 32-\mathrm{H} 32 \mathrm{C}$ & 109.5 \\
\hline $\mathrm{H} 32 \mathrm{~A}-\mathrm{C} 32-\mathrm{H} 32 \mathrm{~B}$ & 109.5 \\
\hline $\mathrm{H} 32 \mathrm{~A}-\mathrm{C} 32-\mathrm{H} 32 \mathrm{C}$ & 109.5 \\
\hline $\mathrm{H} 32 \mathrm{~B}-\mathrm{C} 32-\mathrm{H} 32 \mathrm{C}$ & 109.5 \\
\hline $\mathrm{C} 29-\mathrm{C} 31-\mathrm{H} 31 \mathrm{~A}$ & 109.5 \\
\hline $\mathrm{C} 29-\mathrm{C} 31-\mathrm{H} 31 \mathrm{~B}$ & 109.5 \\
\hline $\mathrm{C} 29-\mathrm{C} 31-\mathrm{H} 31 \mathrm{C}$ & 109.5 \\
\hline $\mathrm{H} 31 \mathrm{~A}-\mathrm{C} 31-\mathrm{H} 31 \mathrm{~B}$ & 109.5 \\
\hline
\end{tabular}




\begin{tabular}{|c|c|c|c|}
\hline $\mathrm{C} 16-\mathrm{C} 21-\mathrm{H} 21 \mathrm{~A}$ & 109.3 & $\mathrm{H} 31 \mathrm{~A}-\mathrm{C} 31-\mathrm{H} 31 \mathrm{C}$ & 109.5 \\
\hline $\mathrm{C} 16-\mathrm{C} 21-\mathrm{H} 21 \mathrm{~B}$ & 109.3 & $\mathrm{H} 31 \mathrm{~B}-\mathrm{C} 31-\mathrm{H} 31 \mathrm{C}$ & 109.5 \\
\hline $\mathrm{C} 16-\mathrm{C} 21-\mathrm{C} 20$ & $111.54(12)$ & $\mathrm{C} 38-\mathrm{O} 0 \mathrm{AA}-\mathrm{H} 0 \mathrm{AA}$ & 109.5 \\
\hline $\mathrm{H} 21 \mathrm{~A}-\mathrm{C} 21-\mathrm{H} 21 \mathrm{~B}$ & 108.0 & $\mathrm{O} 0 \mathrm{AA}-\mathrm{C} 38-\mathrm{H} 38 \mathrm{~A}$ & 109.5 \\
\hline $\mathrm{C} 20-\mathrm{C} 21-\mathrm{H} 21 \mathrm{~A}$ & 109.3 & $\mathrm{O} 0 \mathrm{AA}-\mathrm{C} 38-\mathrm{H} 38 \mathrm{~B}$ & 109.5 \\
\hline $\mathrm{C} 20-\mathrm{C} 21-\mathrm{H} 21 \mathrm{~B}$ & 109.3 & $\mathrm{O} 0 \mathrm{AA}-\mathrm{C} 38-\mathrm{H} 38 \mathrm{C}$ & 109.5 \\
\hline $\mathrm{C} 26-\mathrm{C} 27-\mathrm{C} 28$ & $117.04(13)$ & $\mathrm{H} 38 \mathrm{~A}-\mathrm{C} 38-\mathrm{H} 38 \mathrm{~B}$ & 109.5 \\
\hline $\mathrm{C} 26-\mathrm{C} 27-\mathrm{C} 29$ & $122.83(14)$ & $\mathrm{H} 38 \mathrm{~A}-\mathrm{C} 38-\mathrm{H} 38 \mathrm{C}$ & 109.5 \\
\hline $\mathrm{C} 28-\mathrm{C} 27-\mathrm{C} 29$ & $120.13(13)$ & $\mathrm{H} 38 \mathrm{~B}-\mathrm{C} 38-\mathrm{H} 38 \mathrm{C}$ & 109.5 \\
\hline $\mathrm{C} 23-\mathrm{C} 28-\mathrm{C} 27$ & $121.89(14)$ & & \\
\hline $\mathrm{Mo} 01-\mathrm{O} 1-\mathrm{C} 1-\mathrm{C} 2$ & 31.67 (19) & $\mathrm{C} 1-\mathrm{C} 6-\mathrm{C} 7-\mathrm{C} 10$ & $-177.16(13)$ \\
\hline $\mathrm{Mo} 01-\mathrm{O} 1-\mathrm{C} 1-\mathrm{C} 6$ & $-150.18(10)$ & $\mathrm{C} 1-\mathrm{C} 6-\mathrm{C} 7-\mathrm{C} 8$ & $-58.64(17)$ \\
\hline $\mathrm{Mo} 01-\mathrm{O} 2-\mathrm{C} 24-\mathrm{C} 25$ & $158.74(11)$ & $\mathrm{C} 25-\mathrm{C} 24-\mathrm{C} 23-\mathrm{C} 22$ & $-174.72(13)$ \\
\hline $\mathrm{Mo} 01-\mathrm{O} 2-\mathrm{C} 24-\mathrm{C} 23$ & $-19.8(2)$ & $\mathrm{C} 25-\mathrm{C} 24-\mathrm{C} 23-\mathrm{C} 28$ & $1.8(2)$ \\
\hline $\mathrm{Mo} 01-\mathrm{N} 2-\mathrm{C} 22-\mathrm{C} 23$ & $-73.12(13)$ & $\mathrm{C} 25-\mathrm{C} 26-\mathrm{C} 27-\mathrm{C} 28$ & $0.2(2)$ \\
\hline $\mathrm{Mo} 01-\mathrm{N} 2-\mathrm{C} 17-\mathrm{C} 16$ & $-38.13(13)$ & $\mathrm{C} 25-\mathrm{C} 26-\mathrm{C} 27-\mathrm{C} 29$ & $-179.20(14)$ \\
\hline $\mathrm{Mo} 01-\mathrm{N} 2-\mathrm{C} 17-\mathrm{C} 18$ & $-163.45(10)$ & $\mathrm{C} 2-\mathrm{C} 3-\mathrm{C} 4-\mathrm{C} 5$ & $-2.2(2)$ \\
\hline $\mathrm{Mo} 01-\mathrm{N} 1-\mathrm{C} 15-\mathrm{C} 2$ & $-61.41(13)$ & $\mathrm{C} 2-\mathrm{C} 3-\mathrm{C} 4-\mathrm{C} 11$ & $177.34(13)$ \\
\hline $\mathrm{Mo} 01-\mathrm{N} 1-\mathrm{C} 16-\mathrm{C} 21$ & $-172.68(9)$ & $\mathrm{C} 2-\mathrm{C} 1-\mathrm{C} 6-\mathrm{C} 5$ & $-2.0(2)$ \\
\hline $\mathrm{Mo} 01-\mathrm{N} 1-\mathrm{C} 16-\mathrm{C} 17$ & $-50.05(12)$ & $\mathrm{C} 2-\mathrm{C} 1-\mathrm{C} 6-\mathrm{C} 7$ & $178.87(13)$ \\
\hline $\mathrm{O} 1-\mathrm{C} 1-\mathrm{C} 2-\mathrm{C} 3$ & $178.24(12)$ & $\mathrm{C} 33-\mathrm{C} 25-\mathrm{C} 26-\mathrm{C} 27$ & $-179.11(14)$ \\
\hline $\mathrm{O} 1-\mathrm{C} 1-\mathrm{C} 2-\mathrm{C} 15$ & $-4.4(2)$ & $\mathrm{C} 4-\mathrm{C} 3-\mathrm{C} 2-\mathrm{C} 1$ & $2.1(2)$ \\
\hline $\mathrm{O} 1-\mathrm{C} 1-\mathrm{C} 6-\mathrm{C} 5$ & $179.79(12)$ & $\mathrm{C} 4-\mathrm{C} 3-\mathrm{C} 2-\mathrm{C} 15$ & $-175.52(13)$ \\
\hline $\mathrm{O} 1-\mathrm{C} 1-\mathrm{C} 6-\mathrm{C} 7$ & $0.69(19)$ & $\mathrm{C} 4-\mathrm{C} 5-\mathrm{C} 6-\mathrm{C} 1$ & $1.9(2)$ \\
\hline $\mathrm{O} 2-\mathrm{C} 24-\mathrm{C} 25-\mathrm{C} 33$ & $-0.4(2)$ & $\mathrm{C} 4-\mathrm{C} 5-\mathrm{C} 6-\mathrm{C} 7$ & $-178.97(13)$ \\
\hline $\mathrm{O} 2-\mathrm{C} 24-\mathrm{C} 25-\mathrm{C} 26$ & $178.78(13)$ & $\mathrm{C} 26-\mathrm{C} 25-\mathrm{C} 33-\mathrm{C} 36$ & $127.48(15)$ \\
\hline $\mathrm{O} 2-\mathrm{C} 24-\mathrm{C} 23-\mathrm{C} 22$ & $3.8(2)$ & $\mathrm{C} 26-\mathrm{C} 25-\mathrm{C} 33-\mathrm{C} 34$ & $7.33(19)$ \\
\hline $\mathrm{O} 2-\mathrm{C} 24-\mathrm{C} 23-\mathrm{C} 28$ & $-179.71(13)$ & $\mathrm{C} 26-\mathrm{C} 25-\mathrm{C} 33-\mathrm{C} 35$ & $-111.97(16)$ \\
\hline $\mathrm{N} 2-\mathrm{C} 22-\mathrm{C} 23-\mathrm{C} 24$ & $45.68(19)$ & $\mathrm{C} 26-\mathrm{C} 27-\mathrm{C} 28-\mathrm{C} 23$ & $-1.3(2)$ \\
\hline $\mathrm{N} 2-\mathrm{C} 22-\mathrm{C} 23-\mathrm{C} 28$ & $-130.82(14)$ & $\mathrm{C} 26-\mathrm{C} 27-\mathrm{C} 29-\mathrm{C} 30$ & $-126.65(16)$ \\
\hline $\mathrm{N} 2-\mathrm{C} 17-\mathrm{C} 18-\mathrm{C} 19$ & $178.60(13)$ & $\mathrm{C} 26-\mathrm{C} 27-\mathrm{C} 29-\mathrm{C} 32$ & $-6.2(2)$ \\
\hline $\mathrm{N} 1-\mathrm{C} 16-\mathrm{C} 21-\mathrm{C} 20$ & $172.29(13)$ & $\mathrm{C} 26-\mathrm{C} 27-\mathrm{C} 29-\mathrm{C} 31$ & $113.07(17)$ \\
\hline $\mathrm{N} 1-\mathrm{C} 16-\mathrm{C} 17-\mathrm{N} 2$ & $56.94(14)$ & $\mathrm{C} 23-\mathrm{C} 24-\mathrm{C} 25-\mathrm{C} 33$ & $178.13(13)$ \\
\hline $\mathrm{N} 1-\mathrm{C} 16-\mathrm{C} 17-\mathrm{C} 18$ & $-176.64(12)$ & $\mathrm{C} 23-\mathrm{C} 24-\mathrm{C} 25-\mathrm{C} 26$ & $-2.7(2)$ \\
\hline $\mathrm{C} 22-\mathrm{N} 2-\mathrm{C} 17-\mathrm{C} 16$ & $-163.01(11)$ & $\mathrm{C} 6-\mathrm{C} 5-\mathrm{C} 4-\mathrm{C} 3$ & $0.1(2)$ \\
\hline $\mathrm{C} 22-\mathrm{N} 2-\mathrm{C} 17-\mathrm{C} 18$ & $71.67(16)$ & $\mathrm{C} 6-\mathrm{C} 5-\mathrm{C} 4-\mathrm{C} 11$ & $-179.40(13)$ \\
\hline $\mathrm{C} 22-\mathrm{C} 23-\mathrm{C} 28-\mathrm{C} 27$ & $176.79(14)$ & $\mathrm{C} 6-\mathrm{C} 1-\mathrm{C} 2-\mathrm{C} 3$ & $0.1(2)$ \\
\hline $\mathrm{C} 3-\mathrm{C} 2-\mathrm{C} 15-\mathrm{N} 1$ & $-154.63(12)$ & $\mathrm{C} 6-\mathrm{C} 1-\mathrm{C} 2-\mathrm{C} 15$ & $177.52(13)$ \\
\hline $\mathrm{C} 3-\mathrm{C} 4-\mathrm{C} 11-\mathrm{C} 14$ & $-114.88(15)$ & $\mathrm{C} 15-\mathrm{N} 1-\mathrm{C} 16-\mathrm{C} 21$ & $62.12(15)$ \\
\hline $\mathrm{C} 3-\mathrm{C} 4-\mathrm{C} 11-\mathrm{C} 12$ & $5.36(19)$ & $\mathrm{C} 15-\mathrm{N} 1-\mathrm{C} 16-\mathrm{C} 17$ & $-175.25(11)$ \\
\hline $\mathrm{C} 3-\mathrm{C} 4-\mathrm{C} 11-\mathrm{C} 13$ & $125.68(14)$ & $\mathrm{C} 16-\mathrm{N} 1-\mathrm{C} 15-\mathrm{C} 2$ & $64.63(15)$ \\
\hline $\mathrm{C} 5-\mathrm{C} 4-\mathrm{C} 11-\mathrm{C} 14$ & $64.61(17)$ & $\mathrm{C} 16-\mathrm{C} 21-\mathrm{C} 20-\mathrm{C} 19$ & $-55.27(18)$ \\
\hline $\mathrm{C} 5-\mathrm{C} 4-\mathrm{C} 11-\mathrm{C} 12$ & $-175.14(13)$ & $\mathrm{C} 16-\mathrm{C} 17-\mathrm{C} 18-\mathrm{C} 19$ & $55.56(18)$ \\
\hline $\mathrm{C} 5-\mathrm{C} 4-\mathrm{C} 11-\mathrm{C} 13$ & $-54.82(17)$ & $\mathrm{C} 21-\mathrm{C} 16-\mathrm{C} 17-\mathrm{N} 2$ & $-179.87(11)$ \\
\hline $\mathrm{C} 5-\mathrm{C} 6-\mathrm{C} 7-\mathrm{C} 9$ & $-115.97(15)$ & $\mathrm{C} 21-\mathrm{C} 16-\mathrm{C} 17-\mathrm{C} 18$ & $-53.46(17)$ \\
\hline $\mathrm{C} 5-\mathrm{C} 6-\mathrm{C} 7-\mathrm{C} 10$ & $3.78(19)$ & $\mathrm{C} 28-\mathrm{C} 27-\mathrm{C} 29-\mathrm{C} 30$ & $53.95(19)$ \\
\hline $\mathrm{C} 5-\mathrm{C} 6-\mathrm{C} 7-\mathrm{C} 8$ & $122.30(14)$ & $\mathrm{C} 28-\mathrm{C} 27-\mathrm{C} 29-\mathrm{C} 32$ & $174.37(15)$ \\
\hline
\end{tabular}




$\begin{array}{llll}\mathrm{C} 24-\mathrm{C} 25-\mathrm{C} 33-\mathrm{C} 36 & -53.41(18) & \mathrm{C} 28-\mathrm{C} 27-\mathrm{C} 29-\mathrm{C} 31 & -66.33(19) \\ \mathrm{C} 24-\mathrm{C} 25-\mathrm{C} 33-\mathrm{C} 34 & -173.56(13) & \mathrm{C} 20-\mathrm{C} 19-\mathrm{C} 18-\mathrm{C} 17 & -57.38(18) \\ \mathrm{C} 24-\mathrm{C} 25-\mathrm{C} 33-\mathrm{C} 35 & 67.14(17) & \mathrm{C} 17-\mathrm{N} 2-\mathrm{C} 22-\mathrm{C} 23 & 54.25(15) \\ \mathrm{C} 24-\mathrm{C} 25-\mathrm{C} 26-\mathrm{C} 27 & 1.7(2) & \mathrm{C} 17-\mathrm{C} 16-\mathrm{C} 21-\mathrm{C} 20 & 52.79(17) \\ \mathrm{C} 24-\mathrm{C} 23-\mathrm{C} 28-\mathrm{C} 27 & 0.3(2) & \mathrm{C} 18-\mathrm{C} 19-\mathrm{C} 20-\mathrm{C} 21 & 57.58(18) \\ \mathrm{C} 1-\mathrm{C} 2-\mathrm{C} 15-\mathrm{N} 1 & 27.90(19) & \mathrm{C} 29-\mathrm{C} 27-\mathrm{C} 28-\mathrm{C} 23 & 178.18(14) \\ \mathrm{C} 1-\mathrm{C} 6-\mathrm{C} 7-\mathrm{C} 9 & 63.09(17) & & \end{array}$

Hydrogen-bond geometry $\left(\AA,{ }^{\circ}\right)$

\begin{tabular}{lllll}
\hline$D-\mathrm{H}^{\cdots} \cdots A$ & $D-\mathrm{H}$ & $\mathrm{H} \cdots A$ & $D \cdots A$ & $D-\mathrm{H}^{\cdots} \cdots A$ \\
\hline $\mathrm{N} 2-\mathrm{H} 2 \cdots \mathrm{O} 5^{\mathrm{i}}$ & 1.00 & 2.00 & $2.9319(16)$ & 153 \\
$\mathrm{O} 5-\mathrm{H} 5 A \cdots \mathrm{O} 4$ & 0.84 & 1.94 & $2.7837(16)$ & 177 \\
\hline
\end{tabular}

Symmetry code: (i) $-x+1,-y+1,-z+1$.

Dioxido\{2,2'-[l,2-phenylenebis(iminomethylene)]bis(phenolato)\}molybdenum(VI) dimethylformamide disolvate (1b)

\section{Crystal data}

$\left[\mathrm{Mo}\left(\mathrm{C}_{20} \mathrm{H}_{18} \mathrm{~N}_{2} \mathrm{O}_{2}\right) \mathrm{O}_{2}\right] \cdot 2 \mathrm{C}_{3} \mathrm{H}_{7} \mathrm{NO}$

$M_{r}=592.49$

Triclinic, $P \overline{1}$

$a=9.601 \AA$

$b=12.860 \AA$

$c=21.428 \AA$

$\alpha=91.44^{\circ}$

$\beta=91.49^{\circ}$

$\gamma=93.22^{\circ}$

$V=2639.8 \AA^{3}$

Data collection

Bruker APEXII CCD diffractometer

$\varphi$ and $\omega$ scans

Absorption correction: multi-scan

(SADABS; Bruker, 2016)

$T_{\min }=0.664, T_{\max }=0.737$

146655 measured reflections

\section{Refinement}

Refinement on $F^{2}$

Least-squares matrix: full

$R\left[F^{2}>2 \sigma\left(F^{2}\right)\right]=0.035$

$w R\left(F^{2}\right)=0.065$

$S=1.06$

7625 reflections

683 parameters

0 restraints

Primary atom site location: dual
$Z=4$

$F(000)=1224$

$D_{\mathrm{x}}=1.491 \mathrm{Mg} \mathrm{m}^{-3}$

Mo $K \alpha$ radiation, $\lambda=0.71073 \AA$

Cell parameters from 9515 reflections

$\theta=2.3-49.3^{\circ}$

$\mu=0.54 \mathrm{~mm}^{-1}$

$T=100 \mathrm{~K}$

Plate, yellow

$0.34 \times 0.29 \times 0.29 \mathrm{~mm}$

7625 independent reflections 6364 reflections with $I>2 \sigma(I)$

$R_{\text {int }}=0.056$

$\theta_{\max }=27.1^{\circ}, \theta_{\min }=2.7^{\circ}$

$h=-12 \rightarrow 12$

$k=-16 \rightarrow 16$

$l=-27 \rightarrow 27$

Hydrogen site location: mixed

$\mathrm{H}$ atoms treated by a mixture of independent and constrained refinement

$w=1 /\left[\sigma^{2}\left(F_{\mathrm{o}}{ }^{2}\right)+(0.0214 P)^{2}+0.638 P\right]$

where $P=\left(F_{\mathrm{o}}^{2}+2 F_{\mathrm{c}}^{2}\right) / 3$

$(\Delta / \sigma)_{\max }=0.002$

$\Delta \rho_{\max }=0.35 \mathrm{e} \AA^{-3}$

$\Delta \rho_{\min }=-0.38$ e $\AA^{-3}$ 


\section{Special details}

Geometry. All esds (except the esd in the dihedral angle between two 1.s. planes) are estimated using the full covariance matrix. The cell esds are taken into account individually in the estimation of esds in distances, angles and torsion angles; correlations between esds in cell parameters are only used when they are defined by crystal symmetry. An approximate (isotropic) treatment of cell esds is used for estimating esds involving l.s. planes.

Fractional atomic coordinates and isotropic or equivalent isotropic displacement parameters $\left(\AA^{2}\right)$

\begin{tabular}{|c|c|c|c|c|}
\hline & $x$ & $y$ & $z$ & $U_{\text {iso }} * / U_{\text {eq }}$ \\
\hline Mo01 & $0.90908(2)$ & $0.88143(2)$ & $0.23791(2)$ & $0.01047(6)$ \\
\hline Mo02 & $0.40906(2)$ & $0.38140(2)$ & $0.26211(2)$ & $0.01045(6)$ \\
\hline O5 & $0.36926(14)$ & $0.42698(12)$ & $0.17689(7)$ & $0.0135(3)$ \\
\hline $\mathrm{O} 1$ & 0.86930 (14) & $0.92696(12)$ & $0.32311(7)$ & $0.0134(3)$ \\
\hline $\mathrm{O} 3$ & $1.00613(14)$ & $0.98497(13)$ & $0.21447(8)$ & $0.0165(3)$ \\
\hline O8 & $0.51765(15)$ & $0.28410(13)$ & $0.23806(8)$ & $0.0152(3)$ \\
\hline O7 & $0.50605(15)$ & $0.48499(13)$ & $0.28555(8)$ & $0.0169(3)$ \\
\hline $\mathrm{N} 2$ & $0.72468(16)$ & $0.77486(14)$ & $0.26078(8)$ & $0.0098(3)$ \\
\hline H008 & 0.736567 & 0.755680 & 0.305460 & $0.012 *$ \\
\hline N4 & $0.22457(16)$ & $0.27483(14)$ & $0.23925(9)$ & $0.0103(4)$ \\
\hline H009 & 0.231199 & 0.252423 & 0.194450 & $0.012 *$ \\
\hline $\mathrm{O} 4$ & $1.01781(15)$ & $0.78420(13)$ & $0.26191(8)$ & $0.0152(3)$ \\
\hline $\mathrm{O} 2$ & $0.87093(14)$ & $0.83127(13)$ & $0.15364(7)$ & $0.0141(3)$ \\
\hline O6 & $0.37087(14)$ & $0.33128(13)$ & $0.34638(7)$ & $0.0141(3)$ \\
\hline N1 & $0.71375(17)$ & $0.97852(14)$ & $0.21773(9)$ & $0.0101(3)$ \\
\hline N3 & $0.21399(17)$ & $0.47869(14)$ & $0.28219(9)$ & $0.0101(3)$ \\
\hline O9 & $0.2105(3)$ & $0.59956(16)$ & $0.39734(9)$ & $0.0395(5)$ \\
\hline $\mathrm{O} 10$ & $0.29034(18)$ & $0.15986(15)$ & $0.12529(8)$ & $0.0235(4)$ \\
\hline O11 & $0.79013(18)$ & $0.65963(14)$ & $0.37463(8)$ & $0.0234(4)$ \\
\hline $\mathrm{O} 12$ & $0.7110(3)$ & $1.09959(16)$ & $0.10270(9)$ & $0.0388(5)$ \\
\hline $\mathrm{C} 40$ & $0.2694(2)$ & $0.27959(17)$ & $0.37595(10)$ & $0.0133(4)$ \\
\hline $\mathrm{C} 33$ & $0.09199(19)$ & $0.32526(16)$ & $0.24527(10)$ & 0.0097 (4) \\
\hline N6 & $0.2294(2)$ & $0.00745(18)$ & $0.07856(10)$ & $0.0229(4)$ \\
\hline $\mathrm{C} 13$ & $0.59214(19)$ & $0.82531(17)$ & $0.25477(10)$ & $0.0100(4)$ \\
\hline N7 & $0.7296(2)$ & $0.50764(18)$ & $0.42150(10)$ & $0.0231(5)$ \\
\hline $\mathrm{C} 2$ & $0.6928(2)$ & $1.05716(17)$ & $0.32295(11)$ & $0.0132(4)$ \\
\hline $\mathrm{C} 44$ & $0.2062(2)$ & $0.0933(2)$ & $0.11184(11)$ & $0.0201(5)$ \\
\hline H00P & 0.114108 & 0.101089 & 0.125731 & $0.024 *$ \\
\hline $\mathrm{C} 20$ & $0.7691(2)$ & $0.77942(17)$ & $0.12402(10)$ & 0.0138 (4) \\
\hline $\mathrm{C} 26$ & $0.1928(2)$ & $0.55726(17)$ & $0.17703(11)$ & $0.0133(4)$ \\
\hline $\mathrm{C} 35$ & $0.1970(2)$ & $0.20156(17)$ & $0.34516(11)$ & $0.0138(4)$ \\
\hline $\mathrm{C} 32$ & $-0.0270(2)$ & $0.27625(17)$ & $0.22922(10)$ & $0.0124(4)$ \\
\hline $\mathrm{H} 00 \mathrm{~T}$ & -0.028382 & 0.209159 & 0.209538 & $0.015^{*}$ \\
\hline $\mathrm{C} 21$ & $0.2697(2)$ & $0.48024(18)$ & $0.14646(11)$ & $0.0140(4)$ \\
\hline N5 & $0.2261(2)$ & $0.77472(17)$ & $0.40850(10)$ & $0.0223(4)$ \\
\hline $\mathrm{C} 47$ & $0.7060(2)$ & $0.5934(2)$ & $0.38822(11)$ & $0.0196(5)$ \\
\hline H00W & 0.612568 & 0.601350 & 0.374316 & $0.024 *$ \\
\hline N8 & $0.7263(2)$ & $1.27474(17)$ & $0.09144(10)$ & $0.0223(4)$ \\
\hline $\mathrm{C} 12$ & $0.4730(2)$ & $0.77623(17)$ & $0.27093(10)$ & $0.0125(4)$ \\
\hline
\end{tabular}




\begin{tabular}{|c|c|c|c|c|}
\hline H0OY & 0.473625 & 0.710632 & 0.290319 & $0.015^{*}$ \\
\hline C39 & $0.2388(2)$ & $0.3050(2)$ & 0.43809 (11) & $0.0201(5)$ \\
\hline H00Z & 0.291477 & 0.360886 & 0.458964 & $0.024 *$ \\
\hline $\mathrm{C} 8$ & 0.58707 (19) & $0.92488(16)$ & $0.22796(10)$ & $0.0091(4)$ \\
\hline $\mathrm{C} 29$ & $-0.0390(2)$ & $0.47127(17)$ & $0.28501(10)$ & $0.0123(4)$ \\
\hline H011 & -0.037498 & 0.538591 & 0.304393 & $0.015^{*}$ \\
\hline C19 & $0.7389(2)$ & $0.8050(2)$ & $0.06185(12)$ & $0.0203(5)$ \\
\hline H012 & 0.791590 & 0.859381 & 0.042563 & $0.024 *$ \\
\hline $\mathrm{C} 15$ & $0.6965(2)$ & $0.70132(17)$ & 0.15474 (11) & 0.0142 \\
\hline $\mathrm{C} 28$ & $0.08690(19)$ & $0.42482(17)$ & $0.27184(10)$ & $0.0091(4)$ \\
\hline $\mathrm{C} 41$ & $0.1618(3)$ & $0.6874(2)$ & $0.39361(12)$ & $0.0283(6)$ \\
\hline H015 & 0.068263 & 0.689541 & 0.378304 & $0.034 *$ \\
\hline $\mathrm{C} 9$ & $0.4611(2)$ & $0.97132(17)$ & $0.21493(10)$ & $0.0125(4)$ \\
\hline H016 & 0.460785 & 1.037336 & 0.196026 & $0.015^{*}$ \\
\hline $\mathrm{C} 1$ & $0.7697(2)$ & $0.98025(18)$ & $0.35362(11)$ & $0.0138(4)$ \\
\hline $\mathrm{C} 5$ & $0.6467(3)$ & $1.0132(2)$ & $0.44922(12)$ & $0.0256(6)$ \\
\hline H018 & 0.630612 & 0.998654 & 0.491758 & $0.031 *$ \\
\hline C34 & $0.2286(2)$ & 0.17687 (17) & $0.27776(11)$ & $0.0134(4)$ \\
\hline $\mathrm{H} 01 \mathrm{G}$ & 0.158999 & 0.123370 & 0.260434 & $0.016^{*}$ \\
\hline $\mathrm{H} 01 \mathrm{H}$ & 0.322041 & 0.148400 & 0.275457 & $0.016^{*}$ \\
\hline $\mathrm{C} 23$ & $0.1469(3)$ & $0.5132(2)$ & $0.05077(12)$ & $0.0258(6)$ \\
\hline H01I & 0.130229 & 0.498464 & 0.007471 & $0.031 *$ \\
\hline C6 & $0.7459(2)$ & $0.9580(2)$ & $0.41654(12)$ & $0.0204(5)$ \\
\hline H01B & 0.796107 & 0.906242 & 0.436774 & $0.024 *$ \\
\hline $\mathrm{C} 50$ & $0.6613(3)$ & $1.1871(2)$ & $0.10638(12)$ & $0.0282(6)$ \\
\hline H3AA & 0.569131 & 1.190364 & 0.121161 & $0.034^{*}$ \\
\hline $\mathrm{C} 25$ & $0.0929(2)$ & $0.61129(19)$ & $0.14338(11)$ & $0.0189(5)$ \\
\hline H01J & 0.041127 & 0.662013 & 0.164100 & $0.023 *$ \\
\hline $\mathrm{C} 52$ & $0.6615(3)$ & $1.3763(2)$ & 0.09717 (13) & $0.0289(6)$ \\
\hline H4AA & 0.655244 & 1.407393 & 0.055983 & $0.043^{*}$ \\
\hline $\mathrm{H}$ & 0.718693 & 1.422943 & 0.125606 & $0.043 *$ \\
\hline HA & 0.567704 & 1.365643 & 0.113700 & $0.043 *$ \\
\hline $\mathrm{C} 3$ & $0.5933(2)$ & $1.11153(19)$ & $0.35649(12)$ & $0.0194(5)$ \\
\hline $\mathrm{H} 01 \mathrm{~F}$ & 0.541726 & 1.162479 & 0.336215 & $0.023 *$ \\
\hline C36 & 0.0928 (2) & $0.1508(2)$ & $0.37725(12)$ & $0.0227(5)$ \\
\hline $\mathrm{H} 01 \mathrm{~K}$ & 0.038307 & 0.096025 & 0.356264 & $0.027 *$ \\
\hline $\mathrm{C} 43$ & 0.1617 (3) & $0.8762(2)$ & $0.40289(13)$ & $0.0286(6)$ \\
\hline H01V & 0.066608 & 0.864836 & 0.385335 & $0.043^{*}$ \\
\hline H01X & 0.217185 & 0.920818 & 0.375361 & $0.043^{*}$ \\
\hline H01 & 0.158583 & 0.910086 & 0.444276 & $0.043^{*}$ \\
\hline C14 & $0.7286(2)$ & 0.67698 (17) & $0.22211(11)$ & $0.0132(4)$ \\
\hline H01A & 0.658777 & 0.624167 & 0.236808 & $0.016^{*}$ \\
\hline $\mathrm{H} 01 \mathrm{C}$ & 0.822034 & 0.648551 & 0.225945 & $0.016^{*}$ \\
\hline $\mathrm{C} 7$ & $0.7171(2)$ & 1.07799 (17) & $0.25468(11)$ & $0.0132(4)$ \\
\hline H01D & 0.643980 & 1.122212 & 0.238363 & $0.016^{*}$ \\
\hline H01E & 0.808727 & 1.116005 & 0.250437 & $0.016^{*}$ \\
\hline $\mathrm{C} 27$ & $0.2171(2)$ & $0.57802(17)$ & $0.24528(11)$ & $0.0134(4)$ \\
\hline H01L & 0.144556 & 0.623135 & 0.260818 & $0.016^{*}$ \\
\hline
\end{tabular}




\begin{tabular}{|c|c|c|c|c|}
\hline H01M & 0.308883 & 0.616118 & 0.252287 & $0.016^{*}$ \\
\hline $\mathrm{C} 22$ & $0.2461(2)$ & $0.4580(2)$ & $0.08324(11)$ & $0.0201(5)$ \\
\hline H01Q & 0.296160 & 0.406416 & 0.062409 & $0.024^{*}$ \\
\hline C38 & $0.1364(3)$ & $0.2525(2)$ & $0.46952(12)$ & $0.0268(6)$ \\
\hline $\mathrm{H} 01 \mathrm{~S}$ & 0.118153 & 0.270242 & 0.511713 & $0.032 *$ \\
\hline C18 & $0.6362(3)$ & $0.7523(2)$ & $0.03063(12)$ & $0.0273(6)$ \\
\hline $\mathrm{H} 01 \mathrm{~N}$ & 0.614082 & 0.767239 & -0.011561 & $0.033^{*}$ \\
\hline C16 & $0.5928(2)$ & $0.6508(2)$ & $0.12279(12)$ & $0.0221(5)$ \\
\hline $\mathrm{H} 01 \mathrm{O}$ & 0.538427 & 0.597338 & 0.142117 & $0.027 *$ \\
\hline $\mathrm{C} 17$ & $0.5624(3)$ & $0.6753(2)$ & $0.06102(13)$ & $0.0294(6)$ \\
\hline $\mathrm{H} 01 \mathrm{P}$ & 0.487821 & 0.637301 & 0.039190 & $0.035^{*}$ \\
\hline C37 & $0.0627(3)$ & $0.1754(2)$ & 0.43895 (13) & $0.0293(6)$ \\
\hline H01T & -0.010064 & 0.137342 & 0.459158 & $0.035^{*}$ \\
\hline $\mathrm{C} 4$ & $0.5703(3)$ & 1.0908 (2) & $0.41929(12)$ & $0.0278(6)$ \\
\hline H01R & 0.504234 & 1.127999 & 0.441810 & $0.033^{*}$ \\
\hline C46 & $0.3651(3)$ & $-0.0174(2)$ & 0.05437 (13) & $0.0305(6)$ \\
\hline H1AA & 0.357558 & -0.026473 & 0.008834 & $0.046^{*}$ \\
\hline HB & 0.394488 & -0.082007 & 0.072697 & $0.046^{*}$ \\
\hline $\mathrm{HC}$ & 0.434179 & 0.039525 & 0.065371 & $0.046^{*}$ \\
\hline $\mathrm{C} 24$ & $0.0704(3)$ & $0.5906(2)$ & 0.08077 (12) & $0.0271(6)$ \\
\hline H01U & 0.004413 & 0.627654 & 0.057686 & $0.032 *$ \\
\hline $\mathrm{C} 42$ & $0.3670(3)$ & 0.7719 (2) & $0.43037(14)$ & $0.0314(6)$ \\
\hline H0AA & 0.372846 & 0.789365 & 0.475180 & $0.047 *$ \\
\hline HD & 0.425537 & 0.822557 & 0.407903 & $0.047 *$ \\
\hline $\mathrm{HE}$ & 0.399668 & 0.701912 & 0.423092 & $0.047^{*}$ \\
\hline C51 & 0.8669 (3) & $1.2720(2)$ & 0.06964 (14) & $0.0310(6)$ \\
\hline H5AA & 0.902116 & 1.203125 & 0.076874 & $0.046^{*}$ \\
\hline $\mathrm{HF}$ & 0.926724 & 1.325264 & 0.092367 & $0.046^{*}$ \\
\hline $\mathrm{HG}$ & 0.867536 & 1.285745 & 0.024869 & $0.046^{*}$ \\
\hline C11 & $0.3464(2)$ & $0.82277(18)$ & $0.25892(11)$ & $0.0153(4)$ \\
\hline H01W & 0.261648 & 0.787886 & 0.270639 & $0.018^{*}$ \\
\hline C48 & $0.6238(4)$ & $0.4321(3)$ & $0.43236(16)$ & $0.0491(9)$ \\
\hline $\mathrm{H} 2 \mathrm{AA}$ & 0.613110 & 0.425094 & 0.477441 & $0.074^{*}$ \\
\hline $\mathrm{HH}$ & 0.647881 & 0.365190 & 0.413854 & $0.074 *$ \\
\hline HI & 0.535937 & 0.452815 & 0.413397 & $0.074^{*}$ \\
\hline $\mathrm{C} 30$ & $-0.1581(2)$ & $0.42067(18)$ & $0.27025(11)$ & $0.0157(5)$ \\
\hline H01Y & -0.244489 & 0.449657 & 0.279109 & $0.019 *$ \\
\hline C31 & $-0.1535(2)$ & $0.32303(18)$ & $0.24115(11)$ & $0.0156(5)$ \\
\hline H01Z & -0.238872 & 0.286402 & 0.228821 & $0.019 *$ \\
\hline C49 & $0.8649(3)$ & $0.4826(2)$ & $0.44569(13)$ & $0.0300(6)$ \\
\hline H02D & 0.933605 & 0.539377 & 0.437160 & $0.045^{*}$ \\
\hline H02E & 0.892550 & 0.417988 & 0.425468 & $0.045^{*}$ \\
\hline $\mathrm{H} 02 \mathrm{~F}$ & 0.860619 & 0.473340 & 0.490867 & $0.045^{*}$ \\
\hline $\mathrm{C} 45$ & 0.1239 (4) & $-0.0683(3)$ & $0.06749(17)$ & $0.0505(9)$ \\
\hline $\mathrm{H} 02 \mathrm{~A}$ & 0.149070 & -0.133015 & 0.087006 & $0.076^{*}$ \\
\hline H02B & 0.110078 & -0.080595 & 0.022377 & $0.076^{*}$ \\
\hline $\mathrm{H} 02 \mathrm{C}$ & 0.037358 & -0.045270 & 0.085225 & $0.076^{*}$ \\
\hline $\mathrm{C} 10$ & $0.3417(2)$ & $0.92073(18)$ & $0.22967(11)$ & 0.0158 \\
\hline
\end{tabular}




\begin{tabular}{lllll} 
H022 & 0.254780 & 0.949711 & 0.220761 & $0.019^{*}$ \\
H00D & $0.711(3)$ & $0.996(2)$ & $0.1797(14)$ & $0.024(8)^{*}$ \\
H00E & $0.207(3)$ & $0.493(2)$ & $0.3179(14)$ & $0.015(7)^{*}$ \\
\hline
\end{tabular}

Atomic displacement parameters $\left(\AA^{2}\right)$

\begin{tabular}{|c|c|c|c|c|c|c|}
\hline & $U^{11}$ & $U^{22}$ & $U^{33}$ & $U^{12}$ & $U^{13}$ & $U^{23}$ \\
\hline Mo01 & $0.00439(8)$ & $0.00933(10)$ & $0.01750(10)$ & $-0.00061(6)$ & $0.00009(6)$ & $-0.00087(7)$ \\
\hline Mo02 & $0.00428(8)$ & $0.00942(10)$ & $0.01748(10)$ & $-0.00056(6)$ & $-0.00076(6)$ & $0.00006(7)$ \\
\hline $\mathrm{O} 5$ & $0.0091(6)$ & $0.0138(8)$ & $0.0178(8)$ & $0.0011(6)$ & $0.0010(6)$ & $0.0020(6)$ \\
\hline $\mathrm{O} 1$ & $0.0087(6)$ & $0.0138(8)$ & $0.0175(8)$ & $0.0013(6)$ & $-0.0016(6)$ & $-0.0029(6)$ \\
\hline $\mathrm{O} 3$ & $0.0082(6)$ & $0.0153(8)$ & $0.0254(9)$ & $-0.0046(6)$ & $0.0021(6)$ & $-0.0019(7)$ \\
\hline $\mathrm{O} 8$ & $0.0081(6)$ & $0.0142(8)$ & $0.0235(8)$ & $0.0008(6)$ & $0.0000(6)$ & $0.0017(7)$ \\
\hline $\mathrm{O} 7$ & $0.0084(6)$ & $0.0169(8)$ & $0.0248(9)$ & $-0.0037(6)$ & $-0.0035(6)$ & $0.0011(7)$ \\
\hline N2 & $0.0056(7)$ & $0.0088(9)$ & $0.0148(9)$ & $-0.0011(7)$ & $-0.0022(6)$ & $-0.0003(7)$ \\
\hline N4 & $0.0060(7)$ & $0.0090(9)$ & $0.0158(9)$ & $0.0001(7)$ & $0.0017(6)$ & $-0.0022(7)$ \\
\hline $\mathrm{O} 4$ & $0.0076(6)$ & $0.0140(8)$ & $0.0237(8)$ & $0.0006(6)$ & $-0.0014(6)$ & $-0.0030(7)$ \\
\hline $\mathrm{O} 2$ & $0.0093(6)$ & $0.0152(8)$ & $0.0175(8)$ & $-0.0041(6)$ & $0.0021(6)$ & $-0.0007(6)$ \\
\hline O6 & $0.0085(6)$ & $0.0155(8)$ & $0.0175(8)$ & $-0.0043(6)$ & $-0.0038(6)$ & $0.0003(6)$ \\
\hline N1 & $0.0086(7)$ & $0.0100(9)$ & $0.0115(9)$ & $-0.0008(7)$ & $0.0018(6)$ & $0.0010(7)$ \\
\hline N3 & $0.0084(7)$ & $0.0092(9)$ & $0.0123(9)$ & $-0.0013(7)$ & $-0.0025(7)$ & $-0.0028(7)$ \\
\hline O9 & $0.0747(15)$ & $0.0213(10)$ & $0.0206(10)$ & $-0.0087(11)$ & $-0.0054(10)$ & $-0.0046(8)$ \\
\hline $\mathrm{O} 10$ & $0.0253(8)$ & $0.0227(9)$ & $0.0220(9)$ & $-0.0024(8)$ & $0.0047(7)$ & $-0.0052(7)$ \\
\hline O11 & $0.0260(8)$ & $0.0214(9)$ & $0.0221(9)$ & $-0.0029(7)$ & $-0.0064(7)$ & $0.0034(7)$ \\
\hline $\mathrm{O} 12$ & $0.0738(15)$ & $0.0221(10)$ & $0.0194(10)$ & $-0.0091(10)$ & $0.0019(10)$ & $0.0024(8)$ \\
\hline $\mathrm{C} 40$ & $0.0110(8)$ & $0.0126(10)$ & $0.0165(11)$ & $0.0010(8)$ & $-0.0008(8)$ & $0.0035(8)$ \\
\hline $\mathrm{C} 33$ & $0.0077(8)$ & $0.0099(10)$ & $0.0117(10)$ & $0.0008(8)$ & $0.0009(7)$ & $0.0004(8)$ \\
\hline N6 & $0.0258(10)$ & $0.0234(11)$ & $0.0184(11)$ & $-0.0088(9)$ & $0.0065(8)$ & $-0.0060(9)$ \\
\hline $\mathrm{C} 13$ & $0.0089(8)$ & $0.0097(10)$ & $0.0114(10)$ & $0.0014(8)$ & $-0.0011(7)$ & $-0.0011(8)$ \\
\hline N7 & $0.0264(10)$ & $0.0230(11)$ & $0.0185(11)$ & $-0.0091(9)$ & $-0.0071(8)$ & $0.0041(9)$ \\
\hline $\mathrm{C} 2$ & $0.0099(8)$ & $0.0101(10)$ & $0.0189(11)$ & $-0.0033(8)$ & $-0.0023(8)$ & $-0.0040(8)$ \\
\hline $\mathrm{C} 44$ & $0.0218(10)$ & $0.0233(13)$ & $0.0155(11)$ & $0.0008(10)$ & $0.0039(9)$ & $0.0011(10)$ \\
\hline $\mathrm{C} 20$ & $0.0105(9)$ & $0.0123(10)$ & $0.0184(11)$ & $0.0015(8)$ & $0.0010(8)$ & $-0.0046(8)$ \\
\hline $\mathrm{C} 26$ & $0.0108(8)$ & $0.0094(10)$ & $0.0193(11)$ & $-0.0032(8)$ & $0.0011(8)$ & $0.0030(8)$ \\
\hline $\mathrm{C} 35$ & $0.0109(8)$ & $0.0099(10)$ & $0.0206(11)$ & $0.0000(8)$ & $-0.0007(8)$ & $0.0034(8)$ \\
\hline $\mathrm{C} 32$ & $0.0093(8)$ & $0.0088(10)$ & $0.0185(11)$ & $-0.0033(8)$ & $-0.0011(8)$ & $-0.0006(8)$ \\
\hline $\mathrm{C} 21$ & $0.0075(8)$ & $0.0148(11)$ & $0.0198(11)$ & $-0.0011(8)$ & $0.0017(8)$ & $0.0048(9)$ \\
\hline N5 & $0.0251(10)$ & $0.0215(11)$ & $0.0201(11)$ & $0.0007(9)$ & $-0.0033(8)$ & $-0.0015(9)$ \\
\hline $\mathrm{C} 47$ & $0.0215(10)$ & $0.0227(13)$ & $0.0142(11)$ & $-0.0004(10)$ & $-0.0039(9)$ & $-0.0015(10)$ \\
\hline N8 & $0.0252(10)$ & $0.0211(11)$ & $0.0207(11)$ & $0.0001(9)$ & $0.0031(8)$ & $0.0006(9)$ \\
\hline C12 & $0.0098(9)$ & $0.0094(10)$ & $0.0178(11)$ & $-0.0034(8)$ & $-0.0002(8)$ & $-0.0019(8)$ \\
\hline C39 & $0.0203(10)$ & $0.0224(12)$ & $0.0171(12)$ & $-0.0001(9)$ & $-0.0017(9)$ & $0.0001(10)$ \\
\hline $\mathrm{C} 8$ & $0.0065(8)$ & $0.0087(10)$ & $0.0118(10)$ & $-0.0016(7)$ & $-0.0003(7)$ & $-0.0011(8)$ \\
\hline $\mathrm{C} 29$ & $0.0104(8)$ & $0.0101(10)$ & $0.0163(11)$ & $0.0009(8)$ & $0.0005(8)$ & $-0.0008(8)$ \\
\hline C19 & $0.0210(10)$ & $0.0209(12)$ & $0.0189(12)$ & $-0.0006(9)$ & $0.0003(9)$ & $-0.0007(10)$ \\
\hline $\mathrm{C} 15$ & $0.0107(8)$ & $0.0102(10)$ & $0.0216(11)$ & $0.0003(8)$ & $0.0002(8)$ & $-0.0039(9)$ \\
\hline $\mathrm{C} 28$ & $0.0051(8)$ & $0.0103(10)$ & $0.0114(10)$ & $-0.0023(7)$ & $-0.0014(7)$ & $-0.0004(8)$ \\
\hline $\mathrm{C} 41$ & $0.0408(14)$ & $0.0288(15)$ & $0.0138(12)$ & $-0.0106(12)$ & $-0.0012(11)$ & $-0.0015(11)$ \\
\hline
\end{tabular}




\begin{tabular}{|c|c|c|c|c|c|c|}
\hline C9 & $0.0096(8)$ & $0.0105(10)$ & $0.0174(11)$ & $0.0012(8)$ & $-0.0016(8)$ & $0.0002(8)$ \\
\hline $\mathrm{C} 1$ & $0.0077(8)$ & $0.0137(11)$ & $0.0193(11)$ & $-0.0017(8)$ & $-0.0018(8)$ & $-0.0044(9)$ \\
\hline $\mathrm{C} 5$ & $0.0227(11)$ & $0.0393(16)$ & $0.0147(12)$ & $0.0034(11)$ & $-0.0009(9)$ & $-0.0032(11)$ \\
\hline C34 & $0.0107(8)$ & $0.0086(10)$ & $0.0206(11)$ & $-0.0002(8)$ & $-0.0011(8)$ & $0.0003(9)$ \\
\hline $\mathrm{C} 23$ & $0.0234(11)$ & $0.0392(16)$ & $0.0148(12)$ & $0.0030(11)$ & $-0.0003(9)$ & $0.0014(11)$ \\
\hline C6 & $0.0162(10)$ & $0.0263(13)$ & $0.0185(12)$ & $0.0020(9)$ & $-0.0047(9)$ & $-0.0004(10)$ \\
\hline $\mathrm{C} 50$ & $0.0402(14)$ & $0.0292(15)$ & $0.0138(12)$ & $-0.0104(12)$ & $0.0003(11)$ & $0.0013(11)$ \\
\hline $\mathrm{C} 25$ & $0.0140(9)$ & $0.0187(12)$ & $0.0248(13)$ & $0.0034(9)$ & $0.0043(9)$ & $0.0065(10)$ \\
\hline C52 & $0.0312(13)$ & $0.0275(15)$ & $0.0292(14)$ & $0.0091(12)$ & $0.0037(11)$ & $0.0028(12)$ \\
\hline $\mathrm{C} 3$ & $0.0146(9)$ & $0.0185(12)$ & $0.0247(12)$ & $0.0035(9)$ & $-0.0047(9)$ & $-0.0071(10)$ \\
\hline C36 & $0.0204(11)$ & $0.0192(12)$ & $0.0281(13)$ & $-0.0040(9)$ & $0.0011(10)$ & $0.0060(10)$ \\
\hline C43 & $0.0304(12)$ & $0.0271(14)$ & $0.0286(14)$ & $0.0083(12)$ & $-0.0035(11)$ & -0.0032 \\
\hline $\mathrm{C} 14$ & $0.0109(8)$ & $0.0079(10)$ & $0.0208(11)$ & $0.0000(8)$ & $0.0007(8)$ & $-0.0002(8)$ \\
\hline $\mathrm{C} 7$ & $0.0102(8)$ & $0.0072(10)$ & $0.0219(11)$ & $-0.0015(8)$ & $-0.0005(8)$ & $-0.0007(8)$ \\
\hline $\mathrm{C} 27$ & $0.0105(8)$ & $0.0076(10)$ & $0.0218(11)$ & $-0.0011(8)$ & $0.0003(8)$ & $0.0005(8)$ \\
\hline $\mathrm{C} 22$ & $0.0171(10)$ & $0.0254(13)$ & $0.0178(12)$ & $0.0025(9)$ & $0.0028(9)$ & $-0.0017(10)$ \\
\hline C38 & $0.0296(12)$ & $0.0329(15)$ & $0.0179(12)$ & $0.0003(11)$ & $0.0042(10)$ & $0.0028(11)$ \\
\hline $\mathrm{C} 18$ & $0.0309(12)$ & $0.0313(15)$ & $0.0188(12)$ & $-0.0002(11)$ & $-0.0055(10)$ & $-0.0051(11)$ \\
\hline $\mathrm{C} 16$ & $0.0185(10)$ & $0.0188(12)$ & $0.0281(13)$ & $-0.0041(9)$ & $0.0002(9)$ & $-0.0065(10)$ \\
\hline $\mathrm{C} 17$ & $0.0278(12)$ & $0.0337(16)$ & $0.0249(14)$ & $-0.0030(12)$ & $-0.0078(11)$ & $-0.0119(12)$ \\
\hline C37 & $0.0288(12)$ & $0.0344(16)$ & $0.0253(14)$ & $-0.0025(12)$ & $0.0078(11)$ & $0.0125(12)$ \\
\hline $\mathrm{C} 4$ & $0.0217(11)$ & $0.0379(16)$ & $0.0242(13)$ & $0.0118(11)$ & $-0.0006(10)$ & $-0.0115(12)$ \\
\hline $\mathrm{C} 46$ & $0.0319(13)$ & $0.0318(15)$ & $0.0278(14)$ & $0.0052(12)$ & $0.0051(11)$ & $-0.0110(12)$ \\
\hline C24 & $0.0222(11)$ & $0.0377(16)$ & $0.0230(13)$ & $0.0120(11)$ & $0.0008(10)$ & $0.0114(12)$ \\
\hline $\mathrm{C} 42$ & $0.0288(13)$ & $0.0324(16)$ & $0.0326(15)$ & $0.0062(12)$ & $-0.0088(11)$ & $-0.0059(12)$ \\
\hline C51 & $0.0274(12)$ & $0.0325(16)$ & $0.0341(15)$ & $0.0052(12)$ & $0.0079(11)$ & $0.0056(12)$ \\
\hline $\mathrm{C} 11$ & $0.0069(8)$ & $0.0103(11)$ & $0.0280(13)$ & $-0.0050(8)$ & $0.0018(8)$ & $-0.0036(9)$ \\
\hline C48 & $0.0536(19)$ & $0.051(2)$ & $0.0385(19)$ & $-0.0346(17)$ & $-0.0143(16)$ & $0.0156(16)$ \\
\hline $\mathrm{C} 30$ & $0.0081(8)$ & $0.0162(11)$ & $0.0231(12)$ & $0.0023(8)$ & $0.0027(8)$ & $0.0029(9)$ \\
\hline $\mathrm{C} 31$ & $0.0074(8)$ & $0.0113(11)$ & $0.0275(13)$ & $-0.0050(8)$ & $-0.0029(8)$ & $0.0022(9)$ \\
\hline C49 & $0.0310(13)$ & $0.0319(15)$ & $0.0276(14)$ & $0.0049(12)$ & $-0.0063(11)$ & $0.0108(12)$ \\
\hline $\mathrm{C} 45$ & $0.0540(19)$ & $0.050(2)$ & $0.043(2)$ & $-0.0335(18)$ & $0.0155(16)$ & $-0.0181(16)$ \\
\hline $\mathrm{C} 10$ & $0.0081(8)$ & $0.0165(11)$ & $0.0224(12)$ & $0.0014(8)$ & $-0.0033(8)$ & $-0.0035(9)$ \\
\hline
\end{tabular}

Geometric parameters $\left(\AA,{ }^{\circ}\right)$

\begin{tabular}{|c|c|c|c|}
\hline $\mathrm{Mo} 01-\mathrm{O} 1$ & $1.9567(16)$ & $\mathrm{C} 29-\mathrm{C} 30$ & $1.311(3)$ \\
\hline $\mathrm{Mo01-O}$ & $1.6769(16)$ & $\mathrm{C} 19-\mathrm{H} 012$ & 0.9500 \\
\hline $\mathrm{Mo} 01-\mathrm{N} 2$ & $2.2493(17)$ & $\mathrm{C} 19-\mathrm{C} 18$ & $1.322(4)$ \\
\hline $\mathrm{Mo01-O4}$ & $1.7518(14)$ & $\mathrm{C} 15-\mathrm{C} 14$ & $1.512(3)$ \\
\hline $\mathrm{Mo01-O} 2$ & $1.9213(16)$ & $\mathrm{C} 15-\mathrm{C} 16$ & $1.324(3)$ \\
\hline $\mathrm{Mo01-N1}$ & $2.3475(16)$ & $\mathrm{C} 41-\mathrm{H} 015$ & 0.9500 \\
\hline $\mathrm{Mo} 02-\mathrm{O} 5$ & $1.9665(15)$ & C9- $\mathrm{H} 016$ & 0.9500 \\
\hline $\mathrm{Mo} 02-\mathrm{O} 8$ & $1.7493(15)$ & $\mathrm{C} 9-\mathrm{C} 10$ & $1.335(3)$ \\
\hline $\mathrm{Mo02}-\mathrm{O} 7$ & $1.6423(17)$ & $\mathrm{C} 1-\mathrm{C} 6$ & $1.407(3)$ \\
\hline $\mathrm{Mo} 02-\mathrm{N} 4$ & $2.2145(18)$ & $\mathrm{C} 5-\mathrm{H} 018$ & 0.9500 \\
\hline $\mathrm{Mo02-O6}$ & $1.9692(15)$ & $\mathrm{C} 5-\mathrm{C} 6$ & $1.410(3)$ \\
\hline $\mathrm{Mo} 02-\mathrm{N} 3$ & $2.3529(16)$ & $\mathrm{C} 5-\mathrm{C} 4$ & $1.426(4)$ \\
\hline
\end{tabular}




\begin{tabular}{|c|c|c|c|}
\hline $\mathrm{O} 5-\mathrm{C} 21$ & $1.368(2)$ & $\mathrm{C} 34-\mathrm{H} 01 \mathrm{G}$ & 0.9900 \\
\hline $\mathrm{O} 1-\mathrm{C} 1$ & $1.377(2)$ & $\mathrm{C} 34-\mathrm{H} 01 \mathrm{H}$ & 0.9900 \\
\hline N2-H008 & 1.0000 & $\mathrm{C} 23-\mathrm{H} 01 \mathrm{I}$ & 0.9500 \\
\hline $\mathrm{N} 2-\mathrm{C} 13$ & $1.465(2)$ & $\mathrm{C} 23-\mathrm{C} 22$ & $1.401(3)$ \\
\hline $\mathrm{N} 2-\mathrm{C} 14$ & $1.492(3)$ & $\mathrm{C} 23-\mathrm{C} 24$ & $1.420(4)$ \\
\hline N4-H009 & 1.0000 & C6-H01B & 0.9500 \\
\hline $\mathrm{N} 4-\mathrm{C} 33$ & $1.468(2)$ & $\mathrm{C} 50-\mathrm{H} 3 \mathrm{AA}$ & 0.9500 \\
\hline $\mathrm{N} 4-\mathrm{C} 34$ & $1.525(3)$ & $\mathrm{C} 25-\mathrm{H} 01 \mathrm{~J}$ & 0.9500 \\
\hline $\mathrm{O} 2-\mathrm{C} 20$ & $1.295(3)$ & $\mathrm{C} 25-\mathrm{C} 24$ & $1.370(4)$ \\
\hline $\mathrm{O} 6-\mathrm{C} 40$ & $1.332(3)$ & $\mathrm{C} 52-\mathrm{H} 4 \mathrm{AA}$ & 0.9800 \\
\hline $\mathrm{N} 1-\mathrm{C} 8$ & $1.389(3)$ & $\mathrm{C} 52-\mathrm{H}$ & 0.9800 \\
\hline $\mathrm{N} 1-\mathrm{C} 7$ & $1.486(3)$ & C $52-\mathrm{HA}$ & 0.9800 \\
\hline $\mathrm{N} 1-\mathrm{H} 00 \mathrm{D}$ & $0.85(3)$ & $\mathrm{C} 3-\mathrm{H} 01 \mathrm{~F}$ & 0.9500 \\
\hline $\mathrm{N} 3-\mathrm{C} 28$ & $1.379(3)$ & $\mathrm{C} 3-\mathrm{C} 4$ & $1.399(4)$ \\
\hline $\mathrm{N} 3-\mathrm{C} 27$ & $1.519(3)$ & $\mathrm{C} 36-\mathrm{H} 01 \mathrm{~K}$ & 0.9500 \\
\hline N3-H00E & $0.79(3)$ & C36-C37 & $1.393(4)$ \\
\hline $\mathrm{O} 9-\mathrm{C} 41$ & $1.250(4)$ & $\mathrm{C} 43-\mathrm{H} 01 \mathrm{~V}$ & 0.9800 \\
\hline $\mathrm{O} 10-\mathrm{C} 44$ & $1.169(3)$ & $\mathrm{C} 43-\mathrm{H} 01 \mathrm{X}$ & 0.9800 \\
\hline $\mathrm{O} 11-\mathrm{C} 47$ & $1.187(3)$ & C43-H01 & 0.9800 \\
\hline $\mathrm{O} 12-\mathrm{C} 50$ & $1.249(4)$ & $\mathrm{C} 14-\mathrm{H} 01 \mathrm{~A}$ & 0.9900 \\
\hline $\mathrm{C} 40-\mathrm{C} 35$ & $1.338(3)$ & $\mathrm{C} 14-\mathrm{H} 01 \mathrm{C}$ & 0.9900 \\
\hline $\mathrm{C} 40-\mathrm{C} 39$ & $1.405(3)$ & C7-H01D & 0.9900 \\
\hline $\mathrm{C} 33-\mathrm{C} 32$ & $1.307(3)$ & C7-H01E & 0.9900 \\
\hline $\mathrm{C} 33-\mathrm{C} 28$ & $1.392(3)$ & C27-H01L & 0.9900 \\
\hline $\mathrm{N} 6-\mathrm{C} 44$ & $1.332(3)$ & $\mathrm{C} 27-\mathrm{H} 01 \mathrm{M}$ & 0.9900 \\
\hline N6-C46 & $1.464(3)$ & $\mathrm{C} 22-\mathrm{H} 01 \mathrm{Q}$ & 0.9500 \\
\hline N6-C45 & $1.377(4)$ & $\mathrm{C} 38-\mathrm{H} 01 \mathrm{~S}$ & 0.9500 \\
\hline $\mathrm{C} 13-\mathrm{C} 12$ & $1.333(3)$ & C38-C37 & $1.333(4)$ \\
\hline $\mathrm{C} 13-\mathrm{C} 8$ & $1.419(3)$ & $\mathrm{C} 18-\mathrm{H} 01 \mathrm{~N}$ & 0.9500 \\
\hline $\mathrm{N} 7-\mathrm{C} 47$ & $1.355(3)$ & $\mathrm{C} 18-\mathrm{C} 17$ & $1.372(4)$ \\
\hline $\mathrm{N} 7-\mathrm{C} 48$ & $1.394(4)$ & $\mathrm{C} 16-\mathrm{H} 01 \mathrm{O}$ & 0.9500 \\
\hline N7-C49 & $1.443(3)$ & $\mathrm{C} 16-\mathrm{C} 17$ & $1.396(4)$ \\
\hline $\mathrm{C} 2-\mathrm{C} 1$ & $1.430(3)$ & C17-H01P & 0.9500 \\
\hline $\mathrm{C} 2-\mathrm{C} 3$ & $1.415(3)$ & C37-H01T & 0.9500 \\
\hline $\mathrm{C} 2-\mathrm{C} 7$ & $1.515(3)$ & $\mathrm{C} 4-\mathrm{H} 01 \mathrm{R}$ & 0.9500 \\
\hline $\mathrm{C} 44-\mathrm{H} 00 \mathrm{P}$ & 0.9500 & $\mathrm{C} 46-\mathrm{H} 1 \mathrm{AA}$ & 0.9800 \\
\hline $\mathrm{C} 20-\mathrm{C} 19$ & 1.407 (3) & $\mathrm{C} 46-\mathrm{HB}$ & 0.9800 \\
\hline $\mathrm{C} 20-\mathrm{C} 15$ & $1.380(3)$ & $\mathrm{C} 46-\mathrm{HC}$ & 0.9800 \\
\hline $\mathrm{C} 26-\mathrm{C} 21$ & $1.425(3)$ & $\mathrm{C} 24-\mathrm{H} 01 \mathrm{U}$ & 0.9500 \\
\hline $\mathrm{C} 26-\mathrm{C} 25$ & $1.410(3)$ & $\mathrm{C} 42-\mathrm{H} 0 \mathrm{AA}$ & 0.9800 \\
\hline $\mathrm{C} 26-\mathrm{C} 27$ & $1.490(3)$ & $\mathrm{C} 42-\mathrm{HD}$ & 0.9800 \\
\hline $\mathrm{C} 35-\mathrm{C} 34$ & $1.513(3)$ & $\mathrm{C} 42-\mathrm{HE}$ & 0.9800 \\
\hline $\mathrm{C} 35-\mathrm{C} 36$ & $1.373(3)$ & C $51-\mathrm{H} 5 \mathrm{AA}$ & 0.9800 \\
\hline $\mathrm{C} 32-\mathrm{H} 00 \mathrm{~T}$ & 0.9500 & $\mathrm{C} 51-\mathrm{HF}$ & 0.9800 \\
\hline $\mathrm{C} 32-\mathrm{C} 31$ & $1.412(3)$ & $\mathrm{C} 51-\mathrm{HG}$ & 0.9800 \\
\hline $\mathrm{C} 21-\mathrm{C} 22$ & $1.388(3)$ & $\mathrm{C} 11-\mathrm{H} 01 \mathrm{~W}$ & 0.9500 \\
\hline N5-C41 & $1.280(4)$ & $\mathrm{C} 11-\mathrm{C} 10$ & $1.424(3)$ \\
\hline N5-C43 & $1.482(3)$ & $\mathrm{C} 48-\mathrm{H} 2 \mathrm{AA}$ & 0.9800 \\
\hline
\end{tabular}




\begin{tabular}{|c|c|c|c|}
\hline $\mathrm{N} 5-\mathrm{C} 42$ & $1.423(3)$ & $\mathrm{C} 48-\mathrm{HH}$ & 0.9800 \\
\hline C47-H00W & 0.9500 & $\mathrm{C} 48-\mathrm{HI}$ & 0.9800 \\
\hline $\mathrm{N} 8-\mathrm{C} 50$ & $1.308(3)$ & $\mathrm{C} 30-\mathrm{H} 01 \mathrm{Y}$ & 0.9500 \\
\hline $\mathrm{N} 8-\mathrm{C} 52$ & $1.481(3)$ & $\mathrm{C} 30-\mathrm{C} 31$ & $1.391(3)$ \\
\hline $\mathrm{N} 8-\mathrm{C} 51$ & $1.442(3)$ & $\mathrm{C} 31-\mathrm{H} 01 \mathrm{Z}$ & 0.9500 \\
\hline $\mathrm{C} 12-\mathrm{H} 00 \mathrm{Y}$ & 0.9500 & $\mathrm{C} 49-\mathrm{H} 02 \mathrm{D}$ & 0.9800 \\
\hline $\mathrm{C} 12-\mathrm{C} 11$ & $1.405(3)$ & $\mathrm{C} 49-\mathrm{H} 02 \mathrm{E}$ & 0.9800 \\
\hline $\mathrm{C} 39-\mathrm{H} 00 \mathrm{Z}$ & 0.9500 & $\mathrm{C} 49-\mathrm{H} 02 \mathrm{~F}$ & 0.9800 \\
\hline C39-C38 & $1.364(4)$ & $\mathrm{C} 45-\mathrm{H} 02 \mathrm{~A}$ & 0.9800 \\
\hline $\mathrm{C} 8-\mathrm{C} 9$ & $1.403(2)$ & $\mathrm{C} 45-\mathrm{H} 02 \mathrm{~B}$ & 0.9800 \\
\hline С29-H011 & 0.9500 & $\mathrm{C} 45-\mathrm{H} 02 \mathrm{C}$ & 0.9800 \\
\hline $\mathrm{C} 29-\mathrm{C} 28$ & $1.410(2)$ & $\mathrm{C} 10-\mathrm{H} 022$ & 0.9500 \\
\hline $\mathrm{O} 1-\mathrm{Mo} 01-\mathrm{N} 2$ & $77.65(7)$ & $\mathrm{O} 1-\mathrm{C} 1-\mathrm{C} 6$ & $118.21(19)$ \\
\hline $\mathrm{O} 1-\mathrm{Mo} 01-\mathrm{N} 1$ & $80.23(6)$ & $\mathrm{C} 6-\mathrm{C} 1-\mathrm{C} 2$ & $120.4(2)$ \\
\hline $\mathrm{O} 3-\mathrm{Mo} 01-\mathrm{O} 1$ & $100.51(8)$ & $\mathrm{C} 6-\mathrm{C} 5-\mathrm{H} 018$ & 119.6 \\
\hline $\mathrm{O} 3-\mathrm{Mo} 01-\mathrm{N} 2$ & $161.90(6)$ & $\mathrm{C} 6-\mathrm{C} 5-\mathrm{C} 4$ & $120.9(2)$ \\
\hline $\mathrm{O} 3-\mathrm{Mo} 01-\mathrm{O} 4$ & $109.80(7)$ & $\mathrm{C} 4-\mathrm{C} 5-\mathrm{H} 018$ & 119.6 \\
\hline $\mathrm{O} 3-\mathrm{Mo} 01-\mathrm{O} 2$ & $92.51(8)$ & $\mathrm{N} 4-\mathrm{C} 34-\mathrm{H} 01 \mathrm{G}$ & 109.6 \\
\hline $\mathrm{O} 3-\mathrm{Mo} 01-\mathrm{N} 1$ & $86.75(6)$ & $\mathrm{N} 4-\mathrm{C} 34-\mathrm{H} 01 \mathrm{H}$ & 109.6 \\
\hline $\mathrm{N} 2-\mathrm{Mo} 01-\mathrm{N} 1$ & $75.18(6)$ & $\mathrm{C} 35-\mathrm{C} 34-\mathrm{N} 4$ & $110.35(17)$ \\
\hline $\mathrm{O} 4-\mathrm{Mo} 01-\mathrm{O} 1$ & $94.14(7)$ & $\mathrm{C} 35-\mathrm{C} 34-\mathrm{H} 01 \mathrm{G}$ & 109.6 \\
\hline $\mathrm{O} 4-\mathrm{Mo} 01-\mathrm{N} 2$ & $88.30(6)$ & $\mathrm{C} 35-\mathrm{C} 34-\mathrm{H} 01 \mathrm{H}$ & 109.6 \\
\hline $\mathrm{O} 4-\mathrm{Mo} 01-\mathrm{O} 2$ & $98.32(7)$ & $\mathrm{H} 01 \mathrm{G}-\mathrm{C} 34-\mathrm{H} 01 \mathrm{H}$ & 108.1 \\
\hline $\mathrm{O} 4-\mathrm{Mo} 01-\mathrm{N} 1$ & $163.31(7)$ & $\mathrm{C} 22-\mathrm{C} 23-\mathrm{H} 01 \mathrm{I}$ & 119.1 \\
\hline $\mathrm{O} 2-\mathrm{Mo} 01-\mathrm{O} 1$ & $157.78(6)$ & $\mathrm{C} 22-\mathrm{C} 23-\mathrm{C} 24$ & $121.9(2)$ \\
\hline $\mathrm{O} 2-\mathrm{Mo} 01-\mathrm{N} 2$ & $84.38(7)$ & $\mathrm{C} 24-\mathrm{C} 23-\mathrm{H} 01 \mathrm{I}$ & 119.1 \\
\hline $\mathrm{O} 2-\mathrm{Mo} 01-\mathrm{N} 1$ & $82.63(6)$ & $\mathrm{C} 1-\mathrm{C} 6-\mathrm{C} 5$ & $119.3(2)$ \\
\hline $\mathrm{O} 5-\mathrm{Mo} 02-\mathrm{N} 4$ & $81.39(7)$ & $\mathrm{C} 1-\mathrm{C} 6-\mathrm{H} 01 \mathrm{~B}$ & 120.4 \\
\hline $\mathrm{O} 5-\mathrm{Mo} 02-\mathrm{O} 6$ & $158.10(6)$ & $\mathrm{C} 5-\mathrm{C} 6-\mathrm{H} 01 \mathrm{~B}$ & 120.4 \\
\hline $\mathrm{O} 5-\mathrm{Mo} 02-\mathrm{N} 3$ & $81.07(6)$ & $\mathrm{O} 12-\mathrm{C} 50-\mathrm{N} 8$ & $125.0(3)$ \\
\hline $\mathrm{O} 8-\mathrm{Mo} 02-\mathrm{O} 5$ & $94.30(7)$ & $\mathrm{O} 12-\mathrm{C} 50-\mathrm{H} 3 \mathrm{AA}$ & 117.5 \\
\hline $\mathrm{O} 8-\mathrm{Mo} 02-\mathrm{N} 4$ & $89.52(7)$ & $\mathrm{N} 8-\mathrm{C} 50-\mathrm{H} 3 \mathrm{AA}$ & 117.5 \\
\hline $\mathrm{O} 8-\mathrm{Mo} 02-\mathrm{O} 6$ & $98.33(7)$ & $\mathrm{C} 26-\mathrm{C} 25-\mathrm{H} 01 \mathrm{~J}$ & 120.0 \\
\hline $\mathrm{O} 8-\mathrm{Mo} 02-\mathrm{N} 3$ & $163.76(7)$ & $\mathrm{C} 24-\mathrm{C} 25-\mathrm{C} 26$ & $120.0(2)$ \\
\hline $\mathrm{O} 7-\mathrm{Mo} 02-\mathrm{O} 5$ & $96.96(8)$ & $\mathrm{C} 24-\mathrm{C} 25-\mathrm{H} 01 \mathrm{~J}$ & 120.0 \\
\hline $\mathrm{O} 7-\mathrm{Mo} 02-\mathrm{O} 8$ & $109.00(7)$ & $\mathrm{N} 8-\mathrm{C} 52-\mathrm{H} 4 \mathrm{AA}$ & 109.5 \\
\hline $\mathrm{O} 7-\mathrm{Mo} 02-\mathrm{N} 4$ & $161.48(7)$ & $\mathrm{N} 8-\mathrm{C} 52-\mathrm{H}$ & 109.5 \\
\hline $\mathrm{O} 7-\mathrm{Mo} 02-\mathrm{O} 6$ & $95.73(8)$ & $\mathrm{N} 8-\mathrm{C} 52-\mathrm{HA}$ & 109.5 \\
\hline $\mathrm{O} 7-\mathrm{Mo} 02-\mathrm{N} 3$ & $87.08(7)$ & $\mathrm{H} 4 \mathrm{AA}-\mathrm{C} 52-\mathrm{H}$ & 109.5 \\
\hline $\mathrm{N} 4-\mathrm{Mo} 02-\mathrm{N} 3$ & $74.43(6)$ & $\mathrm{H} 4 \mathrm{AA}-\mathrm{C} 52-\mathrm{HA}$ & 109.5 \\
\hline $\mathrm{O} 6-\mathrm{Mo} 02-\mathrm{N} 4$ & $80.91(7)$ & $\mathrm{H}-\mathrm{C} 52-\mathrm{HA}$ & 109.5 \\
\hline $\mathrm{O} 6-\mathrm{Mo} 02-\mathrm{N} 3$ & $81.84(6)$ & $\mathrm{C} 2-\mathrm{C} 3-\mathrm{H} 01 \mathrm{~F}$ & 119.8 \\
\hline $\mathrm{C} 21-\mathrm{O} 5-\mathrm{Mo} 02$ & $137.80(14)$ & $\mathrm{C} 4-\mathrm{C} 3-\mathrm{C} 2$ & $120.5(2)$ \\
\hline $\mathrm{C} 1-\mathrm{O} 1-\mathrm{Mo0} 1$ & $138.48(14)$ & $\mathrm{C} 4-\mathrm{C} 3-\mathrm{H} 01 \mathrm{~F}$ & 119.8 \\
\hline $\mathrm{Mo} 01-\mathrm{N} 2-\mathrm{H} 008$ & 107.3 & $\mathrm{C} 35-\mathrm{C} 36-\mathrm{H} 01 \mathrm{~K}$ & 118.3 \\
\hline $\mathrm{C} 13-\mathrm{N} 2-\mathrm{Mo} 01$ & $112.59(12)$ & $\mathrm{C} 35-\mathrm{C} 36-\mathrm{C} 37$ & $123.5(3)$ \\
\hline $\mathrm{C} 13-\mathrm{N} 2-\mathrm{H} 008$ & 107.3 & $\mathrm{C} 37-\mathrm{C} 36-\mathrm{H} 01 \mathrm{~K}$ & 118.3 \\
\hline
\end{tabular}




\begin{tabular}{|c|c|}
\hline $\mathrm{C} 13-\mathrm{N} 2-\mathrm{C} 14$ & $113.36(16)$ \\
\hline $\mathrm{C} 14-\mathrm{N} 2-\mathrm{Mo} 01$ & $108.75(12)$ \\
\hline $\mathrm{C} 14-\mathrm{N} 2-\mathrm{H} 008$ & 107.3 \\
\hline Mo02-N4-H009 & 107.0 \\
\hline $\mathrm{C} 33-\mathrm{N} 4-\mathrm{Mo02}$ & $113.03(13)$ \\
\hline C33-N4-H009 & 107.0 \\
\hline C33-N4-C34 & $111.85(15)$ \\
\hline $\mathrm{C} 34-\mathrm{N} 4-\mathrm{Mo02}$ & $110.51(12)$ \\
\hline C34-N4-H009 & 107.0 \\
\hline $\mathrm{C} 20-\mathrm{O} 2-\mathrm{Mo} 01$ & $136.10(14)$ \\
\hline $\mathrm{C} 40-\mathrm{O} 6-\mathrm{Mo02}$ & $138.81(13)$ \\
\hline $\mathrm{Mo01-N1-H00D}$ & $110.7(19)$ \\
\hline $\mathrm{C} 8-\mathrm{N} 1-\mathrm{Mo0} 1$ & $113.88(13)$ \\
\hline $\mathrm{C} 8-\mathrm{N} 1-\mathrm{C} 7$ & $107.69(16)$ \\
\hline $\mathrm{C} 8-\mathrm{N} 1-\mathrm{H} 00 \mathrm{D}$ & $106(2)$ \\
\hline $\mathrm{C} 7-\mathrm{N} 1-\mathrm{Mo0} 1$ & $112.87(12)$ \\
\hline $\mathrm{C} 7-\mathrm{N} 1-\mathrm{H} 00 \mathrm{D}$ & $105(2)$ \\
\hline $\mathrm{Mo} 02-\mathrm{N} 3-\mathrm{H} 00 \mathrm{E}$ & $113.0(19)$ \\
\hline $\mathrm{C} 28-\mathrm{N} 3-\mathrm{Mo0} 2$ & $114.65(13)$ \\
\hline $\mathrm{C} 28-\mathrm{N} 3-\mathrm{C} 27$ & $109.21(16)$ \\
\hline $\mathrm{C} 28-\mathrm{N} 3-\mathrm{H} 00 \mathrm{E}$ & $99(2)$ \\
\hline $\mathrm{C} 27-\mathrm{N} 3-\mathrm{Mo} 02$ & $111.45(12)$ \\
\hline $\mathrm{C} 27-\mathrm{N} 3-\mathrm{H} 00 \mathrm{E}$ & $109(2)$ \\
\hline $\mathrm{O} 6-\mathrm{C} 40-\mathrm{C} 35$ & $118.3(2)$ \\
\hline $\mathrm{O} 6-\mathrm{C} 40-\mathrm{C} 39$ & $121.4(2)$ \\
\hline $\mathrm{C} 35-\mathrm{C} 40-\mathrm{C} 39$ & $120.3(2)$ \\
\hline $\mathrm{C} 32-\mathrm{C} 33-\mathrm{N} 4$ & $121.34(19)$ \\
\hline $\mathrm{C} 32-\mathrm{C} 33-\mathrm{C} 28$ & $117.14(18)$ \\
\hline $\mathrm{C} 28-\mathrm{C} 33-\mathrm{N} 4$ & $121.41(18)$ \\
\hline $\mathrm{C} 44-\mathrm{N} 6-\mathrm{C} 46$ & $124.5(2)$ \\
\hline $\mathrm{C} 44-\mathrm{N} 6-\mathrm{C} 45$ & $120.4(2)$ \\
\hline $\mathrm{C} 45-\mathrm{N} 6-\mathrm{C} 46$ & $115.0(3)$ \\
\hline $\mathrm{C} 12-\mathrm{C} 13-\mathrm{N} 2$ & $120.75(18)$ \\
\hline $\mathrm{C} 12-\mathrm{C} 13-\mathrm{C} 8$ & $118.66(17)$ \\
\hline $\mathrm{C} 8-\mathrm{C} 13-\mathrm{N} 2$ & $120.47(17)$ \\
\hline $\mathrm{C} 47-\mathrm{N} 7-\mathrm{C} 48$ & $121.9(2)$ \\
\hline C47-N7-C49 & $124.1(2)$ \\
\hline $\mathrm{C} 48-\mathrm{N} 7-\mathrm{C} 49$ & $113.9(2)$ \\
\hline $\mathrm{C} 1-\mathrm{C} 2-\mathrm{C} 7$ & $119.57(18)$ \\
\hline $\mathrm{C} 3-\mathrm{C} 2-\mathrm{C} 1$ & $119.5(2)$ \\
\hline $\mathrm{C} 3-\mathrm{C} 2-\mathrm{C} 7$ & $120.89(19)$ \\
\hline $\mathrm{O} 10-\mathrm{C} 44-\mathrm{N} 6$ & $125.1(2)$ \\
\hline $\mathrm{O} 10-\mathrm{C} 44-\mathrm{H} 00 \mathrm{P}$ & 117.5 \\
\hline $\mathrm{N} 6-\mathrm{C} 44-\mathrm{H} 00 \mathrm{P}$ & 117.5 \\
\hline $\mathrm{O} 2-\mathrm{C} 20-\mathrm{C} 19$ & $118.2(2)$ \\
\hline $\mathrm{O} 2-\mathrm{C} 20-\mathrm{C} 15$ & $118.5(2)$ \\
\hline $\mathrm{C} 15-\mathrm{C} 20-\mathrm{C} 19$ & $123.3(2)$ \\
\hline $\mathrm{C} 21-\mathrm{C} 26-\mathrm{C} 27$ & $119.07(18)$ \\
\hline
\end{tabular}

$\begin{array}{ll}\text { N5-C } 43-\mathrm{H} 01 \mathrm{~V} & 109.5 \\ \text { N5-C43-H01X } & 109.5 \\ \text { N5-C43-H01 } & 109.5 \\ \text { H01V-C43-H01X } & 109.5 \\ \text { H01V-C43-H01 } & 109.5 \\ \text { H01X-C43-H01 } & 109.5 \\ \text { N2-C14-C15 } & 108.70(16) \\ \text { N2-C14-H01A } & 109.9 \\ \text { N2-C14-H01C } & 109.9 \\ \text { C15-C14-H01A } & 109.9 \\ \text { C15-C14-H01C } & 109.9 \\ \text { H01A-C14-H01C } & 108.3 \\ \text { N1-C7-C2 } & 110.47(17) \\ \text { N1-C7-H01D } & 109.6 \\ \text { N1-C7-H01E } & 109.6 \\ \text { C2-C7-H01D } & 109.6 \\ \text { C2-C7-H01E } & 109.6 \\ \text { H01D-C7-H01E } & 108.1 \\ \text { N3-C27-H01L } & 109.1 \\ \text { N3-C27-H01M } & 109.1 \\ \text { C26-C27-N3 } & 112.45(18) \\ \text { C26-C27-H01L } & 109.1 \\ \text { C26-C27-H01M } & 109.1 \\ \text { H01L-C27-H01M } & 107.8 \\ \text { C21-C22-C23 } & 118.6(2) \\ \text { C21-C22-H01Q } & 120.7 \\ \text { C23-C22-H01Q } & 120.7 \\ \text { C39-C38-H01S } & 121.2 \\ \text { C37-C38-C39 } & 117.7(2) \\ \text { C37-C38-H01S } & 121.2 \\ \text { C19-C18-H01N } & 121.0 \\ \text { C19-C18-C17 } & 118.0(2) \\ \text { C17-C18-H01N } & 121.0 \\ \text { C15-C16-H01O } & 119.6 \\ \text { C15-C16-C17 } & 1209.5 \\ \text { C17-C16-H01O } & 120.7(3) \\ \text { C18-C17-C16 } & 119.6 \\ \text { C18-C17-H01P } & 122.5(3) \\ \text { C16-C17-H01P } & 118.8 \\ \text { C36-C37-H01T } & 118.8 \\ \text { C38-C37-C36 } & 120.1 \\ \text { C38-C37-H01T } & 119.7(2) \\ \text { C5-C4-H01R } & 120.1 \\ \text { C3-C4-C5 } & 120.3 \\ \text { C3-C4-H01R } & \text { N6-C46-H1AA } \\ \text { N6-C46-HB } & \\ \text { N6-C46-HC } & \\ & \end{array}$




\begin{tabular}{|c|c|}
\hline $\mathrm{C} 25-\mathrm{C} 26-\mathrm{C} 21$ & $120.4(2)$ \\
\hline $\mathrm{C} 25-\mathrm{C} 26-\mathrm{C} 27$ & $120.5(2)$ \\
\hline $\mathrm{C} 40-\mathrm{C} 35-\mathrm{C} 34$ & $119.71(19)$ \\
\hline $\mathrm{C} 40-\mathrm{C} 35-\mathrm{C} 36$ & $116.4(2)$ \\
\hline $\mathrm{C} 36-\mathrm{C} 35-\mathrm{C} 34$ & $123.8(2)$ \\
\hline $\mathrm{C} 33-\mathrm{C} 32-\mathrm{H} 00 \mathrm{~T}$ & 120.0 \\
\hline $\mathrm{C} 33-\mathrm{C} 32-\mathrm{C} 31$ & $120.0(2)$ \\
\hline $\mathrm{C} 31-\mathrm{C} 32-\mathrm{H} 00 \mathrm{~T}$ & 120.0 \\
\hline $\mathrm{O} 5-\mathrm{C} 21-\mathrm{C} 26$ & $122.5(2)$ \\
\hline $\mathrm{O} 5-\mathrm{C} 21-\mathrm{C} 22$ & $117.6(2)$ \\
\hline $\mathrm{C} 22-\mathrm{C} 21-\mathrm{C} 26$ & $119.91(19)$ \\
\hline $\mathrm{C} 41-\mathrm{N} 5-\mathrm{C} 43$ & $123.2(2)$ \\
\hline $\mathrm{C} 41-\mathrm{N} 5-\mathrm{C} 42$ & $117.1(2)$ \\
\hline $\mathrm{C} 42-\mathrm{N} 5-\mathrm{C} 43$ & $119.7(2)$ \\
\hline $\mathrm{O} 11-\mathrm{C} 47-\mathrm{N} 7$ & $126.7(2)$ \\
\hline $\mathrm{O} 11-\mathrm{C} 47-\mathrm{H} 00 \mathrm{~W}$ & 116.6 \\
\hline $\mathrm{N} 7-\mathrm{C} 47-\mathrm{H} 00 \mathrm{~W}$ & 116.6 \\
\hline $\mathrm{C} 50-\mathrm{N} 8-\mathrm{C} 52$ & $122.4(2)$ \\
\hline $\mathrm{C} 50-\mathrm{N} 8-\mathrm{C} 51$ & $118.5(2)$ \\
\hline $\mathrm{C} 51-\mathrm{N} 8-\mathrm{C} 52$ & $119.1(2)$ \\
\hline $\mathrm{C} 13-\mathrm{C} 12-\mathrm{H} 00 \mathrm{Y}$ & 120.4 \\
\hline $\mathrm{C} 13-\mathrm{C} 12-\mathrm{C} 11$ & $119.15(19)$ \\
\hline $\mathrm{C} 11-\mathrm{C} 12-\mathrm{H} 00 \mathrm{Y}$ & 120.4 \\
\hline $\mathrm{C} 40-\mathrm{C} 39-\mathrm{H} 00 \mathrm{Z}$ & 118.8 \\
\hline $\mathrm{C} 38-\mathrm{C} 39-\mathrm{C} 40$ & $122.4(3)$ \\
\hline $\mathrm{C} 38-\mathrm{C} 39-\mathrm{H} 00 \mathrm{Z}$ & 118.8 \\
\hline $\mathrm{N} 1-\mathrm{C} 8-\mathrm{C} 13$ & $117.13(16)$ \\
\hline $\mathrm{N} 1-\mathrm{C} 8-\mathrm{C} 9$ & $120.23(18)$ \\
\hline $\mathrm{C} 9-\mathrm{C} 8-\mathrm{C} 13$ & $122.55(18)$ \\
\hline $\mathrm{C} 28-\mathrm{C} 29-\mathrm{H} 011$ & 120.3 \\
\hline $\mathrm{C} 30-\mathrm{C} 29-\mathrm{H} 011$ & 120.3 \\
\hline $\mathrm{C} 30-\mathrm{C} 29-\mathrm{C} 28$ & $119.4(2)$ \\
\hline $\mathrm{C} 20-\mathrm{C} 19-\mathrm{H} 012$ & 120.4 \\
\hline $\mathrm{C} 18-\mathrm{C} 19-\mathrm{C} 20$ & $119.1(2)$ \\
\hline $\mathrm{C} 18-\mathrm{C} 19-\mathrm{H} 012$ & 120.4 \\
\hline $\mathrm{C} 20-\mathrm{C} 15-\mathrm{C} 14$ & $122.4(2)$ \\
\hline $\mathrm{C} 16-\mathrm{C} 15-\mathrm{C} 20$ & $116.4(2)$ \\
\hline $\mathrm{C} 16-\mathrm{C} 15-\mathrm{C} 14$ & $121.2(2)$ \\
\hline $\mathrm{N} 3-\mathrm{C} 28-\mathrm{C} 33$ & $115.73(17)$ \\
\hline $\mathrm{N} 3-\mathrm{C} 28-\mathrm{C} 29$ & $121.04(19)$ \\
\hline $\mathrm{C} 33-\mathrm{C} 28-\mathrm{C} 29$ & $123.17(19)$ \\
\hline $\mathrm{O} 9-\mathrm{C} 41-\mathrm{N} 5$ & $126.1(3)$ \\
\hline $\mathrm{O} 9-\mathrm{C} 41-\mathrm{H} 015$ & 117.0 \\
\hline $\mathrm{N} 5-\mathrm{C} 41-\mathrm{H} 015$ & 117.0 \\
\hline $\mathrm{C} 8-\mathrm{C} 9-\mathrm{H} 016$ & 120.7 \\
\hline $\mathrm{C} 10-\mathrm{C} 9-\mathrm{C} 8$ & $118.57(19)$ \\
\hline $\mathrm{C} 10-\mathrm{C} 9-\mathrm{H} 016$ & 120.7 \\
\hline $\mathrm{O} 1-\mathrm{C} 1-\mathrm{C} 2$ & $121.4(2)$ \\
\hline
\end{tabular}

$\begin{array}{ll}\text { H1AA-C46-HB } & 109.5 \\ \text { H1AA-C46-HC } & 109.5 \\ \text { HB-C46-HC } & 109.5 \\ \text { C23-C24-H01U } & 120.4 \\ \text { C25-C24-C23 } & 119.3(2) \\ \text { C25-C24-H01U } & 120.4 \\ \text { N5-C42-H0AA } & 109.5 \\ \text { N5-C42-HD } & 109.5 \\ \text { N5-C42-HE } & 109.5 \\ \text { H0AA-C42-HD } & 109.5 \\ \text { H0AA-C42-HE } & 109.5 \\ \text { HD-C42-HE } & 109.5 \\ \text { N8-C51-H5AA } & 109.5 \\ \text { N8-C51-HF } & 109.5 \\ \text { N8-C51-HG } & 109.5 \\ \text { H5AA-C51-HF } & 109.5 \\ \text { H5AA-C51-HG } & 109.5 \\ \text { HF-C51-HG } & 109.5 \\ \text { C12-C11-H01W } & 119.1 \\ \text { C12-C11-C10 } & 121.85(19) \\ \text { C10-C11-H01W } & 119.1 \\ \text { N7-C48-H2AA } & 109.5 \\ \text { N7-C48-HH } & 109.5 \\ \text { N7-C48-HI } & 109.5 \\ \text { H2AA-C48-HH } & 109.5 \\ \text { H2AA-C48-HI } & 109.5 \\ \text { HH-C48-HI } & 109.5 \\ \text { C29-C30-H01Y } & 121.2 \\ \text { C29-C30-C31 } & 117.66(19) \\ \text { C31-C30-H01Y } & 121.2 \\ \text { C32-C31-H01Z } & 118.7 \\ \text { C30-C31-C32 } & 122.6(2) \\ \text { C30-C31-H01Z } & 118.7 \\ \text { N7-C49-H02D } & 109.5 \\ \text { N7-C49-H02E } & 109.5 \\ \text { N7-C49-H02F } & 109.5 \\ \text { H02D-C49-H02E } & 109.5 \\ \text { H02D-C49-H02F } & 109.5 \\ \text { H02E-C49-H02F } & 109.5 \\ \text { N6-C45-H02A } & 109.5 \\ \text { N6-C45-H02B } & 109.5 \\ \text { N6-C45-H02C } & 109.5 \\ \text { H02A-C45-H02B } & 109.5 \\ \text { H02A-C45-H02C } & 109.5 \\ \text { H02B-C45-H02C } & 109.5 \\ \text { C9-C10-C11 } & 19.12(18) \\ \text { C9-C10-H022 } & \\ \text { C11-C10-H022 } & \\ & \end{array}$




\begin{tabular}{|c|c|c|c|}
\hline $\mathrm{Mo} 01-\mathrm{O} 1-\mathrm{C} 1-\mathrm{C} 2$ & $-28.3(3)$ & $\mathrm{C} 12-\mathrm{C} 13-\mathrm{C} 8-\mathrm{C} 9$ & $-3.3(3)$ \\
\hline $\mathrm{Mo} 01-\mathrm{O} 1-\mathrm{C} 1-\mathrm{C} 6$ & $152.37(19)$ & $\mathrm{C} 12-\mathrm{C} 11-\mathrm{C} 10-\mathrm{C} 9$ & $-2.1(4)$ \\
\hline $\mathrm{Mo} 01-\mathrm{N} 2-\mathrm{C} 13-\mathrm{C} 12$ & $-175.03(17)$ & $\mathrm{C} 39-\mathrm{C} 40-\mathrm{C} 35-\mathrm{C} 34$ & $178.42(19)$ \\
\hline $\mathrm{Mo} 01-\mathrm{N} 2-\mathrm{C} 13-\mathrm{C} 8$ & $8.9(2)$ & $\mathrm{C} 39-\mathrm{C} 40-\mathrm{C} 35-\mathrm{C} 36$ & $0.9(3)$ \\
\hline $\mathrm{Mo} 01-\mathrm{N} 2-\mathrm{C} 14-\mathrm{C} 15$ & $-69.85(16)$ & $\mathrm{C} 39-\mathrm{C} 38-\mathrm{C} 37-\mathrm{C} 36$ & $0.5(4)$ \\
\hline $\mathrm{Mo} 01-\mathrm{O} 2-\mathrm{C} 20-\mathrm{C} 19$ & $144.35(18)$ & $\mathrm{C} 8-\mathrm{N} 1-\mathrm{C} 7-\mathrm{C} 2$ & $55.7(2)$ \\
\hline $\mathrm{Mo} 01-\mathrm{O} 2-\mathrm{C} 20-\mathrm{C} 15$ & $-36.3(3)$ & $\mathrm{C} 8-\mathrm{C} 13-\mathrm{C} 12-\mathrm{C} 11$ & $2.2(3)$ \\
\hline $\mathrm{Mo} 01-\mathrm{N} 1-\mathrm{C} 8-\mathrm{C} 13$ & $6.3(2)$ & $\mathrm{C} 8-\mathrm{C} 9-\mathrm{C} 10-\mathrm{C} 11$ & $1.1(3)$ \\
\hline $\mathrm{Mo} 01-\mathrm{N} 1-\mathrm{C} 8-\mathrm{C} 9$ & $-176.91(16)$ & $\mathrm{C} 29-\mathrm{C} 30-\mathrm{C} 31-\mathrm{C} 32$ & $2.2(3)$ \\
\hline $\mathrm{Mo} 01-\mathrm{N} 1-\mathrm{C} 7-\mathrm{C} 2$ & $-70.89(17)$ & $\mathrm{C} 19-\mathrm{C} 20-\mathrm{C} 15-\mathrm{C} 14$ & $-178.5(2)$ \\
\hline $\mathrm{Mo} 02-\mathrm{O} 5-\mathrm{C} 21-\mathrm{C} 26$ & $28.4(3)$ & $\mathrm{C} 19-\mathrm{C} 20-\mathrm{C} 15-\mathrm{C} 16$ & $-1.0(3)$ \\
\hline $\mathrm{Mo} 02-\mathrm{O} 5-\mathrm{C} 21-\mathrm{C} 22$ & $-152.48(18)$ & $\mathrm{C} 19-\mathrm{C} 18-\mathrm{C} 17-\mathrm{C} 16$ & $-0.5(4)$ \\
\hline $\mathrm{Mo} 02-\mathrm{N} 4-\mathrm{C} 33-\mathrm{C} 32$ & $174.91(17)$ & $\mathrm{C} 15-\mathrm{C} 20-\mathrm{C} 19-\mathrm{C} 18$ & $0.0(3)$ \\
\hline $\mathrm{Mo} 02-\mathrm{N} 4-\mathrm{C} 33-\mathrm{C} 28$ & $-9.1(2)$ & $\mathrm{C} 15-\mathrm{C} 16-\mathrm{C} 17-\mathrm{C} 18$ & $-0.6(4)$ \\
\hline $\mathrm{Mo} 02-\mathrm{N} 4-\mathrm{C} 34-\mathrm{C} 35$ & $72.40(16)$ & $\mathrm{C} 28-\mathrm{N} 3-\mathrm{C} 27-\mathrm{C} 26$ & $-58.0(2)$ \\
\hline $\mathrm{Mo} 02-\mathrm{O} 6-\mathrm{C} 40-\mathrm{C} 35$ & $37.3(3)$ & $\mathrm{C} 28-\mathrm{C} 33-\mathrm{C} 32-\mathrm{C} 31$ & $-2.3(3)$ \\
\hline $\mathrm{Mo} 02-\mathrm{O} 6-\mathrm{C} 40-\mathrm{C} 39$ & $-143.20(19)$ & $\mathrm{C} 28-\mathrm{C} 29-\mathrm{C} 30-\mathrm{C} 31$ & $-0.9(3)$ \\
\hline $\mathrm{Mo} 02-\mathrm{N} 3-\mathrm{C} 28-\mathrm{C} 33$ & $-6.2(2)$ & $\mathrm{C} 1-\mathrm{C} 2-\mathrm{C} 3-\mathrm{C} 4$ & $0.2(4)$ \\
\hline $\mathrm{Mo} 02-\mathrm{N} 3-\mathrm{C} 28-\mathrm{C} 29$ & $176.63(15)$ & $\mathrm{C} 1-\mathrm{C} 2-\mathrm{C} 7-\mathrm{N} 1$ & $48.1(3)$ \\
\hline $\mathrm{Mo} 02-\mathrm{N} 3-\mathrm{C} 27-\mathrm{C} 26$ & $69.68(18)$ & $\mathrm{C} 34-\mathrm{N} 4-\mathrm{C} 33-\mathrm{C} 32$ & $-59.6(3)$ \\
\hline $\mathrm{O} 5-\mathrm{C} 21-\mathrm{C} 22-\mathrm{C} 23$ & $-178.6(2)$ & $\mathrm{C} 34-\mathrm{N} 4-\mathrm{C} 33-\mathrm{C} 28$ & $116.4(2)$ \\
\hline $\mathrm{O} 1-\mathrm{C} 1-\mathrm{C} 6-\mathrm{C} 5$ & $178.5(2)$ & $\mathrm{C} 34-\mathrm{C} 35-\mathrm{C} 36-\mathrm{C} 37$ & $-178.7(2)$ \\
\hline $\mathrm{N} 2-\mathrm{C} 13-\mathrm{C} 12-\mathrm{C} 11$ & $-173.9(2)$ & $\mathrm{C} 6-\mathrm{C} 5-\mathrm{C} 4-\mathrm{C} 3$ & $0.5(4)$ \\
\hline $\mathrm{N} 2-\mathrm{C} 13-\mathrm{C} 8-\mathrm{N} 1$ & $-10.4(3)$ & $\mathrm{C} 25-\mathrm{C} 26-\mathrm{C} 21-\mathrm{O} 5$ & $178.9(2)$ \\
\hline $\mathrm{N} 2-\mathrm{C} 13-\mathrm{C} 8-\mathrm{C} 9$ & $172.8(2)$ & $\mathrm{C} 25-\mathrm{C} 26-\mathrm{C} 21-\mathrm{C} 22$ & $-0.1(3)$ \\
\hline $\mathrm{N} 4-\mathrm{C} 33-\mathrm{C} 32-\mathrm{C} 31$ & $173.9(2)$ & $\mathrm{C} 25-\mathrm{C} 26-\mathrm{C} 27-\mathrm{N} 3$ & $130.2(2)$ \\
\hline $\mathrm{N} 4-\mathrm{C} 33-\mathrm{C} 28-\mathrm{N} 3$ & $10.4(3)$ & $\mathrm{C} 52-\mathrm{N} 8-\mathrm{C} 50-\mathrm{O} 12$ & $-179.7(3)$ \\
\hline $\mathrm{N} 4-\mathrm{C} 33-\mathrm{C} 28-\mathrm{C} 29$ & $-172.52(19)$ & $\mathrm{C} 3-\mathrm{C} 2-\mathrm{C} 1-\mathrm{O} 1$ & $-178.8(2)$ \\
\hline $\mathrm{O} 2-\mathrm{C} 20-\mathrm{C} 19-\mathrm{C} 18$ & $179.4(2)$ & $\mathrm{C} 3-\mathrm{C} 2-\mathrm{C} 1-\mathrm{C} 6$ & $0.6(3)$ \\
\hline $\mathrm{O} 2-\mathrm{C} 20-\mathrm{C} 15-\mathrm{C} 14$ & $2.2(3)$ & $\mathrm{C} 3-\mathrm{C} 2-\mathrm{C} 7-\mathrm{N} 1$ & $-130.8(2)$ \\
\hline $\mathrm{O} 2-\mathrm{C} 20-\mathrm{C} 15-\mathrm{C} 16$ & $179.62(19)$ & $\mathrm{C} 36-\mathrm{C} 35-\mathrm{C} 34-\mathrm{N} 4$ & $124.9(2)$ \\
\hline $\mathrm{O} 6-\mathrm{C} 40-\mathrm{C} 35-\mathrm{C} 34$ & $-2.1(3)$ & $\mathrm{C} 43-\mathrm{N} 5-\mathrm{C} 41-\mathrm{O} 9$ & $-180.0(3)$ \\
\hline $\mathrm{O} 6-\mathrm{C} 40-\mathrm{C} 35-\mathrm{C} 36$ & $-179.60(18)$ & $\mathrm{C} 14-\mathrm{N} 2-\mathrm{C} 13-\mathrm{C} 12$ & $61.0(3)$ \\
\hline $\mathrm{O} 6-\mathrm{C} 40-\mathrm{C} 39-\mathrm{C} 38$ & $-179.3(2)$ & $\mathrm{C} 14-\mathrm{N} 2-\mathrm{C} 13-\mathrm{C} 8$ & $-115.1(2)$ \\
\hline $\mathrm{N} 1-\mathrm{C} 8-\mathrm{C} 9-\mathrm{C} 10$ & $-175.1(2)$ & $\mathrm{C} 14-\mathrm{C} 15-\mathrm{C} 16-\mathrm{C} 17$ & $178.7(2)$ \\
\hline $\mathrm{C} 40-\mathrm{C} 35-\mathrm{C} 34-\mathrm{N} 4$ & $-52.4(2)$ & $\mathrm{C} 7-\mathrm{N} 1-\mathrm{C} 8-\mathrm{C} 13$ & $-119.7(2)$ \\
\hline $\mathrm{C} 40-\mathrm{C} 35-\mathrm{C} 36-\mathrm{C} 37$ & $-1.3(3)$ & $\mathrm{C} 7-\mathrm{N} 1-\mathrm{C} 8-\mathrm{C} 9$ & $57.1(2)$ \\
\hline $\mathrm{C} 40-\mathrm{C} 39-\mathrm{C} 38-\mathrm{C} 37$ & $-0.9(4)$ & $\mathrm{C} 7-\mathrm{C} 2-\mathrm{C} 1-\mathrm{O} 1$ & $2.3(3)$ \\
\hline $\mathrm{C} 33-\mathrm{N} 4-\mathrm{C} 34-\mathrm{C} 35$ & $-54.5(2)$ & $\mathrm{C} 7-\mathrm{C} 2-\mathrm{C} 1-\mathrm{C} 6$ & $-178.3(2)$ \\
\hline $\mathrm{C} 33-\mathrm{C} 32-\mathrm{C} 31-\mathrm{C} 30$ & $-0.5(3)$ & $\mathrm{C} 7-\mathrm{C} 2-\mathrm{C} 3-\mathrm{C} 4$ & $179.1(2)$ \\
\hline $\mathrm{C} 13-\mathrm{N} 2-\mathrm{C} 14-\mathrm{C} 15$ & $56.2(2)$ & $\mathrm{C} 27-\mathrm{N} 3-\mathrm{C} 28-\mathrm{C} 33$ & $119.63(19)$ \\
\hline $\mathrm{C} 13-\mathrm{C} 12-\mathrm{C} 11-\mathrm{C} 10$ & $0.3(3)$ & $\mathrm{C} 27-\mathrm{N} 3-\mathrm{C} 28-\mathrm{C} 29$ & $-57.5(3)$ \\
\hline $\mathrm{C} 13-\mathrm{C} 8-\mathrm{C} 9-\mathrm{C} 10$ & $1.6(3)$ & $\mathrm{C} 27-\mathrm{C} 26-\mathrm{C} 21-\mathrm{O} 5$ & $-2.5(3)$ \\
\hline $\mathrm{C} 2-\mathrm{C} 1-\mathrm{C} 6-\mathrm{C} 5$ & $-0.8(4)$ & $\mathrm{C} 27-\mathrm{C} 26-\mathrm{C} 21-\mathrm{C} 22$ & $178.4(2)$ \\
\hline $\mathrm{C} 2-\mathrm{C} 3-\mathrm{C} 4-\mathrm{C} 5$ & $-0.8(4)$ & $\mathrm{C} 27-\mathrm{C} 26-\mathrm{C} 25-\mathrm{C} 24$ & $-179.3(2)$ \\
\hline $\mathrm{C} 20-\mathrm{C} 19-\mathrm{C} 18-\mathrm{C} 17$ & $0.8(4)$ & $\mathrm{C} 22-\mathrm{C} 23-\mathrm{C} 24-\mathrm{C} 25$ & $-0.8(4)$ \\
\hline $\mathrm{C} 20-\mathrm{C} 15-\mathrm{C} 14-\mathrm{N} 2$ & $53.3(2)$ & $\mathrm{C} 16-\mathrm{C} 15-\mathrm{C} 14-\mathrm{N} 2$ & $-124.0(2)$ \\
\hline
\end{tabular}




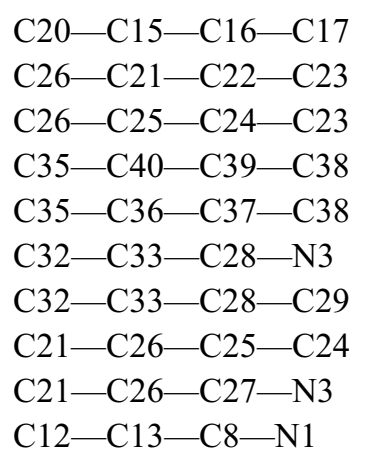

$\mathrm{C} 20-\mathrm{C} 15-\mathrm{C} 16-\mathrm{C} 17$

$\mathrm{C} 26-\mathrm{C} 21-\mathrm{C} 22-\mathrm{C} 23$

$\mathrm{C} 26-\mathrm{C} 25-\mathrm{C} 24-\mathrm{C} 23$

$\mathrm{C} 35-\mathrm{C} 40-\mathrm{C} 39-\mathrm{C} 38$

$\mathrm{C} 35-\mathrm{C} 36-\mathrm{C} 37-\mathrm{C} 38$

$\mathrm{C} 32-\mathrm{C} 33-\mathrm{C} 28-\mathrm{N} 3$

$\mathrm{C} 32-\mathrm{C} 33-\mathrm{C} 28-\mathrm{C} 29$

$\mathrm{C} 21-\mathrm{C} 26-\mathrm{C} 27-\mathrm{N} 3$

$\mathrm{C} 12-\mathrm{C} 13-\mathrm{C} 8-\mathrm{N} 1$
$1.3(3)$

$0.5(4)$

$1.1(4)$

$0.2(3)$

$0.6(4)$

$-173.44(19)$

$3.6(3)$

-0.7 (4)

$-48.4(3)$

$173.4(2)$

$$
\begin{aligned}
& \mathrm{C} 4-\mathrm{C} 5-\mathrm{C} 6-\mathrm{C} 1 \\
& \mathrm{C} 46-\mathrm{N} 6-\mathrm{C} 44-\mathrm{O} 10 \\
& \mathrm{C} 24-\mathrm{C} 23-\mathrm{C} 22-\mathrm{C} 21 \\
& \mathrm{C} 42-\mathrm{N} 5-\mathrm{C} 41-\mathrm{O} 9 \\
& \mathrm{C} 51-\mathrm{N} 8-\mathrm{C} 50-\mathrm{O} 12 \\
& \mathrm{C} 48-\mathrm{N} 7-\mathrm{C} 47-\mathrm{O} 11 \\
& \mathrm{C} 30-\mathrm{C} 29-\mathrm{C} 28-\mathrm{N} 3 \\
& \mathrm{C} 30-\mathrm{C} 29-\mathrm{C} 28-\mathrm{C} 33 \\
& \mathrm{C} 49-\mathrm{N} 7-\mathrm{C} 47-\mathrm{O} 11 \\
& \mathrm{C} 45-\mathrm{N} 6-\mathrm{C} 44-\mathrm{O} 10
\end{aligned}
$$

$0.3(4)$

$0.9(4)$

\begin{tabular}{|c|c|c|c|c|}
\hline$D-\mathrm{H} \cdots A$ & $D-\mathrm{H}$ & $\mathrm{H} \cdots A$ & $D \cdots A$ & $D-\mathrm{H} \cdots A$ \\
\hline $\mathrm{N} 2-\mathrm{H} 008 \cdots \mathrm{O} 11$ & 1.00 & 2.03 & $2.958(2)$ & 154 \\
\hline $\mathrm{N} 4-\mathrm{H} 009 \cdots \mathrm{O} 10$ & 1.00 & 1.99 & $2.924(3)$ & 154 \\
\hline $\mathrm{N} 1-\mathrm{H} 00 D^{\cdots} \mathrm{O} 12$ & $0.85(3)$ & $2.15(3)$ & $2.949(3)$ & $157(2)$ \\
\hline $\mathrm{N} 3-\mathrm{H} 00 E^{\cdots} \mathrm{O} 9$ & $0.79(3)$ & $2.16(3)$ & 2.885 & $154(3)$ \\
\hline
\end{tabular}

$-0.1(4)$

$1.1(4)$

$-0.8(4)$

$-177.0(3)$

174.9 (2)

$-2.0(3)$

-0.7 (4)

$177.3(3)$

Hydrogen-bond geometry $\left(A,{ }^{\circ}\right)$ 